\title{
Diffusion and Superdiffusion from Hydrodynamic Projections
}

\author{
Benjamin Doyon ${ }^{1}$
}

Received: 13 May 2021 / Accepted: 2 December 2021 / Published online: 13 January 2022

(c) The Author(s) 2022

\begin{abstract}
Hydrodynamic projections, the projection onto conserved charges representing ballistic propagation of fluid waves, give exact transport results in many-body systems, such as the exact Drude weights. Focussing one one-dimensional systems, I show that this principle can be extended beyond the Euler scale, in particular to the diffusive and superdiffusive scales. By hydrodynamic reduction, Hilbert spaces of observables are constructed that generalise the standard space of conserved densities and describe the finer scales of hydrodynamics. The Green-Kubo formula for the Onsager matrix has a natural expression within the diffusive space. This space is associated with quadratically extensive charges, and projections onto any such charge give generic lower bounds for diffusion. In particular, bilinear expressions in linearly extensive charges lead to explicit diffusion lower bounds calculable from the thermodynamics, and applicable for instance to generic momentum-conserving one-dimensional systems. Bilinear charges are interpreted as covariant derivatives on the manifold of maximal entropy states, and represent the contribution to diffusion from scattering of ballistic waves. An analysis of fractionally extensive charges, combined with clustering properties from the superdiffusion phenomenology, gives lower bounds for superdiffusion exponents. These bounds reproduce the predictions of nonlinear fluctuating hydrodynamics, including the Kardar-Parisi-Zhang exponent $2 / 3$ for sound-like modes, the Levy-distribution exponent $3 / 5$ for heat-like modes, and the full Fibonacci sequence.
\end{abstract}

\section{Introduction}

Finding organising principles for emergent many-body behaviours is one of the most important tasks of theoretical physics. Thermodynamics and hydrodynamics offer powerful frameworks, based on the postulates that states and dynamics emerging at large scales are described by few, long-lived degrees of freedom [1]. However, characterising these degrees of freedom and quantifying their effects are difficult problems. This is especially true beyond the Euler scale, where one would like to study the Onsager matrix, representing diffusion, and other objects in a model-independent way. In one dimension, a particularly rich phenomenol-

Communicated by Hal Tasaki.

$凶$ Benjamin Doyon

benjamin.doyon@kcl.ac.uk

1 Department of Mathematics, King's College London, Strand, London WC2R 2LS, UK 
ogy of diffusion and superdiffusion is observed. Nonlinear fluctuating hydrodynamics (NFH) [2] offers flexible tools and has been very successful especially for classical, conventional fluids but also beyond, see e.g. [3-9]. Less phenomenological methods based on microscopics include bound on diffusion from conserved quantities [10], and in integrable systems, exact results from generalised hydrodynamics $[11,12]$ and Bethe-ansatz techniques, which have provided much insight [9,13-20]. But despite this progress, a framework for establishing general and rigorous results is still largely lacking.

In this paper, focussing on one dimension of space, I propose such a framework. I show that observables of quantum and classical many-body systems can be organised into equivalence classes with Hilbert space structures, which naturally extract their contributions at finer and finer scales of hydrodynamics. The construction is based on the available one-parameter groups of transformations, such as space and time translations, and clustering properties of local and quasi-local observables, and is similar to the Gelfand-Naimark-Segal construction in $C^{*}$ algebras. The Drude weight, Onsager matrix, hydrodynamic equations and hydrodynamic entropy production are naturally expressed using the inner products on Hilbert spaces associated to the ballistic and diffusive scales. In particular, the diffusive Hilbert space $\mathscr{H}_{\text {dif }}$ gives rise to a formally exact projection formula for the Onsager matrix, similar to the standard hydrodynamic projection $[1,21]$ for the Drude weights. Superdiffusive scales correspond to a family of Hilbert spaces parameterised by the superdiffusive exponent.

I explain how these Hilbert spaces can be related to spaces of conserved charges with various extensivity properties. The linearly extensive charges, studied in greater detail in $[22,23]$, represent the ballistic scale. These are simply related to the standard conserved quantities of many-body systems, such as the total energy and momentum, and are known to bound the Drude weights [24-28]. I show, likewise, that the quadratically extensive charges give rise to generic lower bounds on the Onsager matrix coefficients, generalising Prosen's lower bound [10]. Further, fractionally extensive charges give generic lower bounds for superdiffusion exponents.

Bilinear expressions in conserved quantities provide examples of quadratically and fractionally extensive charges. Analysing these, I obtain a number of results providing explicit lower bounds for diffusion and superdiffusion, which are calculable purely from the thermodynamic averages of conserved densities and currents. The strength of the result depends on the assumed space-time clustering properties of three-point functions in the state of interest.

Under Lieb-Robinson-type clustering, the diagonal Onsager matrix element for the current $j_{k}$ is strictly positive, and may be infinite, if $\left\langle Q_{i} Q_{j} j_{k}\right\rangle^{\mathrm{c}}-\sum_{m n}\left\langle Q_{i} Q_{j} q_{m}\right\rangle^{\mathrm{c}} \mathrm{C}^{m n}\left\langle Q_{m} j_{k}\right\rangle^{\mathrm{c}}$ does not vanish for some $i, j$ ( $q_{i}$ are conserved densities, $Q_{i}$ their total space integral, $\mathrm{C}_{i j}$ is the static covariance matrix, $C^{i j}$ is its inverse, and $\langle\cdots\rangle^{\mathrm{c}}$ are connected correlation functions in some maximal entropy state). In particular, consider a conventional, isentropic, one-dimensional Galilean gas, where the number of particles and their total momentum are the only relevant conserved charges. Its Navier-Stokes equation for the velocity field $v$ is $\partial_{t} v+v \partial_{x} v=\rho^{-1}\left(-\partial_{x} P+\zeta \partial_{x}^{2} v\right)$, where $\rho$ is the gas density, $P$ the pressure and $\zeta$ the bulk viscosity. Then, by hydrodynamic projections, we find that the bulk viscosity is bounded as

$$
\zeta \geq \frac{\rho \chi}{2 v_{\mathrm{LR}}}\left(\partial_{\rho} v_{\mathrm{S}}\right)^{2}
$$

where $\chi=T \partial \rho / \partial \mu$ is the susceptibility ( $T$ the temperature, $\mu$ the chemical potential), $v_{\mathrm{S}}=\sqrt{\mathrm{d} P / \mathrm{d} \rho}$ is the speed of sound and $v_{\mathrm{LR}}$ plays the role of the Lieb-Robinson velocity; see Eq. (105).

Result (1) is shown from general clustering assumptions, and, I expect, can be made rigorous in a large family of models. However, it is known from NFH that, in many cases, $\zeta$ is in fact 
infinite. A formal linear response calculation of Euler-scale three-point functions indicates that clustering is in fact controlled by hydrodynamic velocities. This gives stronger lower bounds. In particular, it reproduces the result from NFH that the diagonal Onsager matrix elements (and in particular $\zeta$ ) must be infinite whenever the NFH three-point couplings are nonzero. Further assuming finer clustering properties set by power laws around ballistic trajectories, I show that the superdiffusion exponent is bounded from below as $\alpha \geq 2 / 3$ if the fully diagonal three-point coupling is nonzero and the scaling function has finite variance. This lower bound is the exponent of the Kardar-Parisi-Zhang universality class that is expected by NFH in this case. I obtain other lower bounds from partially diagonal three-point couplings and scaling functions with fat tails, which, if saturated, reproduce exponents for the heat mode (3/5) and for the Fibonacci sequence [5-7] predicted by NFH and mode coupling theory $[2,5]$. Thus, various aspects of superdiffusion up to now only accessible via NFH, are seen as consequences of Hilbert space structures along with assumptions of clustering, without the need for the hydrodynamic equation or the addition of phenomenological noise.

The space of linearly extensive charges is the tangent space to the manifold of maximal entropy states. I explain how covariant derivatives are obtained from bilinear charges. The Hilbert space they span, the "wave scattering space" $\mathscr{H}_{\text {scat }} \subset \mathscr{H}_{\text {dif }}$, has the physical interpretation as that of two-body scattering states of ballistic waves. In particular, in this language, states formed of co-propagating (same-velocity) ballistic waves are at the source of superdiffusion.

Projection onto $\mathscr{H}_{\text {scat }}$ reproduces a formula for the Onsager matrix conjectured recently [29] from ideas based on NFH. As anticipated in this work, this formula is therefore here shown to provide a lower bound for the Onsager matrix. It is observed in [29] that this formula agrees, when specialised to the hydrodynamics of integrable systems $[11,12]$, with the exact Onsager matrix. The latter was obtained by a form factor expansion in [13,14], and its diagonal part was derived from a linear response mechanism in [15]. Thus, the projection onto $\mathcal{H}_{\text {scat }}$ saturates the Onsager matrix in integrable systems. From this perspective, the present results and techniques put in a precise framework some of the ideas in [13-15,29].

Finally, interpreting the diffusive and wave-scattering Hilbert spaces, I propose a notion of many-body integrability that parallels the Liouville condition that the number of conservation laws must agree with that of degrees of freedom.

The paper is organised as follows. In Sect. 3 the hydrodynamic spaces are introduced, and their relation with the Drude weight and the Onsager matrix are explained. In Sect. 4, extensive charges are defined and their consequences on generic lower bounds for diffusion and superdiffusion are proven. In Sect. 5, bilinear charges are introduced, and the explicit lower bounds discussed above are derived. In Sect. 6, the relation with the hydrodynamic equation, the geometric interpretation of the ballistic and wave-scattering spaces, and the proposal for many-body integrability are discussed. Concluding remarks are made in Sect. 7. All calculations are done in a general framework for one-dimensional many-body systems under natural assumptions, and we expect the results to be applicable to one-dimensional models such as chains and gases of particles, integrable or not. Mathematically rigorous lower bounds in various families of models will be obtained in future works.

\section{Superdiffusion Bounds}

Fourier's law and diffusive hydrodynamics are irreversible large-scale equations. Yet they are expected to emerge in many reasonable isolated, extended system, whose microscopic 
dynamics is reversible. Connecting irreversible characteristics to microscopic quantities without phenomenological inputs such as noise or collision assumptions - the essence of Hilbert's sixth problem - is one of the most important challenges of theoretical and mathematical physics. In particular, given the ubiquity of diffusive effects, an important question is as to the existence of universal, model-independent principles that would establish its presence from simpler conditions. What basic properties of the reversible dynamics and of the state guarantee the emergence of diffusion?

In this section, I overview some of the main ideas that emerge from the organisation principles based on Hilbert spaces, developed in the sections that follow. I explain how to bound the strength of diffusion and superdiffusion-dynamical quantities-by non-dynamical, equaltime correlation functions - static quantities-, in a universal, largely model-independent way. Non-dynamical correlation functions are more easily accessible than dynamical quantities, and thus, I hope, this is a small step towards extracting irreversible characteristics from microscopic quantities, using a limited amount phenomenological input. The sections that follow put this into a formal context, taking into account the full space of hydrodynamic modes. However, for illustration, in this section the argument is presented in its simplest form, for a system of interacting classical particles under simple assumptions, concentrating on a single fluid mode.

I explain how, in one and two dimensions of space, it is possible to establish that hydrodynamic (super)diffusion must be present whenever certain susceptibilities are nonzero. Although the rest of the paper concentrates on one-dimensional systems, it is useful and not more costly here to address higher-dimensionality. Explicit bounds are obtained on the Onsager matrix that represents diffusion, and on the dynamical exponents controlling superdiffusion. I find, generically, the Kardar-Parisi-Zhang dynamical exponent $3 / 2$ in one dimension, and logarithmic superdiffusion in two dimensions, in agreement with previous studies. These results are based on a certain physically plausible assumption on 3-point dynamical correlation functions, pictorially represented in Fig. 1, which is difficult to prove rigorously with current techniques. In the sections that follow, it is also shown that under weaker assumptions, most likely easier to prove, the weaker result of nonzero diffusion (positive Onsager matrix) follows, in particular leading to (1).

\subsection{Diffusion and Superdiffusion in One and Two Dimensions}

Because of the kinematic constraints of low-dimensionality, diffusive effects have been observed to be anomalous in many one- and two-dimensional systems. On the one hand, one way to express this is to discuss correlation functions of local observables. Consider an interacting system of particles, with $r_{i}(t)$ the positions of the particles at time $t$, and its fluid density $n(\mathbf{x}, t)=\sum_{i} \delta\left(\mathbf{x}_{i}-\mathbf{r}_{i}(t)\right)$. Consider the connected correlation functions at two space-time points in a thermodynamic equilibrium state, $\langle n(\mathbf{x}, t) n(0,0)\rangle^{\mathrm{c}}=$ $\langle n(\mathbf{x}, t) n(0,0)\rangle-\langle n(\mathbf{x}, t)\rangle\langle n(0,0)\rangle$. By linear response, such correlation functions obey the hydrodynamic equation. Diffusive effects give rise to the spreading of the correlation around the ballistic trajectories of normal modes, such as the sound mode. Around a ballistic trajectory of velocity $\mathbf{v}$, at large time $t \rightarrow \infty$, normal diffusion, from hydrodynamic equations such as Fourier's law, imply

$$
\langle n(\vec{x}, t) n(0,0)\rangle^{\mathrm{c}} \sim \frac{\chi}{g(t)^{d}} f\left(\frac{\vec{x}-\vec{v} t}{g(t)}\right),
$$


where $d$ is the dimension of space and $\chi$ is the static susceptibility $\left(\int \mathrm{d}^{d} u f(\mathbf{u})=1\right)$, with

$$
g(t)=(\lambda t)^{1 / z}
$$

and dynamical exponent $z=2$, and with the Gaussian form $f(\vec{u})=(2 \pi)^{-d / 2} e^{-|\vec{u}|^{2} / 2}$. The constant $\lambda>0$ is the diffusion constant. By contrast, one-dimensional classical systems such as chains of anharmonically coupled oscillators display superdiffusion. In these cases, the spreading of density correlation functions around the ballistic trajectory respects (2), but with the dynamical exponent $z=3 / 2$, characteristic of the Kardar-Parisi-Zhang (KPZ) universality class. In fact, by the powerful theory of nonlinear fluctuating hydrodynamics (NFH) [2], the shape of the spreading $f(z)$ is a universal correlation function from the KPZ universality class. This function numerically looks like a Gaussian, but with faster-decaying tails.

On the other hand, a practical way to access diffusive effects in classical fluids is to consider the growth of the square-displacement of a tagged particle, say $\left|\Delta \vec{r}_{1}(t)\right|^{2}$ with $\Delta r_{i}(t)=\vec{r}_{i}(t)-\vec{r}_{i}(0)$. The variance with normal diffusive behaviour is

$$
\left\langle\left|\vec{\Delta} r_{1}(t)\right|^{2}\right\rangle^{\mathrm{c}} \sim G(t)^{2}
$$

with

$$
G(t)=(\Lambda t)^{1 / Z}
$$

and again dynamical exponent $Z=2$, where $\Lambda$ is related to the diffusion constant (see below). In two-dimensional fluids such as the hard- or soft-sphere fluids, numerical observations and mode-coupling theory suggest that $G(t)$ receives logarithmic corrections, $G(t)=\sqrt{\Lambda t}(\log t)^{\Gamma}$ where one observes, depending on the model or analysis, $\Gamma=1 / 2$ or $\Gamma=1 / 4[30]$.

The "correlation spread" $g(t)$ and "trajectory spread" $G(t)$ both represent dynamical effects, and are related to each other thanks to the conservation laws. Indeed, consider the local current $\vec{j}(x, t)=\sum_{i} \vec{v}_{i}(t) \delta\left(\vec{x}_{i}-\vec{r}_{i}(t)\right)$, where $\vec{v}_{i}(t)=\mathrm{d} \vec{r}_{i}(t) / \mathrm{d} t$ is the velocity of particle $i$. Then one finds

$$
\left\langle|\vec{J}(t)|^{2}\right\rangle^{\mathrm{c}}=\sum_{i j}\left\langle\vec{\Delta} r_{i}(t) \cdot \vec{\Delta} r_{j}(t)\right\rangle^{\mathrm{c}}
$$

where the space-time integrated current on the system's volume $V$ is $\vec{J}(t)=\int_{0}^{t} \mathrm{~d} s \int_{V} \mathrm{~d}^{d} x \vec{j}(\vec{x}$, $s)$. In the thermodynamic limit, where translation invariance is recovered and assuming that the particles are not correlated, we get $\sum_{i j}\left\langle\vec{\Delta} r_{i}(t) \cdot \vec{\Delta} r_{j}(t)\right\rangle^{\mathrm{c}} \sim V \rho\left\langle\left|\vec{\Delta} r_{1}(t)\right|^{2}\right\rangle^{\mathrm{c}}$ where $\rho=\langle n\rangle$ is the average density, and thus from (4),

$$
t \int_{0}^{t} \mathrm{~d} s \int \mathrm{d}^{d} x\langle\vec{j}(\vec{x}, t) \cdot \vec{j}(0,0)\rangle^{\mathrm{c}} \sim \rho G(t)^{2} .
$$

Using the conservation law $\partial_{t} n+\nabla \cdot \mathbf{j}=0$, and assuming the form (2) where $f(\mathbf{u})$ has finite variance $\Delta f=\int \mathrm{d}^{d} u|\mathbf{u}|^{2} f(\mathbf{u})$, one obtains (see Appendix 1)

$$
G(t)^{2} \sim \frac{\chi \Delta f}{\rho} \operatorname{tg}^{\prime} g .
$$

For the power law behaviours described above, $g(t)=(\lambda t)^{1 / z}$ and $G(t)=(\Lambda t)^{1 / Z}$, this gives $\Lambda=\left(\frac{\chi \Delta f}{z \rho}\right)^{z / 2} \lambda$ and

$$
Z=z
$$


(the diffusive case $z=2$ gives $\Lambda=\frac{d \times \lambda}{2 \rho}$ ). Logarithmic corrections to diffusive behaviours $g(t)=\sqrt{\lambda t}(\log t)^{\gamma}$ and $G(t)=\sqrt{\Lambda t}(\log t)^{\Gamma}$ are related as

$$
\Gamma=\gamma \text {. }
$$

Two comments are in order concerning this simple analysis.

1. In dimensions greater than one, some hydrodynamic modes propagate into shells of codimension 1, not described by the form (2). Further, in general (and generically in one dimension), there may be many ballistic modes with different velocities $\mathbf{v}_{a}$. These have associated partial susceptibilities $\chi_{a}$ (summing to the full susceptibility $\chi=\sum_{a} \chi_{a}$ ), correlation types $g_{a}(t)$ and shapes $f_{a}(\mathbf{u})$. In the above analysis, one has to sum over these modes, keeping the dominant correlation type in the large- $t$ asymptotics. A more accurate treatment makes use of normal modes, as explained in the sections that follow. Here, I keep the simple assumption that (2) is sufficient in order to illustrate the ideas.

2. The assumption that $f(\mathbf{u})$ has finite variance is immediate in the case of diffusion, thanks to the Gaussian distribution. It also holds for KPZ superdiffusion-which we may refer to as "normal superdiffusion". However, there are types of superdiffusion that break it, for instance those related to Levy processes, as for the heat mode in one dimension [2,5]. We will refer to this as "fat superdiffusion". In these cases, relations (9) and (10) are broken. There is currently no known a priori criterium for establishing if the variance of $f$ is finite or not, the cases being studied to date relying on numerical observation or the phenomenological theory of NFH. However, from an analysis of the known examples, in $d=1$, it appears as though there is a special class fat superdiffusion that is relevant. The phenomenology for how to modify (8), and the result, are as follows.

First, results from NFH suggest that, in all fat-superdiffusive cases, the normalisation coefficient in the fat tail of $\langle n(x, t) n(0,0)\rangle^{\mathrm{c}}$ at large $|x|$, grows linearly in time, $\langle n(x, t) n(0,0)\rangle^{\mathrm{c}} \sim c t|x|^{v}$ at large $|x|$ for some $-3<v<-1$. Here the bounds on $v$ come from the constraints of a divergent variance but a finite, time-independent susceptibility. Therefore,

$$
f(u) \sim c^{\prime}|u|^{-(1+z)} \quad(|u| \rightarrow \infty) .
$$

In particular, this means that $v=-(1+z)$, and so $z \in(0,2)$, in agreement with superdiffusion. Second, in (8), the current-current correlation function must be integrated only on the region of space relevant to the fat-superdiffusive mode. This region grows linearly in time, and is bounded by the trajectories, of velocities $v_{1}<v, v_{2}>v$, of the modes that surround the fat-superdiffusive mode, of velocity $v$ (for instance, the heat mode is bounded by the two sound modes in typical momentum-conserving models). From this, one obtains (see Appendix 1)

$$
G(t)^{2} \sim \frac{c\left(a^{2-z}+b^{2-z}\right)}{(2-z) \rho} t^{3-z}
$$

where $a=\left|v_{1}-v\right|, b=\left|v_{2}-v\right|$. Thus one has, instead of (9), the relation

$$
\left.Z=\frac{2}{3-z} \in(2 / 3,2) \quad \text { (fat superdiffusion, } d=1\right) \text {. }
$$

\section{2 (Super)diffusion Bounds from Static Correlations}

The above relations between correlation and trajectory spreads follow in a relatively simple fashion from the conservation laws. The results of this paper amount to a new relation, which 
makes use of the Cauchy-Schwartz inequality and fundamental principles of many-body physics. This new relation is powerful enough to show that, under a certain natural condition, there must be diffusion or superdiffusion in any dimension $d \in[0,2]$. Further, it shows that in $d=1$, we must have $z \leq 3 / 2$ for finite-variance superdiffusion.

This section is an overview of some of the calculations in Sect. 5.4. Sections 5.2 and 5.3 present similar calculations, but based on weaker assumptions, showing only, respecticely, positive diffusion [thus not necessarily superdiffusion, and leading to (1)], and infinite diffusion (thus superdiffusion, but without information about the exponents).

In order to express the result, it is convenient to introduce the ordering $\succeq$ between functions $g(t), h(t)$ which, at large $t$, grow monotonically. For any two such functions, the limit $\lim _{t \rightarrow \infty} g(t) / h(t)$ exists in the extended non-negative real numbers: it is either $\infty$, a finite number, or 0 . We say that $g(t) \succeq h(t)$ if $\lim _{t \rightarrow \infty} g(t) / h(t)>0$

The main result which we overview here is as follows: define the 3-point self-coupling as

$$
C:=\min _{i}\left\{\int \mathrm{d}^{d} x \mathrm{~d}^{d} y\left\langle n(\vec{x}, 0) n(\vec{y}, 0) j^{i}(\vec{O}, 0)\right\rangle^{\mathrm{c}}\right\} \neq 0 .
$$

Then

$$
G(t)^{2} g(t)^{d} \succeq(C t)^{2}
$$

That is, correlations in space, as characterised by $C$, impose a relation between correlation spreading, and trajectory spreading, which are dynamical quantities. This is a type of "fluctuation-dissipation" theorem, involving the third cumulant for the statistical fluctuations of observables.

In particular, assuming the power laws with the relation (9) valid for finite variance, $G(t) \propto t^{1 / z}$ and $g(t) \propto t^{1 / z}$, this gives

$$
z \leq 1+\frac{d}{2}
$$

For $d=1$, we recover the KPZ exponent as an upper bound, $z \leq 3 / 2$, and the condition of nonzero 3-point self coupling agrees with that found from NFH; and thus we derive the presence of superdiffusion without using noisy dynamics. For $d=2$ we find normal diffusion as an upper bound, $z \leq 2$. Assuming logarithmic corrections to normal diffusion and the relation (10), $g(t) \propto \sqrt{t}(\log t)^{\gamma}$ and $G(t) \propto \sqrt{t}(\log t)^{\gamma}$, the relation can only be satisfied in $d \geq 2$, and gives

$$
\gamma \geq 0 \text {. }
$$

That is, logarithmic corrections may be superdiffusive, but may not be subdiffusive, in agreement with previous studies. In higher dimensions $d>2$, there may be subdiffusion.

For $d=1$ and fat superdiffusion (infinite variance), using (13) we obtain the bound

$$
z \leq \frac{1+\sqrt{5}}{2}
$$

This is a new result. We observe that this value is the limit of the Fibonacci sequence obtained for superdiffusive modes associated to Levy processes in [5]. Levy processes have infinite variance, so these are indeed fat-superdiffusive modes. These modes have zero 3-point self coupling in the Fibonacci sequence theory (this includes the heat mode in generic anharmonic chains, which has $z=5 / 3>\frac{1+\sqrt{5}}{2}$ ), so are not constrained by (18); however the limit tends, formally, to a mode with nonzero 3-point self coupling, and thus it makes sense that the limit be constrained by (18). 


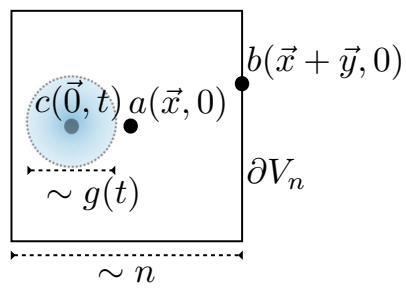

Fig. 1 Pictorial representation of the condition on three-point correlation function. This is an extension to three-point functions of the correlation spreading form (2) for two-point functions, involving the spreading $g(t)$. We see that if $n \gg g(t)$, it is not possible for both $a(\mathbf{x}, 0)$ and $b(\mathbf{x}+\mathbf{y}, 0)$ to be simultaneously within the blue shaded region, and thus by clustering the connected correlation function vanishes

The derivation presented below takes without loss of generality zero expectation values of densities and currents (which can be achieved by appropriate shifts), and for simplicity assumes that the fluid mode's velocity is zero $\mathbf{v}=\mathbf{0}$. It also relies on two assumptions: (a) That 4-point functions in space cluster fast enough; this is expected to be easy to prove in various models and states with current methods. (b) That dynamical 3-point functions cluster, in accordance with the correlation spread of (2):

$$
\langle a(\vec{x}, 0) b(\vec{x}+\vec{y}, 0) c(\vec{O}, t)\rangle^{\mathrm{c}} \rightarrow 0 \quad(|\vec{x}| \gg g(t) \text { or }|\vec{x}+\vec{y}| \gg g(t))
$$

See Fig. 1. This (or the required precise form) is much harder to prove, although with the less restrictive choice $z=1$ this is expected to follow from the Lieb-Robinson bound [31] (to my knowledge the proof has not been worked out yet).

In order to derive the main result (15), consider the observables

$$
Q_{n}(\mathbf{x})=\int_{V_{n}} \mathrm{~d}^{d} y n(\mathbf{x}+\mathbf{y}, 0) n(\mathbf{x}, 0)
$$

where $V_{n}=[-n / 2, n / 2]^{d}$ is the $d$-dimensional cube of linear size $n$. These are examples of bilinear charges, see Sect. 5.1. Then, by using clustering of the state $\langle\cdots\rangle$, we obtain

$$
\int \mathrm{d}^{d} x\left\langle Q_{n}(\mathbf{x}) Q_{n}(\mathbf{0})\right\rangle^{\mathrm{c}} \sim 2 n^{d}\left[\int \mathrm{d}^{d} x\langle n(\mathbf{x}, 0) n(\mathbf{0}, 0)\rangle^{\mathrm{c}}\right]^{2}=2 n^{d} \chi^{2}
$$

where on the left-hand side, $\left\langle Q_{n}(\mathbf{x}) Q_{n}(\mathbf{0})\right\rangle^{\mathrm{c}}=\left\langle Q_{n}(\mathbf{x}) Q_{n}(\mathbf{0})\right\rangle-\left\langle Q_{n}(\mathbf{x})\right\rangle\left\langle Q_{n}(\mathbf{0})\right\rangle$. This is obtained by using clustering of the 4-point function, which implies that the leading large- $n$ asymptotics is given by the two Wick contractions that are not subtracted on the left-hand side. On the other hand, we may evaluate

$$
\int \mathrm{d}^{d} x\left\langle Q_{n}(\mathbf{x}) \partial_{t} \mathbf{j}(\mathbf{0}, t)\right\rangle^{\mathrm{c}}=-\int \mathrm{d}^{d} x\left\langle\partial_{t} Q_{n}(\mathbf{x}) \mathbf{j}(\mathbf{0}, t)\right\rangle^{\mathrm{c}}
$$

by using the conservation laws:

$$
=\int \mathrm{d}^{d} x \int_{\partial V_{n}} \mathrm{~d}^{d-1} y \cdot\left(\langle\mathbf{j}(\mathbf{x}+\mathbf{y}) n(\mathbf{x}, 0) \mathbf{j}(\mathbf{0}, t)\rangle^{\mathrm{c}}+\langle n(\mathbf{x}+\mathbf{y}) \mathbf{j}(\mathbf{x}, 0) \mathbf{j}(\mathbf{0}, t)\rangle^{\mathrm{c}}\right) .
$$

Using three-point clustering property (19), we conclude that $\int \mathrm{d}^{d} x\left\langle Q_{n}(x) \partial_{t} j(0, t)\right\rangle^{\mathrm{c}}=0$ for all $t$ such that $g(t) \ll n$ (see Fig. 1). Therefore, we may average over a time period of extent $0<t_{n} \ll g^{-1}(n)$, 


$$
\int \mathrm{d}^{d} x\left\langle Q_{n}(\mathbf{x}) \mathbf{j}(\mathbf{0}, 0)\right\rangle^{\mathrm{c}}=\frac{1}{t_{n}} \int_{-t_{n} / 2}^{t_{n} / 2} \mathrm{~d} s \int \mathrm{d}^{d} x\left\langle Q_{n}(\mathbf{x}) \mathbf{j}(\mathbf{0}, s)\right\rangle^{\mathrm{c}} .
$$

Now consider $\int \mathrm{d}^{d} x\left\langle a^{\dagger}(\mathbf{x}) b(0)\right\rangle^{\mathrm{c}}$ as a function of the observables $a, b$. It is a sesquilinear form $(a, b)$, and non-negative, as $(a, a)=\lim _{n \rightarrow \infty} n^{-d}\left\langle A_{n}^{\dagger} A_{n}\right\rangle^{\mathrm{c}}$ where $A_{n}=\int_{V_{n}} \mathrm{~d}^{d} x a(x)$. This is the basis for the construction of the "ballistic hydrodynamic space", where spatial coordinates are integrated over, see Sect. 3 and especially Sect. 3.2. Therefore, we write (assuming the conserved densities and currents to be real)

$$
\int \mathrm{d}^{d} x\left\langle Q_{n}(\mathbf{x}) \mathbf{j}(\mathbf{0}, 0)\right\rangle^{\mathrm{c}}=t_{n}^{-1}\left(Q_{n}, \mathbf{j}_{n}\right)
$$

where $j_{n}=\int_{-t_{n} / 2}^{t_{n} / 2} j(0, s)$. By the Cauchy-Schwartz inequality, this is bounded as

$$
\left|\int \mathrm{d}^{d} x\left\langle Q_{n}(\mathbf{x}) j^{i}(0,0)\right\rangle^{\mathrm{c}}\right|^{2} \leq\left(Q_{n}, Q_{n}\right) t_{n}^{-2}\left(j_{n}^{i}, j_{n}^{i}\right)
$$

and thus

$$
C^{2} \leq\left(Q_{n}, Q_{n}\right) t_{n}^{-2}\left(\mathbf{j}_{n} \cdot \mathbf{j}_{n}\right)
$$

On the right-hand side, $\left(\mathbf{j}_{n} \cdot, \mathbf{j}_{n}\right)$ is (simply related to) the inner product at the basis of the "diffusive hydrodynamic space", where time has been integrated over, see again Sect. 3.2. The inequality obtained is a special case of the general inequalities found from an analysis of the hydrodynamic spaces, see Sect. 4; in particular $Q_{n}$ gives rise to a "quadratically extensive charge".

By time-translation invariance and (7), we find $\left(\mathbf{j}_{n} \cdot, \mathbf{j}_{n}\right) \sim t_{n} \int_{0}^{t_{n}} \int \mathrm{d}^{d} x\langle\mathbf{j}(\mathbf{x}, t) \cdot \mathbf{j}(0,0)\rangle^{\mathrm{c}} \sim$ $\rho G\left(t_{n}\right)^{2}$ and using (21), we obtain $\left(Q_{n}, Q_{n}\right)=\int \mathrm{d}^{d} x\left\langle Q_{n}(x) Q_{n}(0)\right\rangle^{\mathrm{c}} \sim 2 n^{d} \chi^{2}$. Combining with (27),

$$
2 \rho \chi^{2} n^{d} G\left(t_{n}\right)^{2} t_{n}^{-2} \succeq C^{2} .
$$

If $G(t)$ grows slower than, or as fast as, $t$, then the tightest condition is obtained by taking $t_{n} \sim g^{-1}(n)$. Writing $n=g(t)$, and using $\rho, \chi^{2}>0$, this becomes (15).

\section{Hydrodynamic Spaces}

In this section I describe an abstract and general construction which organises observables of many-body systems into equivalence classes representing their contributions at various scales of hydrodynamics. One-dimensional systems are the focus, although the extension to higher dimensions is natural.

The construction does not require any specific underlying many-body system. For generality, we consider a countable-dimensional linear space $\mathcal{V}$ over $\mathbb{C}$, the space of local or quasi-local observables, forming an algebra on which there is an anti-linear involution $a \mapsto a^{\dagger}$, and over which there is a state $\langle\cdots\rangle$, a positive linear functional representing the averages. Out of this, a more general setup is extracted, a Hilbert space $\mathscr{H}$ with a positivedefinite sesquilinear form $\langle\cdot, \cdot\rangle_{\mathcal{H}}$. This second setup is obtained from the first by taking the Cauchy completion of $v$ under the topology induced by a natural inner product constructed from $\langle\cdots\rangle$, as in the Gelfand-Naimark-Segal construction. Examples of inner products are

$$
\langle a, b\rangle_{\mathcal{H}}=\left\langle a^{\dagger} b\right\rangle \text { or }\langle a, b\rangle_{\mathcal{H}}=\frac{1}{2}\left\langle a^{\dagger} b+b a^{\dagger}\right\rangle \equiv\left\langle a^{\dagger} \cdot b\right\rangle
$$


or one may use the Kubo-Mori-Bogoliubov (KMB) inner product, with $a \cdot b=$ $\int_{0}^{1} \mathrm{~d} s \rho^{-s} a \rho^{s} b$ is $\langle\cdots\rangle$ is described by the density matrix $\rho$. Here for simplicity it is assumed that the inner product is not degenerate on $V$.

The viewpoint here is that of the $C^{*}$ algebra formulation of quantum and classical statistical mechanics. In the context of spin-1/2 quantum spin chains in the thermodynamic limit (infinite length), for instance, one has a uniformly hyperfinite $C^{*}$ algebras [32,33]; strong results exist and many of the properties we require are shown rigorously, in particular many of the rigorous results of [23] can be applied. In this context, the local observables $v$ can be chosen as those supported on finite numbers of sites:

$$
V=\operatorname{span}\left\{\prod_{x \in \Lambda} \sigma_{j_{x}}(x): \Lambda \subset \mathbb{Z},|\Lambda|<\infty\right\}
$$

where $\sigma_{j}(x)$ acts nontrivially only on site $x \in \mathbb{Z}$, as the Pauly matrix $\sigma_{j}$ (for $j=1,2,3$ ). In the modern understanding, one might consider extending this space to adjoin the quasi-local observables [34], but these details are not crucial below. There is no need for $V$ to take all elements of the $C^{*}$ algebra (we do not need $V$ to be complete with respect to the $C^{*}$ norm). A state $\langle\cdots\rangle$ could be any Kubo-Martin-Schwinger state based on a local Hamiltonian. Similar considerations hold for classical (non-quantum) models.

Note that the Hilbert space $\mathscr{H}$ is not related to any underlying quantum-mechanical Hilbert space of the many-body model; this abstract construction is valid for classical and quantum models, deterministic or stochastic.

\subsection{Hydrodynamic Reduction}

Suppose that there is some action of a one-parameter group of unitary operators $U_{s}$ on $\mathscr{H}$, with $U_{s} U_{s^{\prime}}=U_{s+s^{\prime}}$. I take $s \in \mathbb{R}$, but the main property for the present discussion is that the group be non-compact; there is no difficulty in adapting to $s \in \mathbb{Z}$. In the continuous notation, I assume that $U_{s}$ is "well behaved", that is, that the resulting inner products are continuous and differentiable. By unitarity, $U_{s}$ preserves the inner product,

$$
\left\langle U_{s} a, U_{s} b\right\rangle_{\mathcal{H}}=\langle a, b\rangle_{\mathcal{H}} .
$$

For instance, $U_{s}$ could be the group of space- or time-translations, or any other symmetry of the underlying model, under which the state is chosen to be invariant. Again, note that this does not require the underlying model to be quantum or even deterministic: $U_{s}$ acts, and is invertible, on the abstract Hilbert space $\mathcal{H}$, and unitarity is the result of an invariance.

The Hilbert space $\mathcal{H}$ and its inner product contain a large amount of information about the microscopic many-body model. At large scales, the number of degrees of freedom is reduced, and a small amount of information is sufficient. We would like to extract these emergent properties. As a guiding principle, emergent properties of many-body systems with respect to the group $U_{s}$ are essentially those obtained by looking at invariants. There are two natural ways of extracting invariants.

First, one may simply restrict to the subspace

$$
\mathcal{Q}=\cap_{s} \operatorname{ker}\left(U_{s}-1\right) .
$$

This is the subspace of all observables which are invariant under all $U_{s}$. This is a closed subspace, hence we can make orthogonal projections. Let $\mathbb{P}$ denote the orthogonal projection onto 2 . I call this process hydrodynamic projection, and denote it by 


$$
\begin{aligned}
& \mathcal{H} \\
& \downarrow \mathbb{P} . \\
& 2
\end{aligned}
$$

The second way goes into more details of the behaviour at large $s$. We instead consider the equivalence classes with respect to $U_{s}$, providing this with a Hilbert space structure by integrating over $s$. As the group is non-compact, convergence needs to be imposed, and for this the kernel needs to be taken away. More precisely, suppose that every element $a$ in some subspace $V^{\prime} \subset \mathcal{H}$ projects onto $\mathcal{Q}$ at infinity fast enough: for every $a, b \in V^{\prime}$, there exists $b>0, c>0$ and $d>1$ such that

$$
\left|\left\langle U_{s} a, b\right\rangle_{\mathscr{H}}-\langle\mathbb{P} a, b\rangle_{\mathscr{H}}\right| \leq c(b+|s|)^{-d} .
$$

The quantity $c$ is the overall normalisation, $b$ is in order to encode uniform boundedness, and $d$ is the decay exponent. The precise power law form (34), with $d>1$, is here chosen as it is one of the weakest decay assumption we may impose, yet being strong enough for our purposes.

Then we can form the hydrodynamic Hilbert space $\mathcal{H}^{\prime}$. We consider the new sesquilinear form $^{1}$

$$
\langle a, b\rangle_{\mathcal{H}^{\prime}}=\int \mathrm{d} s\left\langle(1-\mathbb{P}) U_{s} a, b\right\rangle_{\mathcal{H}}
$$

for all $a, b \in V^{\prime}$. The integral is taken over $\mathbb{R}$, and convergent. This is positive semidefinite, as by the assumption (34), we can write it in an explicitly non-negative way,

$$
\langle a, b\rangle_{\mathcal{H}^{\prime}}=\lim _{n \rightarrow \infty} \frac{1}{2 n} \int_{-n}^{n} \mathrm{~d} s \int_{-n}^{n} \mathrm{~d} t\left\langle(1-\mathbb{P}) U_{s} a,(1-\mathbb{P}) U_{t} b\right\rangle_{\mathcal{H}}
$$

(see e.g. [22, Lem 4.2]). We mod out the associated null space $n^{\prime}$, and Cauchy-complete the resulting space of equivalence classes $V^{\prime} / n^{\prime}$, obtaining a new Hilbert space $\mathcal{H}^{\prime}$. On this space, $\mathcal{Q} \equiv \mathscr{H}^{\prime}\{0\}$ as it is projected out, and $U_{s} \equiv \mathscr{H}^{\prime} 1$, as we integrate over $s$ in (36). I call this process the hydrodynamic reduction of $\mathscr{H}$ with respect to $U=\left\{U_{s}: s \in \mathbb{R}\right\}$ with "nucleus" $V^{\prime}$, and pictorially represent it as

$$
\mathscr{H} \stackrel{U}{\rightarrow} \mathscr{H}^{\prime}
$$

(where I keep the information of the subspace $V^{\prime} \subset \mathcal{H}$ hidden).

Note that if I were averaging over $s$ instead of integrating in (36), then by von Neumann's ergodic theorem [35], this would create the projection $\mathbb{P}$. Here I subtract the projection, and then integrate-looking, so to speak, at the next, nontrivial order.

The condition (34) is assumed to hold on $V^{\prime}$. It might not be satisfied for all elements in $\mathcal{H}$. It will be convenient to define the " $\mathcal{H}^{\prime}$-lower norm" on all of $\mathscr{H}$, which takes values in the extended non-negative real numbers $\hat{\mathbb{R}}=\mathbb{R}_{+} \cup\{\infty\}$, by

$$
\|a\|_{\mathscr{H}^{\prime}}^{-}=\liminf _{n \rightarrow \infty} \frac{1}{\sqrt{2 n}}\left\|\int_{-n}^{n} \mathrm{~d} s(1-\mathbb{P}) U_{s} a\right\|_{\mathscr{H}} \in \hat{\mathbb{R}} \quad(a \in \mathcal{H}) .
$$

${ }^{1}$ Note the equivalent ways of writing:

$$
\left\langle(1-\mathbb{P}) U_{s} a, b\right\rangle_{\mathscr{H}}=\left\langle(1-\mathbb{P}) U_{s} a,(1-\mathbb{P}) b\right\rangle_{\mathscr{H}}=\left\langle U_{s} a, b\right\rangle_{\mathscr{H}}-\langle\mathbb{P} a, \mathbb{P} b\rangle_{\mathscr{H}} .
$$


This agrees with the norm $\|a\|_{\mathscr{H}^{\prime}}=\sqrt{\langle a, a\rangle_{\mathcal{H}^{\prime}}}$ if (34) holds for $\mathfrak{b}=a$. The upper norm could also be considered, but as I will be considering lower bounds, the lower norm is more meaningful.

Remark 3.1 Under certain conditions [23], it is possible to show that $\mathscr{H}^{\prime}$, as a set, is exactly (the completion of) the set of equivalence classes of $V^{\prime}$ under $a \equiv a+q$ for $q \in \mathcal{Q} \cap V^{\prime}$, and $a \equiv U_{s} a$ for $s \in \mathbb{R}$.

Remark 3.2 In quantum spin chains, time translation is a strongly continuous one-parameter group on $\mathcal{H}$, hence continuity and differentiability are guaranteed [32,33]. Further, the space 2 may be a very small space; for instance, in many natural states of quantum spin chains, the only observables that are invariant under space translations are those proportional to the "identity observable" $\mathbf{1}$ (the identity operator on the chain) ${ }^{2}$, so $2=\mathbb{C} \mathbf{1}$.

\subsection{Ballistic and Diffusive Spaces}

I now argue that the processes of hydrodynamic projections and reductions are at the basis of the most fundamental objects of hydrodynamics: the Drude weights and the Onsager matrix, which control the Euler and diffusive scales of the hydrodynamic equations. The general viewpoint adopted above allows me to discuss these objects of hydrodynamics in a way that, I hope, makes clear the parallel between the Euler and diffusive scales, shedding light, at least structurally, on the Onsager matrix. Indeed, it suggests that the Onsager matrix is, in a sense, a susceptibility; in particular, the subtraction of the Drude weight is the subtraction of a "disconnected" component, much like is done by writing the usual susceptibilities as space-integrated cumulants.

In homogeneous many-body systems in one dimension, two natural symmetries that the states and dynamics may have, leading to non-compact unitary groups on $\mathcal{H}$, are translations in space and time, which I will denote $\iota_{x}$ and $\tau_{t}$ respectively. Again, here I use the continuous notation, but all results apply in the discrete case as well (for instance, $x \in \mathbb{Z}$ in a quantum chain). In integrable systems, there is in addition an infinite number of such groups: all the flows associated to the higher Hamiltonians of the integrable hierarchy. For generality, I write $\iota_{x}=\tau_{x}^{(1)}, \tau_{t}=\tau_{t}^{(2)}$ and $\eta_{s}=\tau_{s}^{(3)}$, and assume in total a certain number (which may be infinite) $N \geq 2$ of nontrivial flows, $\tau_{s}^{(\ell)}$ for $\ell \geq 1$ with $\tau_{s}^{(\ell)}=1$ for $\ell>N$, which act on $\mathcal{H}$. For instance, $\eta_{s}=1$ is trivial if the chain is not integrable $(N=2)$. I also assume that all flows commute.

Consider the following sequential construction. We first construct the hydrodynamic projection $\mathcal{H}^{\prime}$ of $\mathcal{H}$ with respect to $\iota$, with some nucleus $V^{\prime} \subset \mathcal{H}$. Typically $V^{\prime}=V$, all local and quasi-local observables; their correlations cluster fast enough in KMS states for instance [36]. On $\mathscr{H}^{\prime}$, space translations are trivial, $\iota_{x} \equiv \mathscr{H}^{\prime} 1$ for all $x$. However, time translations are nontrivial, and now act on $\mathcal{H}^{\prime}$. I still denote by $\tau_{t}$ the resulting action on $\mathcal{H}^{\prime}$. Then, we construct the hydrodynamic projection $\mathscr{H}^{\prime \prime}$ of $\mathscr{H}^{\prime}$ with respect to $\tau$ (with nucleus $\mathcal{V}^{\prime \prime} \subset \mathscr{H}^{\prime}$ ). We continue the process until the last nontrivial flow. Pictorially,

$$
\mathcal{H} \stackrel{\iota}{\rightarrow} \mathcal{H}^{\prime} \stackrel{\tau}{\rightarrow} \mathcal{H}^{\prime \prime} \stackrel{\tau^{(3)}}{\rightarrow} \cdots \stackrel{\tau^{(N)}}{\rightarrow} \mathcal{H}^{(N)} \stackrel{1}{\rightarrow}\{0\}
$$

2 On finite chains, homogeneous sums over the full space are trivially translation invariant; but here the chain is infinite, so these sums are not expected to give elements of $\mathcal{H}$ (and are not part of the underlying $C^{*}$ algebra). 
These may be called the hydrodynamic spaces of 0 th, 1 st, 2 nd, ... order. For instance,

$$
\langle a, b\rangle_{\mathcal{H}^{\prime}}=\int \mathrm{d} x\left\langle\left(1-\mathbb{P}_{\iota}\right) \iota_{x} a, b\right\rangle_{\mathcal{H}}, \quad\langle a, b\rangle_{\mathcal{H}^{\prime \prime}}=\int \mathrm{d} t\left\langle\left(1-\mathbb{P}_{\tau}\right) \tau_{t} a, b\right\rangle_{\mathcal{H}^{\prime}}
$$

Let me also consider the spaces of invariants and associated projections. In fact, as the flows commute, it is more natueral to project onto the invariants of all higher flows. I then simply define

$$
Q^{(\ell)}=\cap_{\ell^{\prime}>\ell} \cap_{s} \operatorname{ker}\left(\tau_{s}^{\left(\ell^{\prime}\right)}-1\right)
$$

(with the notation $\mathcal{Q}^{(0)}=\mathcal{Q}, \mathcal{Q}^{(1)}=\mathcal{Q}^{\prime}$, etc.), and denote in particular $\mathbb{P}_{\iota}: \mathcal{H} \rightarrow \mathcal{Q}$, $\mathbb{P}_{\tau}: \mathscr{H}^{\prime} \rightarrow \mathcal{Q}^{\prime}$ and $\mathbb{P}_{\eta}: \mathscr{H}^{\prime \prime} \rightarrow \mathcal{Q}^{\prime \prime}$. Note that $\mathscr{Q}^{(N)}=\mathscr{H}^{(N)}$, and so, in this notation, if $N=2$ (conventional, non-integrable systems) then $\mathbb{P}_{\eta}=1$ is trivial.

I claim that the sequence of spaces of invariants (42) encode the physics of many-body systems at finer and finer hydrodynamic scales. In particular, I call 2 the thermodynamic space, $\mathcal{Q}^{\prime}$ the ballistic space, and $\mathcal{Q}^{\prime \prime}$ the diffusive space:

$$
\mathcal{Q}=\mathscr{H}_{\mathrm{th}}, \quad 2^{\prime}=\mathscr{H}_{\mathrm{bal}}, \quad Q^{\prime \prime}=\mathscr{H}_{\mathrm{dif}} .
$$

Thus, pictorially,

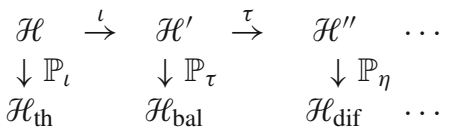

I will use the notation (recall that $\eta$ represent a flow with respect to a higher conserved charge, if any, and $\mathbb{P}_{\eta}=1$ if there aren't any)

$$
\langle a, b\rangle_{\text {bal }}=\left\langle\mathbb{P}_{\tau} a, b\right\rangle_{\mathcal{H}^{\prime}}, \quad\langle a, b\rangle_{\mathrm{dif}}=\left\langle\mathbb{P}_{\eta} a, b\right\rangle_{\mathcal{H}^{\prime \prime}}
$$

In Sect. 3.3, I justify this claim by connecting with the Drude weights and Onsager matrix, and in Sect. 3.4, by making the connection with the hydrodynamic equations.

As is discussed below, $\mathbb{P}_{\iota}$ is just the subtraction of expectation values-this is a trivial part of the construction, which I discuss mainly to establish the structural symmetry. In general $\mathcal{H}_{\text {bal }}$ contains the extensive conserved charges, and so this part of the construction is nontrivial. Finally, if there are no higher flows, for instance in chaotic systems, then $\mathbb{P}_{\eta}=1$ and $\mathcal{H}_{\text {dif }}=\mathscr{H}^{\prime \prime}$. In this case, the diffusive space is formed of all remaining observables after the $\tau$ hydrodynamic reduction (where there is no space-time translation left). We will see below that, by contrast, if $\eta$ is nontrivial, generic elements of $\mathscr{H}^{\prime \prime}$ are not in $\mathscr{H}_{\text {dif }}$, but the currents of conserved densities are; this is relevant to integrable systems.

For simplicity I will keep the same notation of lower-case latin letters $a, b, \ldots$ for elements of $\mathcal{V}, \mathcal{H}, \mathscr{H}^{\prime}, \mathscr{H}^{\prime \prime}$. I use the notation $\equiv \mathscr{H}^{\prime}, \equiv \mathscr{H}^{\prime \prime}$ for equivalences in the indicated spaces, and $[\cdot]_{\mathcal{H}^{\prime}},[\cdot]_{\mathscr{H}^{\prime \prime}}$ for the corresponding equivalence classes, when it is necessary for clarity.

Remark 3.3 The passage from $\tau_{t}$ acting on $\mathscr{H}$ to $\tau_{t}$ acting on $\mathscr{H}^{\prime}$ is nontrival. However, under general conditions (see [22, Thm 6.3] and [23]), including the condition that $V^{\prime}$ be in some sense large enough, $\tau_{t}$ is indeed on $\mathcal{H}^{\prime}$ a well-behaved unitary operator for all $t \in \mathbb{R}$, forming a one-parameter group. For instance, all conditions are satisfied in quantum spin chains with finite local spaces, where we may take $V^{\prime}=V$. 


\subsection{Drude Weights and Onsager Matrix}

Consider the algebra $v$ of observables. First, as mentioned in Remark 3.2, in most cases, the only translation-invariant local observables are those proportional to the identity, $\mathscr{H}_{\text {th }}=\mathbb{C} 1$ (which is also invariant under all higher flows), thus this is assumed here for simplicity. Then, the projection onto $\mathcal{H}_{\text {th }}$ is just the subtraction of the average of the observable:

$$
\mathbb{P}_{\iota} a=\mathbf{1}\langle\mathbf{1}, a\rangle_{\mathcal{H}}=\mathbf{1}\langle a\rangle .
$$

That is, the thermodynamic space is nothing but the set of averages of local observables, and this indeed describes the thermodynamics. The presence of a higher-dimensional space of $\iota$-invariant is associated with phase co-existence, see for instance $[32,33,37]$. We now see that the condition (34), with respect to $U=\iota$, is simply that of strong enough clustering at large distances. Then $\langle a, b\rangle_{\mathcal{H}^{\prime}}$ is the space-integrated connected two-point function:

$$
\langle a, b\rangle_{\mathcal{H}^{\prime}}=\int \mathrm{d} x\left\langle a^{\dagger}(x) \cdot b(0)\right\rangle^{\mathrm{c}}=\left\langle A^{\dagger} \cdot b\right\rangle^{\mathrm{c}}:=\left\langle A^{\dagger} \cdot b\right\rangle-\left\langle A^{\dagger}\right\rangle\langle b\rangle
$$

where $a(x)=\iota_{x} a$ and I denote by capital letters the formal total space integrals, $\int \mathrm{d} x a(x)=$ $A$. In quantum spin chains, in any Gibbs state with respect to a local enough Hamiltonian, the clustering requirement (34) follows for local observables from a standard result of Araki [36], and as mentioned we may take $V^{\prime}=V$ for the nucleus.

Second, the space of invariants $\mathcal{H}_{\mathrm{bal}}$ is the space of conserved quantities of the model. Indeed, if observables $q$ and $j$ in the nucleus $V^{\prime}$ are related as per the continuity relation

$$
\partial_{t} q(x, t)+\partial_{x} j(x, t)=0
$$

where $a(x, t)=\iota_{x} \tau_{t} a$, then clearly $\partial_{t} q(x, t) \equiv \mathscr{H}^{\prime} 0$, as the action of $\iota_{x}$ on $\mathcal{H}^{\prime}$ is trivial. In fact, it is possible to show that if $q$ clusters fast enough and $\partial_{t} q(x, t) \equiv \mathscr{H}^{\prime} 0$, then there exists $j$, which is clustering, such that (48) holds; see [23] for the precise statement. Therefore, if $q$ satisfies (48), the equivalence class of $q$, an element of $\mathcal{H}^{\prime}$, lies in $\mathcal{H}_{\text {bal }}$,

$$
[q]_{\mathcal{H}^{\prime}} \in \mathscr{H}_{\text {bal }} \text {. }
$$

Under the equivalence, we have $q \equiv_{\mathcal{H}^{\prime}} q+\partial_{x} a$ for local $a$, and therefore it is natural to identify the equivalence class of $q$ with the formal, total space integral $Q=\int \mathrm{d} x q(x)$ (that is, $\int \mathrm{d} x \iota_{x}: \mathcal{V} \rightarrow \mathcal{H}^{\prime}$ ). This is a conserved quantity.

Denoting by $q_{i}$ a basis for $\mathcal{H}_{\text {bal }}$ (which is countable-dimensional), we have, on $\mathscr{H}^{\prime}$,

$$
\mathbb{P}_{\tau} a=\sum_{i} q_{i} C^{i j}\left\langle q_{j}, a\right\rangle_{\mathcal{H}^{\prime}}
$$

where $C^{i j}$ is the inverse of the static covariance matrix $C_{i j}=\left\langle q_{i}, q_{j}\right\rangle_{\mathcal{H}^{\prime}}$ (see Appendix 2). In particular, there are basis elements $q_{I}$ for $\mathscr{H}_{\text {bal }}$ that diagonalise the flux Jacobian, see Appendix 2; these are the normal modes, here labelled by capital letters $I, J, K, \ldots$.

Of interest is the large-time limit, or time-average, of overlaps where one observable is evolved in time. By von Neumann's ergodic theorem (see the precise statement in [23]), this projects onto $\mathcal{H}_{\mathrm{bal}}$,

$$
\mathrm{D}_{a, b}=\lim _{T \rightarrow \infty} \frac{1}{2 T} \int_{-T}^{T}\left\langle\tau_{t} a, b\right\rangle_{\mathscr{H}^{\prime}}=\langle a, b\rangle_{\mathrm{bal}} .
$$

If $a=j_{i}$ and $b=j_{j}$ are chosen to be currents associated to conserved densities as in (48), then $\mathrm{D}_{a, b}$ are the widely-studied Drude weights characterising ballistic transport [1]. Thus 
indeed $\mathcal{H}_{\mathrm{bal}}$ is associated to ballistic transport. In particular, we obtain the hydrodynamic projection formula for the Drude weights [1,21],

$$
\mathrm{D}_{a, b}=\sum_{i j}\left\langle a, q_{i}\right\rangle_{\mathrm{bal}} \mathrm{C}^{i j}\left\langle q_{j}, b\right\rangle_{\mathrm{bal}} .
$$

The inner product (47) and the associated Hilbert space $\mathcal{H}^{\prime}$ have been studied in the context of many-body systems [22]. The space of invariants $\mathscr{H}_{\text {bal }}$ can be shown rigorously to control the correlation functions at Euler scales [23].

Finally, what is the meaning of $\mathcal{H}^{\prime \prime}$, and in particular of the space of invariants $\mathscr{H}_{\text {dif }}$ of $\mathcal{H}^{\prime \prime}$ ? Of course, this invariant subspace is not the full space only if there are nontrivial higher flows, $N>2$. Recall that by construction $q_{i} \equiv_{\mathcal{H}^{\prime \prime}} 0$; hence these are not nontrivial elements of $\mathcal{H}_{\text {dif }}$. But we get intuition on $\mathcal{H}_{\text {dif }}$ by noting that, if $\left[j_{i}\right]_{\mathcal{H}^{\prime}}$, from the current defined in (48), projects fast enough onto $\mathcal{H}_{\mathrm{bal}}$ at large times, so that it lies in the nucleus $V^{\prime \prime}$, then the elements $\left[j_{i}\right]_{\mathscr{H}^{\prime \prime}} \in \mathscr{H}^{\prime \prime}$ lie in the subspace of invariants under any higher flows,

$$
\left[j_{i}\right]_{\mathcal{H}^{\prime \prime}} \in \mathscr{H}_{\text {dif }} .
$$

This is a result obtained in a different context [38], and reproduced in Appendix 3. The proof can be obtained in the $\mathscr{H}$-setup, and relies on the assumption that the conserved density $q_{i} \in V^{\prime}$, associated to $j_{i}$, satisfies continuity equations such as (48) but with other local currents $j_{i}^{(\ell)}$ when evolved under higher flows $\tau_{s}^{(\ell)}$. Then, we can re-write the inner product in $\mathscr{H}^{\prime \prime}$ as

$$
\mathfrak{L}_{a, b}=\langle a, b\rangle_{\mathcal{H}^{\prime \prime}}=\int \mathrm{d} t\left[\int \mathrm{d} x\left\langle a^{\dagger}(x, t) \cdot b(0,0)\right\rangle^{\mathrm{c}}-\mathrm{D}_{a, b}\right] .
$$

Taking again $a=j_{i}$ and $b=j_{j}$, Eq. (54) is nothing else but the Green-Kubo formula for the Onsager matrix, and we see that

$$
\mathfrak{L}_{i j}=\mathfrak{L}_{j_{i}, j_{j}}=\left\langle j_{i}, j_{j}\right\rangle_{\text {dif }} .
$$

As the Onsager matrix is simply related to the diffusion matrix [1] (see also [14]) ${ }^{3}$, this shows that $\mathscr{H}_{\text {dif }}$ is the Hilbert space encoding diffusive processes in hydrodynamics.

Much like in (52), we can expand in a basis $\left\{w_{\alpha}\right\}$ for $\mathcal{H}_{\mathrm{dif}}$,

$$
\langle a, b\rangle_{\text {dif }}=\sum_{\alpha, \beta}\left\langle a, w_{\alpha}\right\rangle_{\text {dif }} \mathfrak{C}^{\alpha \beta}\left\langle w_{\beta}, b\right\rangle_{\text {dif }},
$$

where $\mathfrak{C}^{\alpha \beta}$ is the inverse of the diffusive covariance matrix $\mathfrak{C}_{\alpha \beta}$,

$$
\mathfrak{C}_{\alpha \beta}=\left\langle w_{\alpha}, w_{\beta}\right\rangle_{\mathcal{H}^{\prime \prime}} .
$$

If we know the elements $w_{\alpha}$ and how to evaluate the components of $a$ and $b$ on these, we have an exact projection formula for the Onsager matrix. In order for this to be useful, we need to characterise the basis elements $w_{\alpha}$. Characterising the elements $w_{\alpha}$ is where all the physics of diffusion lies. In order to address this, I discuss the relation between the hydrodynamic spaces and extensive charges in Sect. 4.

Crucially, however, the clustering requirement (34) for $\mathcal{H}^{\prime}$, instead of $\mathcal{H}$, does not follow on local observables from standard results. The limit defining the Onsager matrix may not

\footnotetext{
3 In [14, Sec 2], the derivation is correct in the quantum case if products of local observables are replaced by the KMB product $\cdot$. This guarantees that the result of the formal $\beta_{j}(x)$-differentiation is real in [14, Eq B.3], and that $\mathscr{P} \mathcal{T}$-symmetry implies [14, Eq 2.33]. This then shows that (54) and (55) is indeed a good definition of the Onsager matrix elements in the quantum case.
} 
exist. Nevertheless, at least for the diagonal elements, one can always define the lower Onsager matrix elements using the lower norm (39), as

$$
\mathfrak{L}_{a, a}^{-}=\left(\|a\|_{\mathscr{H}^{\prime \prime}}^{-}\right)^{2}, \quad \mathfrak{L}_{i i}^{-}=\left(\left\|j_{i}\right\|_{\mathscr{H}^{\prime \prime}}^{-}\right)^{2} .
$$

These exist in the extended reals for all $a \in \mathcal{H}^{\prime}$ (including all $j_{i} \in \mathcal{H}^{\prime}$ ). It is the analysis of such lower norms, and their relations to extensive charges, that lead to nontrivial statements about diffusion and superdiffusion.

Remark 3.4 The phenomenology from nonlinear fluctuating hydrodynamics (NFH) [2] suggests that in typical non-integrable systems, clustering on $\mathcal{H}^{\prime}$ is not fast enough for many observables, including some (or all) conserved currents $j_{i}$, and that one has $\mathfrak{L}_{i i}^{-}=\infty$. In integrable systems, the Onsager matrix elements appear to be finite for a large class of currents [13-15], but not for all [9,16-20]. As part of the results of this work, sufficient conditions for lower Onsager matrix elements to be infinite are obtained, these conditions agreeing with the results of NFH.

Remark 3.5 The conserved densities $q_{i}$ can be considered as lying in various spaces: as local observables in $V$ and in fact of the nucleus $V^{\prime}$, or equivalence classes, elements of $\mathscr{H}^{\prime}$, spanning the subspace $\mathscr{H}_{\text {bal }}$. The latter is the more fundamental definition; however the former is more convenient for explicit calculations, and for considerations of the conservation laws (48). Naturally, an element $q_{i}$ of $\mathscr{H}^{\prime}$ can be defined from an element of $V^{\prime}$, but in general the converse may have obstructions ( $q_{i}$ may be a Cauchy sequence which converges in $\mathscr{H}^{\prime}$ but not in $\mathscr{H}$, or not in more general expectation values $\langle\cdots\rangle$ ) and ambiguities (shifts $q_{i}(x) \mapsto q_{i}(x)+a \mathbf{1}$ and $q_{i}(x) \mapsto q_{i}(x)+\partial_{x} a(x)$ modify the element of $\mathcal{V}$ or $V^{\prime}$, but not its equivalence class in $\left.\mathscr{H}^{\prime}\right)$.

Below, I will assume that $V \subset V^{\prime}$ (the local observables are clustering fast enough in space; see Remark 3.3), that the above ambiguities are the only ones (see Remark 3.1), and that it is possible to take a basis $q_{i} \in \mathscr{H}^{\prime}$ formed of equivalence classes of local observables (elements of $v$ ), and I will choose for every such $q_{i}$ a unique representative, still denoted $q_{i} \in \mathcal{V}$, which satisfies conservation laws (48) for local current observables with respect to time evolution $\tau_{t}$ and every higher flow $\tau_{s}^{(\ell)}$ (if any).

In non-integrable cases, $\mathcal{H}_{\text {bal }}$ is expected to be a finite-dimensional space generated by local observables. In integrable quantum chains, $V$ may have to include observables which are quasi-local, supported on infinitely-many sites but with appropriate decay properties [34].

Remark 3.6 It is usually the case that the conserved charges $Q_{i}=\int \mathrm{d} x q_{i}(x)$ generate flows on the observables in $\mathcal{H}$, with infinitesimal generator given, on a dense subspace, by the commutator (resp. Poisson bracket) in quantum (resp. classical) systems, i[ $\left.Q_{i}, \cdot \cdot\right]$. Further, it is also often the case that many, although not necessarily all, of these flows commute with each other.

\subsection{Maximal Entropy States and Hydrodynamic Equation}

The state $\langle\cdots\rangle$ has up to now been assumed to be invariant under the flows $\tau_{s}^{(\ell)}$, including space and time translations, and to have appropriate clustering properties, Eq. (34) and the various clustering assumptions in Sect. 5. A natural set of such states are those which have maximised entropy with respect to all available conserved charges. Their statistical distributions are formally of the form $\mathrm{e}^{-\sum_{i} \beta^{i} Q_{i}}$, where again $Q_{i}=\int \mathrm{d} x q_{i}(x)$ are the (formal) total conserved 
charges. A better definition is that the thermodynamic potentials $\beta^{i}$ generate a flow on the set of states, defined by the equation

$$
-\frac{\partial}{\partial \beta^{i}}\langle\cdots\rangle=\left\langle Q_{i} \cdots\right\rangle-\left\langle Q_{i}\right\rangle\langle\cdots\rangle .
$$

This can be taken as the starting point for a rigorous construction of the states, see [22]. These maximal entropy states include Gibbs and generalised Gibbs states. An immediate consequence is that variations in $\mathcal{H}_{\mathrm{th}}$ are related to overlaps in $\mathcal{H}_{\mathrm{bal}}$ :

$$
-\frac{\partial\langle a\rangle}{\partial \beta^{i}}=\left\langle q_{i}, a\right\rangle_{\mathcal{H}^{\prime}}=\left\langle q_{i}, a\right\rangle_{\mathcal{H}_{\text {bal }}} \text {. }
$$

Note in particular that the thermodynamic potentials $\beta^{i}$ are here defined in a gauge-invariant fashion (they do not depend on the specific choice of $q_{i}$ as an element of $\mathscr{H}$ ).

Within this formulation, one can write the hydrodynamic equations in an elegant form that makes clear the role of $\mathscr{H}_{\text {bal }}$ and $\mathscr{H}_{\text {dif }}$. Recall that the hydrodynamic equation is an equation for how the state $\langle\cdots\rangle_{x, t}$ depends on $x, t$, in the manifold of maximal entropy states. Using the state coordinates $\beta^{i}(x, t)$, up to diffusive scales, a form of the hydrodynamic equation is

$$
\sum_{j} \mathrm{C}_{i j} \partial_{t} \beta^{j}+\sum_{j}(\mathrm{AC})_{i j} \partial_{x} \beta^{j}=\frac{1}{2} \partial_{x}\left(\mathfrak{L}_{i j} \partial_{x} \beta^{j}\right)
$$

where $C_{i j}=\left\langle q_{i}, q_{j}\right\rangle_{\text {bal }}$ is the static correlation matrix, and $\mathrm{A}_{i}{ }^{j}=\partial\left\langle j_{i}\right\rangle / \partial\left\langle q_{j}\right\rangle$ is the flux Jacobian, with $(\mathrm{AC})_{i j}=\left\langle q_{i}, j_{j}\right\rangle_{\text {bal }}$. This can equivalently be written as

$$
\left\langle u+v, q_{i}\right\rangle_{\text {bal }}=\frac{1}{2} \partial_{x}\left\langle v, j_{i}\right\rangle_{\text {dif }}, \quad u=\sum_{i} q_{i} \partial_{t} \beta^{i}, v=\sum_{i} j_{i} \partial_{x} \beta^{i},
$$

where ballistic and diffusive terms are described by the ballistic and diffusive Hilbert spaces. In particular, the Euler equation is an equation on $\mathcal{H}_{\text {bal }}$,

$$
u+v=0 \quad \text { (on } \mathcal{H}_{\mathrm{bal}}, \text { at the Euler scale). }
$$

At the diffusive scale, the total hydrodynamic entropy production can be written as

$$
\partial_{t} S=\frac{1}{2} \int \mathrm{d} x\|\nu\|_{\mathrm{dif}}^{2},
$$

which is obviously non-negative. The diffusive Hilbert space also gives a measure of the distance of a space-time configuration to the Euler equation, for instance

$$
\int_{x_{1}}^{x_{2}} \mathrm{~d} x\left|\left\langle u+v, q_{i}\right\rangle_{\text {bal }}\right| \leq\left(N_{\left[x_{1}, x_{2}\right]}+1\right) \max _{\left[x_{1}, x_{2}\right]}\left(\|v\|_{\text {dif }}\left\|j_{i}\right\|_{\text {dif }}\right)
$$

where $N_{\left[x_{1}, x_{2}\right]}$ is the number of extrema of $\left\langle\mathfrak{v}, j_{i}\right\rangle_{\text {dif }}$ in the open interval $\left(x_{1}, x_{2}\right)$.

The hydrodynamic Eq. (62) is written in an explicitly gauge-invariant form: the inner products are gauge invariant, and the state coordinates $\beta^{i}$ also are. Thus, even up to diffusive scale, the hydrodynamic equation is universal: it does not depend on the choice of the conserved densities $q_{i}$ as elements of $\mathcal{H}$. In order to extract physical information from (62), however, one must provide a map from $\beta^{i}$ to averages of local observables in space-time. This map is not universal; in general, one chooses a set of densities $q_{i}$ in $\mathcal{H}$ and the map is completely fixed by requiring $\left\langle q_{i}(x, t)\right\rangle=\left\langle q_{i}\right\rangle_{x, t}$. 


\subsection{Fractional-Order Spaces and Superdiffusion}

It may happen that for some elements $a \in \mathcal{H}$ the requirement (34) is not satisfied: the vanishing is not fast enough for the integral (36) to exist. Consider the discussion of Sect. 3.2. It may be that $\|a\|_{\mathcal{H}^{\prime}}=\infty$. It is then natural to define Hilbert spaces at fractional orders, lying in a sense between $\mathscr{H}$ and $\mathscr{H}^{\prime}$. A proposal is, for $u \in(0,1)$,

$$
\langle a, b\rangle_{\mathscr{H}^{(u)}}=\lim _{n \rightarrow \infty} n^{u-1} \int_{-n}^{n} \mathrm{~d} x\left\langle\left(1-\mathbb{P}_{\iota}\right) \iota_{x} a, b\right\rangle_{\mathcal{H}} .
$$

More precisley one may consider elements for which the following asymptotic expansion holds

$$
\left\langle\left(1-\mathbb{P}_{\iota}\right) \iota_{x} a, b\right\rangle_{\mathcal{H}}=\omega_{a, b}^{ \pm}|x|^{-u}(1+o(1)) \quad(s \rightarrow \pm \infty)
$$

for some $\omega_{a, b}^{ \pm} \in \mathbb{C}$. Then,

$$
\langle a, b\rangle_{\mathcal{H}^{(u)}}=\frac{\omega_{a, b}^{+}+\omega_{a, b}^{-}}{1-u} .
$$

Non-negativity of the inner product is guaranteed by a similar argument as in (37):

$$
\begin{aligned}
& \lim _{n \rightarrow \infty}(2 n)^{u-2} \int_{-n}^{n} \mathrm{~d} x \int_{-n}^{n} \mathrm{~d} y\left\langle\left(1-\mathbb{P}_{\iota}\right) \iota_{x} a,\left(1-\mathbb{P}_{\iota}\right) \iota_{y} b\right\rangle_{\mathcal{H}} \\
& \quad=\lim _{n \rightarrow \infty}(2 n)^{u-2} \int_{-2 n}^{2 n} \mathrm{~d} x|2 n-x|\left\langle\left(1-\mathbb{P}_{\iota}\right) \iota_{x} a, b\right\rangle_{\mathcal{H}}=\frac{\omega_{a, b}^{+}+\omega_{a, b}^{-}}{(1-u)(2-u)}=\frac{\langle a, b\rangle_{\mathcal{H}^{(u)}}}{2-u} .
\end{aligned}
$$

The left-hand side is non-negative whenever $a=b$. A possible definition of the $u$ th-order space with respect to $\mathscr{H}$ is therefore as the completion $\mathscr{H}^{(u)}$ of the set of equivalence classes of a subspace $V^{(u)} \subset \mathcal{H}$ (the nucleus) in which (67) holds pairwise. Likewise, the $u$ th-order lower norm is, for any $a \in \mathcal{H}$,

$$
\|a\|_{\mathscr{H}^{(u)}}^{-}=\sqrt{2-u} \liminf _{n \rightarrow \infty}(2 n)^{\frac{u-2}{2}}\left\|\int_{-n}^{n} \mathrm{~d} x\left(1-\mathbb{P}_{\iota}\right) \iota_{x} a\right\|_{\mathcal{H}}(u \in(0,1)) .
$$

Note that there is at most one value of $u_{*} \in(0,1)$ such that $0<\|a\|_{\mathscr{H}^{\left(u_{*}\right)}}^{-}<\infty$, with ordering on the extended reals,

$$
\|a\|_{\mathscr{H}^{(u)}}^{-}=0\left(u<u_{*}\right), \quad\|a\|_{\mathscr{H}^{(u)}}^{-}=\infty\left(u>u_{*}\right) .
$$

The construction can be made based on $\mathscr{H}^{\prime}$ instead of $\mathcal{H}$. I will understand $\mathscr{H}^{(u)}$ for $u \in(j, j+1), j=1,2,3, \ldots$, as $(u-j)$ th-order spaces with respect to $\mathcal{H}^{(j)}$. For instance, for $u \in(1,2)$, there is a nucleus $\mathcal{V}^{(u)} \in \mathscr{H}^{\prime}$ where the asymptotic condition

$$
\left\langle\left(1-\mathbb{P}_{\tau}\right) \tau_{t} a, b\right\rangle_{\mathcal{H}^{\prime}}=\omega_{a, b}^{ \pm}|t|^{-(u-1)}(1+o(1)) \quad(t \rightarrow \pm \infty),
$$

holds; the inner product is

$$
\langle a, b\rangle_{\mathscr{H}^{(u)}}=\lim _{n \rightarrow \infty} n^{u-2} \int_{-n}^{n} \mathrm{~d} t\left\langle\left(1-\mathbb{P}_{\tau}\right) \tau_{t} a, b\right\rangle_{\mathscr{H}^{\prime}},
$$


and for every $a, b \in \mathcal{V}^{(u)}$; the space $\mathscr{H}^{(u)}$ is obtained by completing the space of equivalence classes, and the lower norm is

$$
\|a\|_{\mathcal{H}^{(u)}}^{-}=\sqrt{3-u} \liminf _{n \rightarrow \infty}(2 n)^{\frac{u-3}{2}}\left\|\int_{-n}^{n} \mathrm{~d} t\left(1-\mathbb{P}_{\tau}\right) \tau_{t} a\right\|_{\mathcal{H}^{\prime}}(u \in(1,2)) .
$$

For my purposes, the construction of $\mathscr{H}^{(u)}$ for $u \in(1,2)$ is the most relevant. By the calculation of Appendix 3, one shows that if a current observable $j_{i}$ has equivalence classes that lies in $\mathscr{H}^{(u)}$, then it lies in the subspace of invariants $\mathscr{Q}^{(u)} \subset \mathcal{H}^{(u)}$ under higher flows. This space of invariants is, in some sense, "in-between" the ballistic and diffusive spaces.

In Appendix 4, I argue that $\mathscr{H}^{(u)}$ (or more precisely, $2^{(u)}$ ) represents superdiffusion. More precisely, from considering the phenomenology of superdiffusive correlation functions in space-time, I obtain a precise definition of what characterises a superdiffusive mode. Consider a hydrodynamic normal mode $q_{I}$. If it is superdiffusive, one expects, at large spacetime separations,

$$
\left\langle q_{I}(x, t) q_{I}(0,0)\right\rangle^{\mathrm{c}} \sim \frac{1}{|\lambda t|^{\alpha}} f\left(\frac{x-v_{I}^{\mathrm{eff}} t}{|\lambda t|^{\alpha}}\right)
$$

where $f(z)>0$ integrates to $1, \lambda>0, v_{I}^{\text {eff }}$ is the eigenvalue associated to $q_{I}$, and $\alpha \in(1 / 2,1)$ (the case $\alpha=1 / 2$ would be normal diffusion). In fact, for a given $\alpha$, one should distinguish two types of superdiffusive behaviours, "normal" and "fat", according as the variance of the distribution $f(z)$ behaves: finite or infinite. In the latter case, an analysis of behaviours studied in the literature suggests a mechanism by which a precise power law emerges as a function of $\alpha$. Thus one has either

$$
\int \mathrm{d} z z^{2} f(z)<\infty \quad \text { (normal superdiffusion), }
$$

or, by the argument presented in Appendix 4,

$$
f(z) \sim c|z|^{-\left(1+\frac{1}{\alpha}\right)} \quad \text { (fat superdiffusion) }
$$

for some $c>0$, at large $|z|$. In Appendix 4 I then explain how, using conservation laws, Eq. (75), and these considerations about the variance of $f(z)$, the following conditions on the lower norm (74) of the associated current $j_{I}$ are obtained:

$$
\left\|j_{I}\right\|_{\mathcal{H}^{(u)}}^{-}=\left\{\begin{array}{ll}
0 & \text { for } u<3-2 \alpha \\
\infty & \text { for } u>3-2 \alpha
\end{array}\right. \text { (normal superdiffusion) }
$$

and

$$
\left\|j_{I}\right\|_{\mathcal{H}^{(u)}}^{-}=\left\{\begin{array}{ll}
0 & \text { for } u<1 / \alpha \\
\infty & \text { for } u>1 / \alpha
\end{array}\right. \text { (fat superdiffusion). }
$$

These are taken here as precise definitions of normal and fat superdiffusive modes. That is, a current observable $j_{I} \in \mathcal{H}^{\prime}$ has normal (fat) superdiffusion exponent $\alpha$ if Eq. (78) [(Eq. (79)] holds. These conditions will form the basis for the results on superdiffusion in the following sections. 


\section{Extensive Charges and Lower Bounds on Diffusion and Superdiffusion}

Extensive charges are sequences of observables which represent, in a more concrete fashion, the equivalence classes discussed in the previous section. They serve here two purposes: first to make the abstract hydrodynamic Hilbert spaces more concrete, and second to provide a way of bounding (from below) physical quantities of interest, in particular the Onsager matrix elements and superdiffusion exponents.

\subsection{Linearly Extensive Charges}

Let me consider the general setup of Sect. 3.1, the reduction $\mathscr{H} \stackrel{U}{\rightarrow} \mathscr{H}^{\prime}$ with nucleus $V^{\prime}$. The concept of extensive charges emerged from Prosen's work on pseudolocal charges [39,40], and was developed in integrable spin chains [27,28]. I gave a precise construction in [22] in the context of quantum spin chains. I follow these lines here, and define linearly extensive charges in the present more general context.

Consider a sequence $Q=\left(Q_{n} \in \mathcal{H}: n \in \mathbb{N}\right)$. Assume without loss of generality that $Q_{n}=(1-\mathbb{P}) Q_{n}$ for all $n$. Suppose there is a subspace $\mathscr{D}^{\prime}(Q) \subset \mathcal{H}$, the domain of $Q$, with $V^{\prime} \subset \mathscr{D}^{\prime}(Q)$, such that the sequence satisfies the following conditions:

1. There exists $\gamma>0$ such that $\left\|Q_{n}\right\|_{\mathscr{H}}^{2} \leq \gamma n$ for all $n \in \mathbb{N}$ large enough.

2. The limit $Q^{\prime}(a):=\lim _{n \rightarrow \infty}\left\langle Q_{n}, a\right\rangle_{\mathcal{H}}$ exists for all $a \in \mathscr{D}^{\prime}(Q)$.

3. There exists $k>0$ such that $\lim _{n \rightarrow \infty} \sup _{s, t \in[-k n, k n]}\left|\left\langle Q_{n}, U_{s} a-U_{t} a\right\rangle_{\mathcal{H}}\right|=0$ for all $a \in \mathscr{D}^{\prime}(Q)$.

A linearly extensive charge for $\mathcal{H}$ is an equivalence class of such sequences, under the equivalence $Q^{\prime} \equiv \tilde{Q}^{\prime}$ if and only if $Q^{\prime}(a)=\tilde{Q}^{\prime}(a) \forall a \in V^{\prime}$. That is, the set of such limit-actions of such sequences is the set of extensive charges.

The first point above states the linear extensivity of the charge. The third specifies that the charge is extensive with respect to $U_{s}$, and that it is homogeneous. The second specifies that it acts well on $\mathscr{D}^{\prime}(Q)$. I emphasise that there is here no condition on these charges being "conserved": in the general setup there is no such notion, as only the one-parameter group $U$ is considered.

One can show (see below) that $Q^{\prime}(\cdot)$ is bounded with respect to the lower norm on $\mathcal{H}^{\prime}$. As a consequence, on $\mathcal{V}^{\prime}$ it is continuous with respect to the norm on $\mathcal{H}^{\prime}$, and by the Riesz representation theorem, there exists $b \in \mathscr{H}^{\prime}$ such that

$$
Q^{\prime}(a)=\langle b, a\rangle_{\mathcal{H}^{\prime}} \quad \forall a \in V^{\prime} .
$$

Since $V^{\prime}$ is dense in $\mathscr{H}^{\prime}$, the map can be extended, by continuity, to all of $\mathscr{H}^{\prime}$.

The proof of boundedness is simple. I reproduce that of [22]. Let $a \in \mathscr{D}^{\prime}(Q)$. By Point 3 above and the assumption that $Q_{n}=(1-\mathbb{P}) Q_{n}$, there exists $\delta_{n}$ with $\lim _{n \rightarrow \infty} \delta_{n}=0$, such that

$$
\left\langle Q_{n}, a\right\rangle_{\mathcal{H}}=\frac{1}{2 k n} \int_{-k n}^{k n} \mathrm{~d} s\left\langle Q_{n},(1-\mathbb{P}) U_{s} a\right\rangle_{\mathscr{H}}+\delta_{n} .
$$

The norm of the first term on the right-hand side is bounded by using the Cauchy-Schwartz inequality:

$$
\leq \sqrt{\frac{\left\|Q_{n}\right\|_{\mathcal{H}}^{2}}{2 k n}} \sqrt{\frac{1}{2 k n} \int_{-k n}^{k n} \mathrm{~d} s \int_{-k n}^{k n} \mathrm{~d} t\left\langle(1-\mathbb{P}) U_{s} a,(1-\mathbb{P}) U_{t} a\right\rangle_{\mathcal{H}}}
$$


and by using (39), we obtain

$$
\left|Q^{\prime}(a)\right| \leq \sqrt{\frac{\gamma}{2 k}}\|a\|_{\mathcal{H}^{\prime}}^{-}
$$

If $a \in \mathcal{V}^{\prime}$ then we can use $\|a\|_{\mathcal{H}^{\prime}}^{-}=\|a\|_{\mathscr{H}^{\prime}}$ the norm on $\mathscr{H}^{\prime}$. With (80) we find

$$
\|\mathcal{G}\|_{\mathcal{H}^{\prime}} \leq \sqrt{\frac{\gamma}{2 k}} .
$$

Simple examples of such extensive charges are the (equivalence classes of the) sequences of the form

$$
Q_{n}=\int_{-n / 2}^{n / 2} \mathrm{~d} s U_{s} b
$$

for $b \in V^{\prime}$, where one can take any $0<k<1 / 2$. In this simple case, the limit action is $Q^{\prime}(a)=\langle\mathfrak{b}, a\rangle_{\mathcal{H}^{\prime}}$, and the norm is exactly $\|\mathfrak{b}\|_{\mathcal{H}^{\prime}}=\sqrt{\gamma}$.

In [22], this construction was used with $U=\iota$ space translations. It was also shown that there is in fact a bijection between the set of linearly extensive charges, and the Hilbert space $\mathcal{H}^{\prime}$. The proof can be applied to the general construction presented here as well. Therefore

$$
\text { linearly extensive charges for } \mathcal{H} \cong \mathcal{H}^{\prime} \text {. }
$$

This extends the second equation of (47) to arbitrary linearly extensive charges $Q^{\prime}$ instead of the particular $A=\int \mathrm{d} x a(x)$ constructed from elements $a \in V^{\prime}$. This establishes that the correct completion of the space of such formal integrals of local densities is the Hilbert space $\mathcal{H}^{\prime}$. I will not need the isomorphism (86) in what follows.

\subsection{Quadratically Extensive Charges}

Now concentrate on the specific setup of Sects. 3.2 and 3.3. There are (at least) two oneparameter groups, space translations $\iota_{x}$ and time translations $\tau_{t}$. As the construction in Sect. 4.1 is completely general, we can apply it for $U=\iota$ with $\mathbb{P}=\mathbb{P}_{\iota}$, and this is how we will understand Points 1, 2, 3. But also we can go further and construct the linearly extensive charges for $\mathscr{H}^{\prime}$. We consider a sequence $Q=\left(Q_{n} \in \mathcal{H}^{\prime}: n \in \mathbb{N}\right)$, and assume without loss of generality that $Q_{n}=\left(1-\mathbb{P}_{\tau}\right) Q_{n}$ for all $n$. We suppose there is a subspace $\mathscr{D}^{\prime \prime}(Q) \subset \mathcal{H}^{\prime}$, the domain of $Q$, with $V^{\prime \prime} \subset \mathscr{D}^{\prime \prime}(Q)$, such that the sequence satisfies the following conditions:

$1^{\prime}$. There exists $\gamma>0$ such that $\left\|Q_{n}\right\|_{\mathcal{H}^{\prime}}^{2} \leq \gamma n$ for all $n \in \mathbb{N}$ large enough.

$2^{\prime}$. The limit $Q^{\prime \prime}(a):=\lim _{n \rightarrow \infty}\left\langle Q_{n}, a\right\rangle_{\mathcal{H}^{\prime}}$ exists for all $a \in \mathscr{D}^{\prime \prime}(Q)$.

$3^{\prime}$. There exists $k>0$ such that $\lim _{n \rightarrow \infty} \sup _{s, t \in[-k n, k n]}\left|\left\langle Q_{n}, \tau_{s} a-\tau_{t} a\right\rangle_{\mathcal{H}^{\prime}}\right|=0$ for all $a \in \mathscr{D}^{\prime \prime}(Q)$.

By the general result of Sect. 4.1, every such charge $Q^{\prime \prime}$ is bounded on its domain by the lower norm for $\mathcal{H}^{\prime}$, and gives rise, when acting on $V^{\prime \prime}$, to an element of $\mathcal{H}^{\prime \prime}$. In fact, the space of these is isomorphic to $\mathcal{H}^{\prime \prime}$ (which I again state without proof as this will not be used). I will refer to these as the quadratically extensive conserved charges for $\mathcal{H}$ :

$$
\begin{aligned}
& \text { quadratically extensive conserved charges for } \mathcal{H} \\
= & \text { linearly extensive charges for } \mathcal{H}^{\prime} \\
\cong & \mathcal{H}^{\prime \prime} .
\end{aligned}
$$


For every such charge, there exists $b \in \mathcal{H}^{\prime \prime}$ such that

$$
Q^{\prime \prime}(a)=\langle b, a\rangle_{\mathcal{H}^{\prime \prime}}
$$

for all $a \in V^{\prime \prime}$. Note that these are "conserved" by construction, as in $\mathcal{H}^{\prime \prime}$, the group $\tau_{t}$ acts trivially. Naturally, sequences of the form $Q_{n}=\int_{-n / 2}^{n / 2} \mathrm{~d} t \tau_{t} b$ for $b \in \mathcal{V}^{\prime \prime}$ give rise to linearly extensive charges for $\mathcal{H}^{\prime}$. Most interestingly, we obtain a bound on the $\mathcal{H}^{\prime \prime}$-lower norm-or Onsager matrix element—of elements of $\mathscr{D}^{\prime \prime}(Q)$ on which $Q^{\prime \prime}$ is nonzero:

Theorem 4.1 Let $Q^{\prime \prime}$ be a quadratically extensive charge for $\mathcal{H}$, and $a \in \mathscr{D}^{\prime \prime}(Q)$. Then

$$
\mathfrak{L}_{a, a}^{-} \geq \frac{2 k}{\gamma}\left|Q^{\prime \prime}(a)\right|^{2} .
$$

If $j_{i} \in \mathscr{D}^{\prime \prime}(Q)$ and $Q^{\prime \prime}\left(j_{i}\right) \neq 0$, then the associated lower Onsager matrix element $\mathcal{L}_{i i}^{-}$is strictly positive (and may be infinite).

Proof This follows immediately from the above discussion and (83).

Why should linearly extensive charges for $\mathscr{H}^{\prime}$ be seen as quadratically extensive in $\mathscr{H}$ ? Intuitively, extensivity for $\mathcal{H}^{\prime}$ requires, from the viewpoint of $\mathcal{H}$, an integral on an interval of length $n$, whose position is integrated over the full space. As $n$ goes to infinity, we have a double integral over space. This will be made clearer in Sect. 5, where explicit examples are discussed. In particular, there, it is seen that, with $Q_{n}$ supported on regions of length $n$, and if a Lieb-Robinson bound is available,

$$
0<k<\frac{1}{2 v_{\mathrm{LR}}} \quad\left(Q_{n} \text { on length } n\right. \text { in space, Lieb-Robinson bound). }
$$

Another reason for the nomenclature lies in a construction by Prosen [10], which I show in Appendix 7 leads to elements of $\mathcal{H}^{\prime \prime}$. This construction appears to be slightly more restrictive than the general definition of quadratically extensive conserved charges that I propose in (87), because it requires an apparently stronger clustering property, Eq. (212). It is nevertheless useful in practice.

\subsection{Fractionally Extensive Charges}

There is a natural way of modifying the construction of linearly extensive charges of Sect. 4.1 in order to connect them to the fractional-order spaces of Sect. 3.5. I define $u$-extensive charges for $\mathcal{H}$ by assuming Point $1^{(u)}=1$, and modifying Points 2 (for the notation) and 3 (more fundamentally) as follows, for $u \in(0,1)$ :

$2^{(u)}$. The limit $Q^{(u)}(a):=\lim _{n \rightarrow \infty}\left\langle Q_{n}, a\right\rangle_{\mathcal{H}}$ exists for all $a \in \mathscr{D}^{(u)}(Q)$.

$3^{(u)}$. There exists $k>0$ such that $\lim _{n \rightarrow \infty} \sup _{x, y \in\left[-k n^{1 / u}, k n^{1 / u}\right]}\left|\left\langle Q_{n}, \iota_{x} a-\iota_{y} a\right\rangle_{\mathscr{H}}\right|=0$ for all $a \in D^{(u)}(Q)$.

We can bound the resulting charge by using

$$
\left\langle Q_{n}, a\right\rangle_{\mathscr{H}}=\frac{1}{2 k n^{1 / u}} \int_{-k n^{1 / u}}^{k n^{1 / u}} \mathrm{~d} x\left\langle Q_{n},\left(1-\mathbb{P}_{\iota}\right) \iota_{x} a\right\rangle_{\mathcal{H}}+\delta_{n}
$$

and by bounding the first term as

$$
\leq \sqrt{\frac{\left\|Q_{n}\right\|_{\mathcal{H}}^{2}}{4 k^{2} n}} \sqrt{\frac{1}{n^{2 / u-1}} \int_{-k n^{1 / u}}^{k n^{1 / u}} \mathrm{~d} x \int_{-k n^{1 / u}}^{k n^{1 / u}} \mathrm{~d} y\left\langle\left(1-\mathbb{P}_{\iota}\right) \tau_{x} a,\left(1-\mathbb{P}_{\iota}\right) \tau_{y} a\right)_{\mathcal{H}} .}
$$


Replacing $n$ by $k n^{1 / u}$ in (70), we obtain

$$
\left|Q^{(u)}(a)\right| \leq \frac{(2 k)^{-u / 2} \sqrt{\gamma}}{\sqrt{2-u}}\|a\|_{\mathcal{H}^{(u)}}^{-} .
$$

Thus, there exists $b \in \mathscr{H}^{(u)}$ such that, for all $a \in V^{(u)}$,

$$
Q_{\mathscr{H}^{(u)}}(a)=\langle\mathcal{b}, a\rangle_{\mathscr{H}^{(u)}}, \quad\|\mathcal{b}\|_{\mathscr{H}^{(u)}} \leq \frac{(2 k)^{-u / 2} \sqrt{\gamma}}{\sqrt{2-u}} .
$$

Again one can make a similar construction one level up: I define, for $u \in(1,2)$, the $u$-extensive charges for $\mathscr{H}$ as the $(u-1)$-extensive charges for $\mathscr{H}^{\prime}$. That is, Point $1^{(u)}=1^{\prime}$ holds, and, for $u \in(1,2)$,

$2^{(u)}$. The limit $Q^{(u)}(a):=\lim _{n \rightarrow \infty}\left\langle Q_{n}, a\right\rangle_{\mathcal{H}^{\prime}}$ exists for all $a \in \mathscr{D}^{(u)}(Q)$.

$3^{(u)}$. There exists $k>0$ such that $\lim _{n \rightarrow \infty} \sup _{s, t \in\left[-k n^{1 /(u-1)}, k n^{1 /(u-1)}\right]}\left|\left\langle Q_{n}, \tau_{s} a-\tau_{t} a\right\rangle_{\mathcal{H}^{\prime}}\right|=$ 0 for all $a \in \mathscr{D}^{(u)}(Q)$.

We obtain

$$
\left|Q^{(u)}(a)\right| \leq \frac{(2 k)^{-(u-1) / 2} \sqrt{\gamma}}{\sqrt{3-u}}\|a\|_{\mathscr{H}^{(u)}}^{-}
$$

and the following:

Theorem 4.2 Let $Q^{(u)}$ be a $u$-extensive charge for $\mathcal{H}$ with $u \in(1,2)$, and a $\in \mathscr{D}^{(u)}(Q)$. Then

$$
\|a\|_{\mathscr{H}^{(u)}}^{-} \geq \frac{(2 k)^{(u-1) / 2} \sqrt{3-u}}{\sqrt{\gamma}}\left|Q^{(u)}(a)\right| .
$$

If $j_{I} \in \mathscr{D}^{(u)}(Q)$ and $Q^{(u)}\left(j_{I}\right) \neq 0$, and if $j_{I}$ has normal (resp. fat) superdiffusion exponent $\alpha, E q$. (78) [resp. Eq. (79)], then this exponent is bounded as

$$
\alpha \geq \frac{3-u}{2} \quad\left(\operatorname{resp} . \alpha \geq \frac{1}{u}\right) .
$$

\section{Bilinear Charges}

The construction of Sect. 4, and in particular the bounds obtained, are still relatively abstract. Concrete bounds are obtained by choosing appropriate extensive charges. In this section, I construct such charges and analyse their consequences. The strength of the bound obtained depends on the assumption we can make on clustering properties of multi-point correlation functions. I will make in turn various assumptions increasing in strength, obtaining stronger results: basic clustering properties which follow from Lieb-Robinson bounds, stronger properties based on hydrodynamic velocities obtained by a linear response argument, and a still stronger clustering assumption from the superdiffusive phenomenology. The latter will give lower bounds on the superdiffusive exponents, reproducing that of the KPZ universality class and other exponents found in NFH. Under the clustering assumptions stated, and within the appropriate $C^{*}$-algebraic framework, the results are rigorous; however the clustering assumptions based on hydrodynamic velocities seem to be rather hard to show rigorously.

For simplicity, here and in the rest of the paper, I assume that $q_{i}^{\dagger}=q_{i}$, and that the state $\langle\cdots\rangle$ is invariant under the flows generated by all conserved charges $Q_{i}$; these assumptions are however not necessary for similar results to hold. 


\subsection{Bilinear Charges}

We are looking for a natural space of quadratically or fractionally extensive conserved charges for $\mathcal{H}$, as per the general definitions Points $1^{\prime}, 2^{\prime}, 3^{\prime}$, or Points $1^{(u)}, 2^{(u)}, 3^{(u)}$ with $u \in(1,2)$. Quadratically extensive charges for $\mathcal{H}$ are linearly extensive for $\mathcal{H}^{\prime}$, and with linear extensivity in time, the sequences of time integrals $\int_{-n}^{n} \mathrm{~d} t \tau_{t} a$ for $a \in \mathcal{V}$ [as in (85)], are examples. However these do not immediately lead to new results. Instead, we use the general construction and consider charges which are linearly extensive for $\mathcal{H}^{\prime}$ because of a linear growth in space. Certainly, because of Point $3^{\prime}$, they still must be invariant under time translation, hence these must be conserved charges.

A natural guess are the bilinear expressions in the total conserved charges, formally $Q_{i} Q_{j}$. This closely resembles the quadratic expansion of the current made in [29] following the philosophy of NFH, and I will establish the full connection in Sect. 6. Defining precise objects in $\mathscr{H}^{\prime}$, let me then consider the sequence in $n$, parametrised by $i, j$, defined as

$$
Q_{i j ; n}=\left(1-\mathbb{P}_{\tau}\right)\left[\int_{-n}^{n} \mathrm{~d} x q_{i}(x) q_{j}\right]_{\mathcal{H}^{\prime}}
$$

where $q_{i}(x)=\iota_{x} q_{i}$ is the space-translate, and $\mathbb{P}_{\tau}$ is the projector onto the space of conserved densities, Eq. (50). I show below that these indeed result in quadratically and fractionally extensive charges. They will be referred to as "bilinear charges", as they are formed of bilinear expressions in linearly extensive charges for $\mathscr{H}$.

In this section I assume, without loss of generality, that $q_{i}$ 's have zero expectation values $\left\langle q_{i}\right\rangle=0$.

Remark 5.1 In definition (98), the $q_{i}$ 's are seen as elements of $v$, the set of local observables, on which there is an algebra. The result of the product $q_{i}(x) q_{j}=q_{i}(x) q_{j}(0)$ is then seen as an element of $\mathscr{H}^{\prime}$ (that is, its equivalence class is taken), and then the projection $1-\mathbb{P}_{\tau}$ is applied on its integral. It will be simple to see that the resulting charge $Q_{i j}$ from (98) does not depend on the choice of element $q_{i}$ of the $\mathscr{H}^{\prime}$-equivalence class. Therefore, $Q_{i j}$ is indeed a bilinear in the charges $Q_{i}, Q_{j}$. The constant-shift ambiguity is immediately irrelevant: since $\mathbb{P}_{\tau} q_{i}=q_{i}$, we can replace $q_{i}$ by $q_{i}-\mathbf{1}\left\langle q_{i}\right\rangle$ in (98). For the gauge transformation, this follows from the clustering assumption (99) below. See Remark 3.5 for the assumptions made on the $q_{i}$ 's.

Remark 5.2 Below, the projection formula (50) is used, and assumed to converge, on local observables $a \in V$ inside correlation functions. Further, the clustering condition (99) is assumed to hold uniformly enough on a basis of conserved densities $q_{i}$. For simplicity, the flux Jacobian is also assumed to have discrete spectrum, and sums over normal modes are assumed to converge. These assumptions would be difficult to show rigorously, of course, only if $\mathcal{H}_{\text {bal }}$ is infinite-dimensional.

\subsection{Diffusion from Lieb-Robinson-Type Correlations}

In this subsection, I obtain bounds on diagonal Onsager matrix elements using the charges (98), by assuming a clustering condition on correlation functions. The idea behind the clustering assumption is the Lieb-Robinson bound, but the assumption is weak enough in order for the theorem to be as widely applicable as possible.

I assume the existence of $v>0$ such that, for all $a_{i} \in \mathcal{V}$, connected correlation functions $\left\langle a_{1}\left(x_{1}, t_{1}\right) a_{2}\left(x_{2}, t_{2}\right) \cdots\right\rangle^{\mathrm{c}}$ decay fast enough whenever there is some $i$ such that $\left|x_{i}-x_{j}\right|-$ 
$v\left|t_{i}-t_{j}\right| \gg 0$ for all $j$. For the proofs below, it is sufficient to restrict ourselves to connected correlation functions with up to four observables. More precisely, given $a_{i} \in \mathcal{V}$ for $i=$ $1, \ldots, r$, there exists a bounded function $f(z)>0(z \in \mathbb{R})$, and real numbers $b>0, c>0$ and $d>r-1$, such that

$$
\begin{array}{r}
\left|\left\langle a_{1}\left(x_{1}, t_{1}\right) \cdots a_{r}\left(x_{r}, t_{r}\right)\right\rangle^{\mathrm{c}}\right| \leq f\left(\max _{i}\left\{\min _{j}\left\{z_{i j}\right\}\right\}\right) \\
z_{i j}=\left|x_{i}-x_{j}\right|-v\left|t_{i}-t_{j}\right|, \quad f(z)=c(b+z)^{-d} \forall z>0 .
\end{array}
$$

Here and below, I use again the notation $a(x)=\iota_{x} a$ and $a(x, t)=\iota_{x} \tau_{t} a$.

This assumption naturally encodes the uniform boundedness of connected correlation functions via the introduction of the shift $b>0$, and their decay whenever any one of the observable is far enough in space. The decay occurs as soon as the maximal "extent" of the time-evolved observable, as determined by the velocity $v$, does not overlap with that of any of the other observables. If a Lieb-Robinson bound exists, with $v_{\mathrm{LR}}$ the Lieb-Robinson velocity, then it is expected that we can take any $v=v_{\mathrm{LR}}$ and $d$ arbitrary large; in fact, $f(z)$ may be chosen to decay exponentially at large $z$. This is rigorously proven in the case $r=2$ using the Lieb-Robinson bound for states which cluster in space exponentially [31,41], and general results exist for algebraically clustering states [22]; the proof techniques are generalisable to $r>2$. Note that (34), for $U_{s}$ being space translations, is exactly the special, non-dynamical case $r=2$ and $t_{1}=t_{2}$ of (99), hence we may take

$$
v^{\prime}=v .
$$

To be precise, below, we only need the non-dynamical case of (99) (with equal times) for $r=2,4$, and the dynamical case with $t_{1}=t_{2} \neq t_{3}$ for $r=3$. In the latter case, the bound is represented by Fig. 1, with the linear (ballistic) scaling $g(t) \propto t$, characteristic of the Lieb-Robinson bound.

It is simple to show that (99) implies that the equal-time, space-integrated $r$-point correlation functions are finite. For instance, the following integral on $\mathbb{R}^{2}$ exists and is finite, the condition $d>2$ being sufficient:

$$
\begin{aligned}
\left\langle A_{1} A_{2} a_{3}\right\rangle^{\mathrm{c}} & =\int \mathrm{d} x_{1} \mathrm{~d} x_{2}\left\langle a_{1}\left(x_{1}\right) a_{2}\left(x_{2}\right) a_{3}\left(x_{3}\right)\right\rangle^{\mathrm{c}} \\
& =\int_{z_{1}>z_{2}>0} \mathrm{~d} z_{1} \mathrm{~d} z_{2} \sum_{\sigma \in S_{3}}\left\langle: a_{\sigma_{1}}\left(z_{1}\right) a_{\sigma_{2}}\left(z_{2}\right) a_{\sigma_{3}}(0):\right\rangle^{\mathrm{c}}
\end{aligned}
$$

where the "normal ordering" $: \cdots$ : places the observables in the order $a_{1}(\cdot) a_{2}(\cdot) a_{3}(\cdot)$. Using (99),

$$
\begin{aligned}
\left\langle A_{1} A_{2} a_{3}\right\rangle^{\mathrm{c}} & \leq 6 c \int_{z_{1}>z_{2}>0} \mathrm{~d} z_{1} \mathrm{~d} z_{2}\left[b+\max \left\{z_{2},\left|z_{1}-z_{2}\right|\right\}\right]^{-d} \\
& =12 c \int_{z>w>0} \mathrm{~d} z \mathrm{~d} w(b+z)^{-d} \\
& \leq 12 c \int_{z>0} \mathrm{~d} z(b+z)^{1-d} \\
& =\frac{12 c b^{2-d}}{d-2}<\infty \text { for } d>2
\end{aligned}
$$

Using Theorem 4.1, I will show the following: 
Theorem 5.1 Let $a \in \mathcal{V}$. Under the assumption (99), we have

$$
\mathfrak{L}_{a, a}^{-} \geq \frac{\left|\left\langle Q_{i} Q_{j}\left(1-\mathbb{P}_{\tau}\right) a\right\rangle^{\mathrm{c}}\right|^{2}}{2 v\left(\left\|q_{i}\right\|_{\mathscr{H}^{\prime}}^{2}|| q_{j} \|_{\mathcal{H}^{\prime}}^{2}+\left\langle q_{i}, q_{j}\right\rangle_{\mathcal{H}^{\prime}}^{2}\right)}
$$

for any $i, j$. In particular, if

$$
\left\langle Q_{i} Q_{j} j_{k}\right\rangle^{\mathrm{c}}-\sum_{l m}\left\langle Q_{i} Q_{j} q_{l}\right\rangle^{\mathrm{c}} C^{l m}\left\langle Q_{m} j_{k}\right\rangle^{\mathrm{c}} \neq 0
$$

for some $i, j$, then the lower Onsager matrix element $\mathfrak{L}_{k k}^{-}$is strictly positive (and may be infinite).

All quantities in Theorem 5.1 can be evaluated purely from the thermodynamics of the system. This implies nonzero diffusion whenever (104) holds.

Consider for instance a conventional Galilean gas in a Gibbs state at rest. There are three conserved charges: the total number of particles $Q_{0}$, the total momentum $Q_{1}$ and the total energy $Q_{2}$, and the weight of a configuration takes the form $\exp \left[-\beta\left(Q_{2}-\mu Q_{0}\right)\right]$. Diffusion, characterised by the Onsager matrix element $\mathfrak{L}_{11}$, may be bounded by taking $k=1$ ( $j_{k}$ being the momentum current, that is, the pressure) and $i=0, j=0$ (the quadratic charge corresponding to the square of the total number of particles). Generically, the inequality (104) holds as basic symmetries do not forbid it, hence there is a nontrivial lower bound. The bound (103) can in fact be written in terms of conventional thermodynamic quantities. For simplicity, let us assume that all average quantities (densities of particles and energy, and pressure) vary very little with the inverse temperature $\beta$. Then we can concentrate solely on $Q_{0}$ and $Q_{1}$, keeping $\beta$ fixed, and $\mathrm{C}$ is a 2 by 2 matrix. By parity symmetry, the only element that contributes to the sum in (104) is $C^{00}$. Let $P=\left\langle j_{1}\right\rangle$ be the pressure, $\rho=\left\langle q_{0}\right\rangle$ the particle density, and $\chi=\beta^{-1} \partial \rho / \partial \mu$ the susceptibility. Note that $\mathrm{d} P / \mathrm{d} \rho=v_{\mathrm{s}}^{2}$ is the square of the sound speed. Then

$$
\mathfrak{L}_{1,1}^{-} \geq \frac{\left(\chi v_{\mathrm{s}} \partial_{\rho} v_{\mathrm{s}}\right)^{2}}{v} .
$$

Recall that $v$ is the velocity controlling the decay of correlation functions, which can be taken as the Lieb-Robinson velocity. The Onsager matrix element $\mathfrak{L}_{11}$ is simply related to the bulk viscosity $\zeta$ of the gas, as

$$
\zeta=\frac{\rho \mathfrak{L}_{1,1}}{2 \chi v_{\mathrm{s}}^{2}}
$$

Hence this gives the explicit positive lower bound on the viscosity (1), solely derived from clustering properties.

By nonlinear fluctuating hydrodynamics, diffusion is in fact expected to be infinite in many non-integrable systems, as there is superdiffusion; hence the above inequality is not expected to be saturated. Bounds on superdiffusion are discussed in Sects. 5.3 and 5.4.

Proof I show that the sequence $Q_{i j}=\left(Q_{i j ; n}: n \in \mathbb{N}\right)$ satisfies Points $1^{\prime}, 2^{\prime}$ and $3^{\prime}$ (Sect. 4.2) of linearly extensive charges for $\mathscr{H}^{\prime}$, giving the charge $Q_{i j}^{\prime \prime}$ with $\mathcal{C} \subset \mathscr{D}^{\prime \prime}\left(Q_{i j}\right)$.

Point $1^{\prime}$ is the requirement that $\left\|Q_{i j ; n}\right\|_{\mathscr{H}^{\prime}}^{2} \leq \gamma n$. We have, explicitly subtracting the projection onto conserved densities, 
$\left\|Q_{i j ; n}\right\|_{\mathscr{H}^{\prime}}^{2}=\int_{-n}^{n} \mathrm{~d} x \int_{-n}^{n} \mathrm{~d} y\left[\left\langle q_{i}(x) q_{j}, q_{i}(y) q_{j}\right\rangle_{\mathcal{H}^{\prime}}-\sum_{K}\left\langle q_{i}(x) q_{j}, q_{K}\right\rangle_{\mathcal{H}^{\prime}}\left\langle q_{K}, q_{i}(y) q_{j}\right\rangle_{\mathcal{H}^{\prime}}\right]$

where I used the diagonal basis $q_{K}$ described in Appendix 2. The second term in the square brackets on the right-hand side is finite under integration as $n \rightarrow \infty$ by three-point function clustering (99), and $\int \mathrm{d} x\left\langle q_{i}(x) q_{j}, q_{K}\right\rangle_{\mathcal{H}^{\prime}}=\left\langle Q_{i} Q_{j} \cdot q_{K}\right\rangle^{\mathrm{c}}$. Recall that we assume $\left\langle q_{i}\right\rangle=0$, thus the connected three-point functions appear. The first term in (107) is

$$
\int_{-n}^{n} \mathrm{~d} x \int_{-n}^{n} \mathrm{~d} y \int \mathrm{d} z\left[\left\langle q_{i}(z+x) q_{j}(z) \cdot q_{i}(0) q_{j}(y)\right\rangle-\left\langle q_{i}(z+x) q_{j}(z)\right\rangle\left\langle q_{i}(0) q_{j}(y)\right\rangle\right] .
$$

As the same argument applies to both terms of the symmetric product $q_{i}(z+x) q_{j}(z)$. $q_{i}(0) q_{j}(y)=\frac{1}{2}\left(q_{i}(z+x) q_{j}(z) q_{i}(0) q_{j}(y)+q_{i}(0) q_{j}(y) q_{i}(z+x) q_{j}(z)\right)$, let me just consider the first term. This can be written as

$$
\begin{aligned}
& \int_{-n}^{n} \mathrm{~d} x \int_{-n}^{n} \mathrm{~d} y \int \mathrm{d} z\left[\left\langle q_{i}(z+x) q_{j}(z) q_{i}(0) q_{j}(y)\right\rangle-\left\langle q_{i}(z+x) q_{j}(z)\right\rangle\left\langle q_{i}(0) q_{j}(y)\right\rangle\right] \\
& =\int_{-n}^{n} \mathrm{~d} x \int_{-n}^{n} \mathrm{~d} y \int \mathrm{d} z\left[\left\langle q_{i}(z+x) q_{j}(z) q_{i}(0) q_{j}(y)\right\rangle^{\mathrm{c}}\right. \\
& \left.\quad+\left\langle q_{i}(z+x) q_{i}(0)\right\rangle^{\mathrm{c}}\left\langle q_{j}(z) q_{j}(y)\right\rangle^{\mathrm{c}}+\left\langle q_{i}(z+x) q_{j}(y)\right\rangle^{\mathrm{c}}\left\langle q_{j}(z) q_{i}(0)\right\rangle^{\mathrm{c}}\right] .
\end{aligned}
$$

In (109), the first term in the square brackets on the right-hand side gives under integration a finite value as $n \rightarrow \infty$ by clustering of the four-point function.

For the second term, a change of variable gives

$$
\begin{aligned}
& \int_{-n}^{n} \mathrm{~d} x \int_{-n}^{n} \mathrm{~d} y \int \mathrm{d} z\left\langle q_{i}(z+x) q_{i}(0)\right\rangle^{\mathrm{c}}\left\langle q_{j}(z) q_{j}(y)\right\rangle^{\mathrm{c}} \\
& \quad=\int \mathrm{d} x \mathrm{~d} y(2 n-|x-y|) \Theta(2 n-|x-y|)\left\langle q_{i}(x) q_{i}(0)\right\rangle^{\mathrm{c}}\left\langle q_{j}(y) q_{j}(0)\right\rangle^{\mathrm{c}}
\end{aligned}
$$

where $\Theta(\cdots)$ is the step function. By convergence of the integral of $\left\langle q_{i}(x) q_{i}(0)\right\rangle^{\mathrm{c}}\left\langle q_{j}(y) q_{j}(0)\right\rangle^{\mathrm{c}}$ over $(x, y) \in \mathbb{R}^{2}$, we have

$$
\lim _{n \rightarrow \infty} \int \mathrm{d} x \mathrm{~d} y \Theta(2 n-|x-y|)\left\langle q_{i}(x) q_{i}(0)\right\rangle^{\mathrm{c}}\left\langle q_{j}(y) q_{j}(0)\right\rangle^{\mathrm{c}}=\left\|q_{i}\right\|_{\mathcal{H}^{\prime}}^{2}\left\|q_{j}\right\|_{\mathcal{H}^{\prime}}^{2}
$$

Therefore, on the right-hand side of (110), the term in the parenthesis proportional to $2 n$ gives the contribution

$$
2 n\left\|q_{i}\right\|_{\mathscr{H}^{\prime}}^{2}\left\|q_{j}\right\|_{\mathcal{H}^{\prime}}^{2}
$$

On the other hand, we bound, according to (99) (the case $r=2$, with $d>1$ )

$$
\begin{aligned}
& \left|\int \mathrm{d} x \mathrm{~d} y\right| x-y\left|\Theta(2 n-|x-y|)\left\langle q_{i}(x) q_{i}(0)\right\rangle^{\mathrm{c}}\left\langle q_{j}(y) q_{j}(0)\right\rangle^{\mathrm{c}}\right| \\
& \quad \leq c^{2} \int \mathrm{d} x \mathrm{~d} y|x-y| \Theta(2 n-|x-y|)(b+|x|)^{-d}(b+|y|)^{-d} .
\end{aligned}
$$

We may estimate, say for $z>0$, 


$$
\begin{aligned}
& \int \mathrm{d} x(b+|x|)^{-d}(b+|z+x|)^{-d} \\
& =\left[\int_{-\infty}^{-z / 2}+\int_{-z / 2}^{\infty}\right] \mathrm{d} x(b+|x|)^{-d}(b+|z+x|)^{-d} \\
& \leq \int_{-\infty}^{-z / 2} \mathrm{~d} x(b+z / 2)^{-d}(b+|z+x|)^{-d}+\int_{-z / 2}^{\infty} \mathrm{d} x(b+|x|)^{-d}(b+z / 2)^{-d} \\
& =\frac{4 b^{1-d}(b+z / 2)^{-d}-2(b+z / 2)^{1-2 d}}{d-1} \leq \frac{4 b^{1-d}(b+z / 2)^{-d}}{d-1} .
\end{aligned}
$$

Therefore, the right-hand side of (113) is bounded as

$$
\leq c^{2} \int_{-2 n}^{2 n} \mathrm{~d} z|z| \frac{4 b^{1-d}(b+|z| / 2)^{-d}}{d-1} .
$$

This converges as $n \rightarrow \infty$ if $d>2$, and diverges proportionally to $n^{2-d}$ is $d<2$. Since $d>1$, the divergence as $n^{2-d}$ is sub-dominant compared to $n$, hence in all cases the part (112) dominates and we have

$$
\lim _{n \rightarrow \infty}(2 n)^{-1} \int_{-n}^{n} \mathrm{~d} x \int_{-n}^{n} \mathrm{~d} y \int \mathrm{d} z\left\langle q_{i}(z+x) q_{i}(0)\right\rangle^{\mathrm{c}}\left\langle q_{j}(z) q_{j}(y)\right\rangle^{\mathrm{c}}=\left\|q_{i}\right\|_{\mathscr{H}^{\prime}}^{2}\left\|q_{j}\right\|_{\mathscr{H}^{\prime}}^{2}
$$

The third term in the square brackets on the right-hand side in (109) has the same structure as the second term by a simple change of the $z$ variable. Hence the same derivation can be applied. In the quantum case, we need to be careful with the ordering of the observables in order to identify the integrated two-point correlation functions with inner products in $\mathcal{H}^{\prime}$. By our assumption that the state is invariant under the actions of $Q_{i}$, we have $\left\langle Q_{i} q_{j}(0)\right\rangle^{\mathrm{c}}=$ $\left\langle q_{j}(0) Q_{i}\right\rangle^{\mathrm{c}}$, so that the ordering does not matter. This could also have been obtained by the assumption that all densities $q_{i}$ 's are conserved under all flows generated by $Q_{\ell}$ 's, with $\mathrm{i}\left[Q_{\ell}, q_{i}(x)\right]+\partial_{x} j_{i}^{(\ell)}(x)=0$.

Overall, we obtain

$$
\lim _{n \rightarrow \infty} \frac{\left\|Q_{i j ; n}\right\|_{\mathscr{H}^{\prime}}^{2}}{2 n}=\left\|q_{i}\right\|_{\mathscr{H}^{\prime}}^{2}\left\|q_{j}\right\|_{\mathcal{H}^{\prime}}^{2}+\left\langle q_{i}, q_{j}\right\rangle_{\mathcal{H}^{\prime}}^{2}
$$

This is linear extensivity for $\mathcal{H}^{\prime}$, where we may take any

$$
\gamma>2\left(\left\|q_{i}\right\|_{\mathscr{H}^{\prime}}^{2}\left\|q_{j}\right\|_{\mathscr{H}^{\prime}}^{2}+\left\langle q_{i}, q_{j}\right\rangle_{\mathscr{H}^{\prime}}^{2}\right) .
$$

As for Point $2^{\prime}$, we must show that $\lim _{n \rightarrow \infty}\left\langle Q_{i j ; n}, a\right\rangle_{\mathcal{H}^{\prime}}$ exists for all $a \in \mathcal{V}$. Using 3 -point clustering, this is immediate,

$$
\begin{aligned}
Q_{i j}^{\prime \prime}(a)=\lim _{n \rightarrow \infty}\left\langle Q_{i j ; n}, a\right\rangle_{\mathcal{H}^{\prime}} & =\int \mathrm{d} x\left\langle q_{i}(x) q_{j},\left(1-\mathbb{P}_{\tau}\right) a\right\rangle_{\mathcal{H}^{\prime}} \\
& =\left\langle Q_{i} Q_{j} \cdot\left(1-\mathbb{P}_{\tau}\right) a\right\rangle^{\mathrm{c}} . \\
& =\left\langle Q_{i} Q_{j}\left(1-\mathbb{P}_{\tau}\right) a\right\rangle^{\mathrm{c}} .
\end{aligned}
$$

where in the last line, I use the invariance of the state under the actions of $Q_{i}$ and $Q_{j}$. Note that this also implies that $Q_{i j}^{\prime \prime}$ is invariant under all higher flows $\tau_{s}^{(\ell)}$ with $\ell>2$ (if any), as follows from the associated conservation laws and three-point clustering:

$$
Q_{i j}^{\prime \prime}\left(\tau_{s}^{(\ell)} a\right)=Q_{i j}^{\prime \prime}(a)
$$


Finally, for Point $3^{\prime}$, we use the fact that $Q_{i j ; n}$ is itself a "conserved quantity", in the following sense: From the conservation laws (48), and the fact that $\partial_{t} \tau_{t} \mathbb{P}_{\tau} a=\partial_{t} \mathbb{P}_{\tau} a=0$ for any $a$, we find

$$
\left.\partial_{t}\left\langle\tau_{t} Q_{i j ; n}, a\right\rangle_{\mathcal{H}^{\prime}}\right|_{t=0}=\left\langle j_{i}(-n) q_{j}-j_{i}(n) q_{j}-q_{i}(-n) j_{j}+q_{i}(n) j_{j}, a\right\rangle_{\mathcal{H}^{\prime}} .
$$

Therefore, by time-translation invariance of the inner product, and integrating,

$$
\left.\left\langle Q_{i j ; n}, \tau_{s_{1}} a-\tau_{s_{2}} a\right)\right\rangle_{\mathcal{H}^{\prime}}=\int_{s_{1}}^{s_{2}} \mathrm{~d} t\left\langle j_{i}(-n) q_{j}-j_{i}(n) q_{j}-q_{i}(-n) j_{j}+q_{i}(n) j_{j}, \tau_{t} a\right\rangle_{\mathcal{H}^{\prime}}
$$

I now show that if both $s_{1}, s_{2}$ lie in the open interval $(-n /(2 v), n /(2 v))$, then this vanishes as $n \rightarrow \infty$, giving Point $3^{\prime}$ with any $0<k<1 /(2 v)$. Indeed, intuitively, in this case the observable $\tau_{t} a$ in (122) "covers" a region of length strictly less than $n$, and in a connected three-point function with, for instance, $j_{i}(x+n) q_{j}(x)$ (both at time 0 ), for every $x$, at least one of $j_{i}(x+n)$ or $q_{j}(x)$ lies outside of this interval ${ }^{4}$.

More precisely, we can use the condition (99) for 3-point functions in order to show that each term in the inner product on the right-hand side of (122) gives a vanishing contribution as $n \rightarrow \infty$. Consider a term of the form $\int_{s_{1}}^{s_{2}} \mathrm{~d} t\left\langle b(n) c, \tau_{t} a\right\rangle_{\mathcal{H}^{\prime}}$ with $\langle b\rangle=\langle c\rangle=0$. This is bounded as

$$
\begin{aligned}
\left|\int_{s_{1}}^{s_{2}} \mathrm{~d} t\left\langle\mathcal{b}(n) c, \tau_{t} a\right\rangle_{\mathcal{H}^{\prime}}\right| & \leq \int_{s_{1}}^{s_{2}} \mathrm{~d} t \int \mathrm{d} x\left|\langle a(x, t) \cdot b(n, 0) c(0,0)\rangle^{\mathrm{c}}\right| \\
& =n^{2} \int_{-k}^{k} \mathrm{~d} t \int \mathrm{d} x\left|\langle a(n x, n t) \cdot b(n, 0) c(0,0)\rangle^{\mathrm{c}}\right|
\end{aligned}
$$

where it is sufficient to consider $s_{1}=-n k, s_{2}=n k$ with $k>0$. For now I am not imposing any additional condition on $k$. Using (99) for the 3-point function on the last line, three values are to be compared: $z_{12}=n, z_{13}=n(|1-x|-v|t|), z_{23}=n(|x|-v|t|)$, with $|t|<k$. I analyse the double integral in (123) by looking at two distinct regions:

$$
R_{1}=\{(x, t):|x-1 / 2|>3 / 2+k v,|t|<k\}, \quad R_{2}=\left\{(x, t):|x-1 / 2| \leq 3 / 2+k v,|t| \leq k^{\prime}\right\}
$$

for some $k^{\prime}<1 /(2 v)$. Doing it this way is useful for the proofs of other theorems below. For these to cover the full integration region, the condition $k \leq k^{\prime}$ is then imposed.

On the one hand, in $R_{1}$, the quantity $z=\min \left\{z_{13}, z_{23}\right\}$ is the maximal value, and is positive. Thus we can use the bound $f(z)=c(b+z)^{-d}$, and we bound the integral over this region on the right-hand side of (123) as

$$
\begin{aligned}
& c n^{2} \int_{-k}^{k} \mathrm{~d} t \int_{|x-1 / 2|>3 / 2+k v} \mathrm{~d} x[b+n(\min \{|1-x|,|x|\}-v|t|)]^{-d} \\
& =\frac{c}{(d-1)(d-2) v}\left[(b+n(1+2 k v))^{2-d}-(b+n)^{2-d}\right]
\end{aligned}
$$

where the $x$ integral is convergent as $d>1$. Since in fact $d>2$, this result vanishes in the limit $n \rightarrow \infty$.

On the other hand, in $R_{2}$, the lower bound of the minimum of $|1-x|-v|t|$ and $|x|-v|t|$ is positive: the former is positively lower bounded on $x \leq 1 / 2$, the latter on $x \geq 1 / 2$. As $z_{12}$

4 To be precise, the boundary $1 /(2 v)$ can also be included, as under integration its nonvanishing contribution has zero measure; but this does not affect the final result. 
is positive, we find that $z=\max _{i}\left\{\min _{j}\left\{z_{i j}\right\}\right\}$ is positive, and we can again use the bound $f(z)=c(b+z)^{-d}$. The minimal value of $z$ for $|x-1 / 2| \leq 3 / 2+k v$ and $|t| \leq k^{\prime}$ is obtained at $x=1 / 2$ and $t= \pm k^{\prime}$ and given by $n\left(1 / 2-k^{\prime} v\right)$. Taking this minimum value, we bound the integral over this region on the right-hand side of (123) as

$c n^{2} \int_{-k^{\prime}}^{k^{\prime}} \mathrm{d} t \int_{|x-1 / 2| \leq 3 / 2+k v} \mathrm{~d} x\left[b+n\left(1 / 2-k^{\prime} v\right)\right]^{-d}=(3 / 2+k v) 2 k c n^{2}\left[b+n\left(1 / 2-k^{\prime} v\right)\right]^{-d}$.

Since $d>2$, this result vanishes in the limit $n \rightarrow \infty$.

With $k \leq k^{\prime}$, this cover the full integration region in (123), and we have shown that it vanishes as $n \rightarrow \infty$. This therefore imposes $k \leq 1 /(2 v)$.

Therefore, the resulting map (119) is a quadratically extensive charge for $\mathcal{H}$, with $\mathcal{V} \subset$ $D^{\prime \prime}(Q)$. Using (118) and Theorem 4.1, and optimising the lower bound, we have shown the theorem.

\subsection{Diffusion from Hydrodynamic Correlations}

The Lieb-Robinson-type bound (99) is rigorously established at least for $r=2$ in quantum chains, but it is not state dependent, and not optimal. In general, one expects clustering (99) to hold for velocities $v$ smaller than the Lieb-Robinson velocity $v_{\mathrm{LR}}$, up to an optimal value that is state-dependent; this value can be further optimised for certain observables. A natural guess is that $v$ in (99) and (103) can be set to the largest hydrodynamic velocity (the largest propagation velocity of normal modes, see Appendix 2). In fact, one can derive stronger clustering statements by applying simple hydrodynamic-type linear response arguments, of the type used in [42]. In this section, I propose a general clustering statement based on the results of such linear response arguments, from which I derive stronger bounds on diagonal Onsager matrix elements.

It is sufficient to concentrate on three-point functions. Hydrodynamic principles give results for Euler-scale correlation functions $\langle\cdots\rangle^{\text {eul }}$, where space and time coordinates have been sent to infinity in fixed proportions; for discussions of the Euler scaling limit, see [1,42]. In Appendix 5, I obtain two results concerning Euler-scale three-point functions of the type $\left\langle\left(1-\mathbb{P}_{\tau}\right) a(x, t) \mathcal{b}(y, 0) c(z, 0)\right\rangle^{\text {eul }}:(1)$ in such a three-point function, local observables $\mathfrak{b}$ and $c$ project onto conserved densities, Eq. (187); and (2) conserved densities propagate ballistically, Eq. (189). These statements are obtained using linear response arguments in the classical context, but are expected to hold in the quantum context as well.

The latter statement, expressed in terms of normal modes, takes the form

$$
\begin{aligned}
& \left\langle\left(1-\mathbb{P}_{\tau}\right) a(x, t) \cdot q_{I}(y, 0) q_{J}(z, 0)\right\rangle^{\mathrm{eul}} \\
& \quad=\left\langle Q_{I} Q_{J}\left(1-\mathbb{P}_{\tau}\right) a\right\rangle^{\mathrm{c}} \delta\left(x-y-v_{I}^{\mathrm{eff}} t\right) \delta\left(x-z-v_{J}^{\mathrm{eff}} t\right) .
\end{aligned}
$$

As the currents of normal modes project diagonally, $\mathbb{P}_{\tau} j_{I}=v_{I}^{\text {eff }} q_{I}$, combining with the former statement, a similar formula holds with currents instead of densities, for instance

$$
\begin{aligned}
& \left\langle\left(1-\mathbb{P}_{\tau}\right) a(x, t) \cdot j_{I}(y, 0) q_{J}(z, 0)\right\rangle^{\mathrm{eul}} \\
& \quad=v_{I}^{\mathrm{eff}}\left\langle Q_{I} Q_{J}\left(1-\mathbb{P}_{\tau}\right) a\right\rangle^{\mathrm{c}} \delta\left(x-y-v_{I}^{\mathrm{eff}} t\right) \delta\left(x-z-v_{J}^{\mathrm{eff}} t\right) .
\end{aligned}
$$

In (127) and (128), the delta-functions arise from the Euler scaling; microscopic threepoint functions are expected to have support around these ballistic trajectories with power-law decay. The clustering assumption I propose makes this more precise. As I do not know of 
rigorous results in this direction for any specific class of models, I will make the assumption as general as possible, in accordance with the expected phenomenology and the above formulae.

Thus, in addition to (99), I assume that, for all $\epsilon>0$, and for $b, c$ being densities or currents $q, j$, connected three-point functions $\left\langle\left(1-\mathbb{P}_{\tau}\right) a(x, t) \cdot b_{I_{1}}\left(y_{1}, 0\right) c_{I_{2}}\left(y_{2}, 0\right)\right\rangle^{\mathrm{c}}$ decay fast enough whenever $\left|x-y_{1}-v_{I_{1}}^{\text {eff }} t\right|-\epsilon|t| \gg 0$, or $\left|x-y_{2}-v_{I_{2}}^{\text {eff }} t\right|-\epsilon|t| \gg 0$; that is, whenever $(x, t)$ lies away from either of the ballistic trajectories emanating from $\left(y_{1}, 0\right)$ and $\left(y_{2}, 0\right)$. More precisely, given $a \in \mathcal{V}$, normal-mode indices $I_{1}, I_{2}$, and $\epsilon>0$, there exist a real number $d>2$, and a function $f(z, t)>0(z>0, t \in \mathbb{R})$ that is $(-d)$-homogeneous, $f(n z, n t)=n^{-d} f(z, t) \forall n>0$, monotonically decreasing in $z, f(z, t)<f\left(z^{\prime}, t\right)$ if $z>z^{\prime}$, and uniformly bounded on $\{(z, t): t \in T\}$ for any $z \neq 0$ and compact subset $T \not \supset\{0\}$, such that

$$
\begin{array}{r}
\left|\left\langle\left(1-\mathbb{P}_{\tau}\right) a(x, t) \cdot b_{I_{1}}\left(y_{1}, 0\right) c_{I_{2}}\left(y_{2}, 0\right)\right\rangle^{\mathrm{c}}\right| \leq f\left(\max _{i}\left\{z_{i}\right\}, t\right) \\
\text { if } \max _{i}\left\{z_{i}\right\}>0, \quad z_{i}=\left|x-y_{i}-v_{I_{i}}^{\mathrm{eff}} t\right|-\epsilon|t| .
\end{array}
$$

Again, the somewhat complicated specifications of the function $f(z, t)$ are for generality of the theorem. For instance, the functions $f(z, t)=z^{-d+v}|t|^{-v}$ for $v<d$ would do, as well as any linear combination thereof with positive coefficients. Pictorially, this condition can be represented by Fig. 1, with a sub-linear long-time scaling $g(t) \ll t$ and conditions on some of the observables being normal modes (as above).

This gives rise to a strengthening of Theorem 5.1, in particular proving that, if certain conditions are met, there must be superdiffusion:

Theorem 5.2 Assume that (99) holds. Let $a \in \mathcal{V}$, and let $I, J$ be normal-mode indices, and assume that (129) hold for this a and $I_{1}=I, I_{2}=J$. If $v_{I}^{\mathrm{eff}} \neq v_{J}^{\mathrm{eff}}$, then

$$
\mathfrak{L}_{a, a}^{-} \geq \frac{\left|\left\langle Q_{I} Q_{J}\left(1-\mathbb{P}_{\tau}\right) a\right\rangle^{\mathrm{c}}\right|^{2}}{\left|v_{I}^{\mathrm{eff}}-v_{J}^{\mathrm{eff}}\right|} .
$$

If $v_{I}^{\mathrm{eff}}=v_{J}^{\mathrm{eff}}$ (including the case $I=J$ ) and

$$
\left\langle Q_{I} Q_{J}\left(1-\mathbb{P}_{\tau}\right) a\right\rangle^{\mathrm{c}} \neq 0,
$$

then $\mathfrak{L}_{a, a}^{-}=\infty$. In particular, if (131) holds for a current observable $a=j_{i}$ and some $I=J$, then the associated lower Onsager matrix element is infinite, $\mathfrak{L}_{i i}^{-}=\infty$ (hence there is superdiffusion).

Proof The proof of Points $1^{\prime}, 2^{\prime}$ and $3^{\prime}$ that $Q=\left(Q_{I J ; n}: n \in \mathbb{N}\right)$ gives a linearly extensive charge in Sect. 5.2 still holds true. However, with (129), we can strengthen the analysis of (122) for Point $3^{\prime}$, and thus optimise the range of values of $k$. We look for $k>0$ such that $\lim _{n \rightarrow \infty} \sup _{s, t \in[-k n, k n]}\left|\left\langle Q_{I J ; n}, \tau_{s} a-\tau_{t} a\right\rangle_{\mathcal{H}}\right|=0$. Because $Q_{I J ; n}=\left(1-\mathbb{P}_{\tau}\right) Q_{I J ; n}$, we can make the replacement $a \rightarrow\left(1-\mathbb{P}_{\tau}\right) a$ in (121) and (122). Again, we bound a typical term as

$$
\begin{aligned}
& \left|\int_{s_{1}}^{s_{2}} \mathrm{~d} t\left\langle b_{I_{1}}(n) c_{I_{2}},\left(1-\mathbb{P}_{\tau}\right) \tau_{t} a\right\rangle_{\mathcal{H}^{\prime}}\right| \\
& \quad \leq n^{2} \int_{-k}^{k} \mathrm{~d} t \int \mathrm{d} x\left|\left\langle\left(1-\mathbb{P}_{\tau}\right) a(n x, n t) \cdot b_{I_{1}}(n, 0) c_{I_{2}}(0,0)\right\rangle^{\mathrm{c}}\right|
\end{aligned}
$$

with $s_{1}=-n k, s_{2}=n k$ for some $k>0$ to be determined. The paragraph just after (123) shows that, using the bound (99) for the 3-point function on the right-hand side, the 
contributions in the regions $R_{1}$ and $R_{2}$ defined by (124) vanish as $n \rightarrow \infty$ for any $k^{\prime}<1 /(2 v)$, where $v$ is the velocity from the assumption (99). Therefore, it is sufficient to concentrate on the rest,

$$
R_{3}=\left\{(x, t):|x-1 / 2| \leq 3 / 2+k v, k^{\prime}<|t|<k\right\} .
$$

In order to use the bound in (129), we must guarantee that, in this region, $\max _{i}\left\{z_{i}\right\}$ has a positive lower bound, where $z_{1}=n\left(\left|x-1+v_{1} t\right|-\epsilon|t|\right)$ and $z_{2}=n\left(\left|x+v_{2} t\right|-\epsilon|t|\right)$ (with $v_{i}=v_{I_{i}}^{\text {eff }}$ ). Let $y=x-1 / 2+\left(v_{2}+v_{1}\right) t / 2$ and $y_{t}=1 / 2+\left(v_{2}-v_{1}\right) t / 2$. Then $z_{1} / n=\left|y-y_{t}\right|-\epsilon|t|$ and $z_{2} / n=\left|y+y_{t}\right|-\epsilon|t|$. Note that $y_{0}>0$. For every $y_{t}>0$, the maximum $z=\max _{i}\left\{z_{i}\right\}$ is $z_{2}\left(z_{1}\right)$ if $y>0(y<0)$. Over $y$, the minimal value of $z$ is at $y=0$, and is $n\left(y_{t}-\epsilon|t|\right)$. Thus if $k$ is chosen such that $y_{t}>\epsilon|t|$ for all $|t| \leq k$, this minimal value is positively bounded on the time integration region. Then, with $m=\inf _{|t|<k}\left(y_{t}-\epsilon|t|\right)$ and by monotonicity and $(-d)$-homogeneity of $f(z, t)$, we have

$$
\begin{aligned}
& n^{2} \int_{R_{3}} \mathrm{~d} t \mathrm{~d} x\left|\left\langle\left(1-\mathbb{P}_{\tau}\right) a(n x, n t) \cdot b_{I_{1}}(n, 0) \mathcal{c}_{I_{2}}(0,0)\right\rangle^{\mathrm{c}}\right| \\
& \leq n^{2} \int_{k^{\prime}<|t|<k} \mathrm{~d} t \int_{|x-1 / 2| \leq 3 / 2+v k} \mathrm{~d} x f(n m, n t) \\
& =n^{2-d} \int_{k^{\prime}<|t|<k} \mathrm{~d} t \int_{|x-1 / 2| \leq 3 / 2+v k} \mathrm{~d} x f(m, t) .
\end{aligned}
$$

As the time integration region is finite and excludes a neighbourhood of $t=0$, the function $f(m, t)$ is uniformly bounded on this region. Since $d>2$, the result vanishes as $n \rightarrow \infty$. Considering terms where $I_{1}$ and $I_{2}$ are exchanged, the conditions $y_{t}>\epsilon|t|$ are all satisfied if $|t|<1 /\left(\left|v_{1}-v_{2}\right|+2 \epsilon\right)$. As a consequence one can choose any $0<k<1 /\left(\left|v_{I}^{\text {eff }}-v_{J}^{\text {eff }}\right|+2 \epsilon\right)$ for $\lim _{n \rightarrow \infty} \sup _{s, t \in[-k n, k n]}\left|\left\langle Q_{I J ; n}, \tau_{s} a-\tau_{t} a\right\rangle_{\mathcal{H}}\right|=0$. Thus, using $\left\|q_{I}\right\|_{\mathscr{H}^{\prime}}^{2}=\left\|q_{J}\right\|_{\mathscr{H}^{\prime}}^{2}=1$ and $\left\langle q_{I}, q_{J}\right\rangle_{\mathcal{H}^{\prime}}=0$ and taking $\epsilon>0$ arbitrarily small, Theorem 4.1 implies the theorem.

Remark 5.3 The assumption (129) should be interpreted in view of the discussion of superdiffusion in Sect. 3.5 and Appendix 4. In the normal superdiffusion case, exponential decay, or decay with high enough power law, away from the ballistic trajectory can be assumed, and thus the assumption is expected to hold with $d$ arbitrarily large. In the fat superdiffusion case, by (179) one may expect weaker power-law clustering in the region around the ballistic trajectory. For two-point functions of conserved densities one has $O\left(|t|\left|x-y_{i}-v_{I_{i}}^{\mathrm{eff}} t\right|^{-1-1 / \alpha}\right)$, from (177) with (179). For two-point functions involving currents, powers of $|t|$ decrease and of $\left|x-y_{i}-v_{I_{i}}^{\text {eff }} t\right|$ increase in equal amount. Higher-point functions are subject to different power laws, and Euler scaling predicts one less power for every observable [42]. Thus, one might expect $O\left(|t|^{-v}\left|x-y_{i}-v_{i} t\right|^{\nu-1 / \alpha-1}\right)$ for values $v<2$; which is indeed in agreement with (129) for $\alpha \in(1 / 2,1)$, where one finds $d=1 / \alpha+1>2$. A more in-depth analysis of three-point function clustering would be useful.

\subsection{Superdiffusion}

I now propose a natural clustering property, inferred from the superdiffusion phenomenology, under which the conditions and related superdiffusion exponents found from NFH naturally emerge. This will show that these characteristics of superdiffusion, usually explained using $\mathrm{NFH}$, actually arise without the need for the hydrodynamic equation, mode-coupling theory or the addition of noise. 
Note that Theorem 5.2 implies that there is superdiffusion for the normal mode $K$ if the coupling $\left\langle Q_{I}^{2}\left(1-\mathbb{P}_{\tau}\right) j_{K}\right\rangle^{\mathrm{c}} \neq 0$ for some $I$. This condition is exactly that found from NFH [2] for superdiffusive behaviours of normal modes: these couplings are the " $G$-couplings" introduced in this context. Here, it is seen to hold as well in the quantum context.

In NFH, a variety of superdiffusion exponents and scaling functions are found, depending on which $G$-coupling(s) is (are) nonzero. In particular, it is found, in the classical context, that if the fully diagonal coupling is nonzero, $\left\langle Q_{I}^{2}\left(1-\mathbb{P}_{\tau}\right) j_{I}\right\rangle^{\mathrm{c}} \neq 0$, then superdiffusion is within the Kardar-Parisi-Zhang (KPZ) universality class, with exponent $\alpha_{I}=2 / 3$. This is the case of sound modes in generic anharmonic chains and other momentum conserving one-dimensional systems [2]. Further, using mode-coupling theory, it is found that if the fully diagonal coupling is zero, $\left\langle Q_{K}^{2}\left(1-\mathbb{P}_{\tau}\right) j_{K}\right\rangle^{\mathrm{c}}=0$, but a partially diagonal coupling is nonzero, $\left\langle Q_{I}^{2}\left(1-\mathbb{P}_{\tau}\right) j_{K}\right\rangle^{\mathrm{c}} \neq 0$ for some KPZ-superdiffusive mode $I$ and some $K \neq I$, then superdiffusion for mode $K$ is controlled by the Lévy $\left(\alpha_{K}^{-1}\right)$-stable distribution, with exponent $\alpha_{K}=3 / 5$. This is the case of the heat mode in generic anharmonic chains [2]. More generally, it is argued that if the nonzero couplings are a single fully diagonal one, $\left\langle Q_{I}^{2}\left(1-\mathbb{P}_{\tau}\right) j_{I}\right\rangle^{\mathrm{c}} \neq 0$, at the root of a sequence of nonzero partially diagonal ones, $\left\langle Q_{K}^{2}\left(1-\mathbb{P}_{\tau}\right) j_{K+1}\right\rangle^{\mathrm{c}} \neq 0$, $K=I, I+1, \ldots, K_{\max }-1$, then the superdiffusion scaling functions are Levy distribution with exponents obtained from the Fibonacci sequence, see [5] for details.

Here I obtain a set of relations between conditions of nonzero $G$-couplings and bounds superdiffusion exponents that, in particular, exactly reproduce the above structure, in both the classical and quantum contexts.

Recall the result (127), which follows from a linear response analysis. With $I=J$, we have co-propagation of the normal modes, and this is at the basis of the divergence of the Onsager matrix element in Theorem 5.2 (as $v_{I}^{\text {eff }}=v_{J}^{\text {eff }}$ ). However, as mentioned, the actual propagation of normal modes is expected to lead, at least from the viewpoint of correlation functions, to a power-law extension around the ballistic trajectory. That is, a strong enough clustering property should be applicable in the full region away from this power-law expanding domain. Considering this full region, one can get a more precise characterisation of superdiffusion.

According to (177), one would expect this domain to expand with the superdiffusion exponent $\alpha_{I}$ for mode $I$. Thus, I assume the following. Let $a \in \mathcal{V}$, let $b, c$ be densities or currents $q, j$, let $I$ be a normal-mode index, with superdiffusive exponent $\alpha=\alpha_{I}$ in Eq. (177), and let

$$
u>\alpha_{I}+1 \in(1,2) .
$$

Then there exist real numbers $p \geq 0$ and $d>2+(2-u) p$, and a function $f(z, t)>0$ $(z>0, t \in \mathbb{R})$ that is $(-d)$-homogeneous, $f(n z, n t)=n^{-d} f(z, t) \forall n>0$, monotonically decreasing in $z, f(z, t)<f\left(z^{\prime}, t\right)$ if $z>z^{\prime}$, and such that $|t|^{1+p} f(z, t)$ is uniformly bounded on $\{(z, t): t \in T\}$ for any $z \neq 0$ and compact subset $T$, such that:

$$
\begin{array}{r}
\left|\left\langle\left(1-\mathbb{P}_{\tau}\right) a(x, t) \cdot b_{I}\left(y_{1}, 0\right) c_{I}\left(y_{2}, 0\right)\right\rangle^{\mathrm{c}}\right| \leq f\left(\max _{i}\left\{z_{i}\right\}, t\right) \\
\text { if } \max _{i}\left\{z_{i}\right\}>0 \quad\left(z_{i}=\left|x-y_{i}-v_{I}^{\mathrm{eff}} t\right|-|\kappa t|^{u-1}\right) .
\end{array}
$$

The main difference with (129) is that the cone, which was controlled by $\epsilon>0$, around the ballistic trajectory beyond which there is clustering, is now replaced by the power-law expanding region controlled by the strength $\kappa$ and the power $u-1$ (here I use my convention in Sect. 3.5 with $u \in(1,2))$. Another difference lies in the function $f(z, t)$ controlling the decay beyond this power-law region. Near $t=0$ it is constrained to diverge at most as a power law, 
and the bound on $d$ is, generically, greater than 2. Again, the functions $f(z, t)=z^{-d+v}|t|^{-v}$ for $v<d$ would do, as well as linear combinations with positive coefficients. In these cases, one takes $p=\max \{0, v-1\}$, and a calculation shows that the values $d>u /(u-1)$ always satisfy the required bound. See Remarks (5.3) and (5.4) for a connection with the superdiffusive phenomenology. Pictorially, this condition can be represented by Fig. 1, with a spreading $g(t)$ as explained in Sect. 2 (and the correspondence $1 / z=\alpha_{I}$ ).

With this, I show that the sequence $Q_{I I ; n}$, Eq. (98), is a $u$-extensive sequence on the observable $a$. The corresponding $u$-extensive charge acts again as $Q^{(u)}(a)=\left\langle Q_{I}^{2}\left(1-\mathbb{P}_{\tau}\right) a\right\rangle^{\mathrm{c}}$. Theorem 4.2 then shows that if this is nonzero for some current $a=j_{K}$ with normal (resp. fat) superdiffusion exponent $\alpha_{K}$, then $\alpha_{K} \geq(3-u) / 2$ (resp. $\alpha_{K} \geq 1 / u$ ). The nontrivial results in the following theorem then arise by connecting the conditions on $u$ from, on the one hand, the superdiffusion exponent $\alpha_{I}$ controlling the extent of the domain beyond which the $I^{\text {th }}$ mode clusters, Eq. (135), and, on the other hand, Theorem 4.2, which relies on the superdiffusion class of the current $j_{K}$-the exponent necessary for convergence of spacetime integrated connected current-current correlation functions. Note that, although there is no full rigour here, there is no arbitrariness in the conditions imposed: Eq. (135) expresses the superdiffusive behaviour with exponent $\alpha_{I}$ around the ballistic trajectories as applied to three-point functions; and Theorem 4.2 follows from the the superdiffusive behaviour of twopoint functions. In particular, taking $I=K$, this leads to specific numerical lower bounds for $\alpha_{I}$.

Theorem 5.3 Assume the clustering properties (99). Assume that the additional clustering condition (136) with (135) holds for some mode I, and, in Point A (resp. B) below, for $a=j_{I}$ (resp. $a=j_{K}$ ). Then

A. If $\left\langle Q_{I}^{2}\left(1-\mathbb{P}_{\tau}\right) j_{I}\right\rangle^{\mathrm{c}} \neq 0$, and if $j_{I}$ has normal (resp. fat) superdiffusion exponent $\alpha_{I}$, Eq. (78) (resp. Eq. (79)), then

$$
\alpha_{I} \geq \frac{2}{3}\left(\operatorname{resp} \cdot \alpha_{I} \geq \frac{2}{\sqrt{5}+1}\right) .
$$

B. If $\left\langle Q_{I}^{2}\left(1-\mathbb{P}_{\tau}\right) j_{K}\right\rangle^{\mathrm{c}} \neq 0$, and if $j_{K}$ has normal (resp. fat) superdiffusion exponent $\alpha_{K}$, Eq. (78) (resp. Eq. (79)), then

$$
\alpha_{K} \geq 1-\frac{\alpha_{I}}{2} \quad\left(\text { resp. } \alpha_{K} \geq \frac{1}{\alpha_{I}+1}\right) .
$$

Proof I show that the clustering condition (136) with (135) implies that $Q_{I I ; n}$ is a $u$-extensive sequence for the observable $a$, for all $u>\alpha_{I}+1$. For simplicity I assume $v_{I}^{\text {eff }}=0$; the case $v_{I}^{\text {eff }} \neq 0$ is easily dealt with by similar arguments, using an additional shift $x \rightarrow x+v_{I}^{\text {eff }} t$ in the integration variable below. The result will then follow from Theorem 4.2.

Points $1^{\prime}$ and $2^{(u)}$ of Sect. 4.3 with $u \in(1,2)$, are immediate from the proof of Theorem 5.1. For Point $3^{(u)}$, we need to show from (122) that there exists $k>0$ such that

$$
\lim _{n \rightarrow \infty} \sup _{s, t \in\left[-k n^{1 /(u-1)}, k n^{1 /(u-1)}\right]}\left|\left\langle Q_{I I ; n}, \tau_{s} a-\tau_{t} a\right\rangle_{\mathcal{H}^{\prime}}\right|=0 .
$$

Again, we bound a typical term in (122) (under the replacement $a \rightarrow\left(1-\mathbb{P}_{\tau}\right) a$ ) as

$$
\begin{aligned}
& \left|\int_{s_{1}}^{s_{2}} \mathrm{~d} t\left\langle b_{I}(n) c_{I},\left(1-\mathbb{P}_{\tau}\right) \tau_{t} a\right\rangle \mathcal{H}^{\prime}\right| \\
& \quad \leq m^{2} \int_{-k}^{k} \mathrm{~d} t \int \mathrm{d} x\left|\left\langle\left(1-\mathbb{P}_{\tau}\right) a(m x, m t) \cdot b_{I}(n, 0) c_{I}(0,0)\right\rangle^{\mathrm{c}}\right|
\end{aligned}
$$


where $m=n^{1 /(u-1)}(\gg n$ as $n \rightarrow \infty)$ and $s_{1}=-m k, s_{2}=m k$ with $k>0$ to be determined. As in the paragraph just after (123), we may use the bound (99) for the 3-point function on the right-hand side. The regions (124) are now expressed as

$$
\begin{aligned}
& R_{1}=\{(x, t):|x-n /(2 m)|>3 n /(2 m)+k v,|t|<k\} \\
& R_{2}=\left\{(x, t):|x-n /(2 m)| \leq 3 n /(2 m)+k v,|t| \leq n k^{\prime} / m\right\}
\end{aligned}
$$

for some $k^{\prime}<1 /(2 v)$, where $v$ is the velocity of the bound (99). In these regions, the contribution of the integral on the right-hand side of (139) vanishes as $n \rightarrow \infty$. Thus we may restrict to

$$
R_{4}=\left\{(x, t):|x| \leq 1+v k, n k^{\prime} / m<|t|<k\right\} .
$$

We now use (136). With $m^{u-1}=n$, we have $z_{1}=|m x-n|-n|\kappa t|^{u-1}$ and $z_{2}=m|x|-$ $n|\kappa t|^{u-1}$. The minimal value over $x$ of $\max _{i}\left\{z_{i}\right\}$ is obtained at $x=n /(2 m)$ and is $n(1 / 2-$ $\left.|\kappa t|^{u-1}\right)$. This is positively bounded on $t \in[-k, k]$ if $k<\kappa^{-1} 2^{1 /(1-u)}$. Then, with $s=$ $\inf _{|t|<k}\left(\left(1 / 2-|\kappa t|^{u-1}\right)\right.$ and by monotonicity and $(-d)$-homogeneity of $f(z, t)$, we have

$$
\begin{aligned}
& m^{2} \int_{R_{4}} \mathrm{~d} t \mathrm{~d} x\left|\left\langle\left(1-\mathbb{P}_{\tau}\right) a(m x, m t) \cdot b_{I}(n, 0) c_{I}(0,0)\right\rangle^{\mathrm{c}}\right| \\
& \leq m^{2} \int_{n k^{\prime} / m<|t|<k} \mathrm{~d} t \int_{|x| \leq 1+v k} \mathrm{~d} x f(m s, m t) \\
& =m^{2-d} 2(1+v k) \int_{m^{u-2} k^{\prime}<|t|<k} \mathrm{~d} t f(s, t) .
\end{aligned}
$$

Under the conditions on the function $f(z, t)$ expressed above (136), the time integral is finite for $p=0$, or else diverge as $m^{p(2-u)}$. The requirement that $d>2+(2-u) p$ then guarantees that the limit $m \rightarrow \infty$ of the right-hand side of (142) vanishes.

The theorem is proved using Theorem 4.2 and minimising $u$.

The sound modes discussed above have nonzero fully diagonal coupling. As the KPZ scaling function has finite variance, the superdiffusion is normal. The lower bound in Point A, $\alpha_{I} \geq 2 / 3$, is then in agreement with the KPZ exponent $2 / 3$, which saturates it. The heat mode discussed above has zero fully diagonal coupling, but nonzero partially diagonal couplings to the sound modes. As the Levy distribution has infinite variance, superdiffusion is fat in this case. The lower bound in Point B, taking $\alpha_{I}=2 / 3$, is then $\alpha_{K} \geq 3 / 5$. This is again in agreement with the superdiffusion exponent found in this case, the bound being saturated. More generally, taking the sequence of nonzero couplings as discussed above, using the fact that the superdiffusion is fat, and assuming the bound in Point B to be saturated, we have $\alpha_{K+1}=1 /\left(\alpha_{K}+1\right)$, and this reproduces the exponents based on the Fibonacci sequence [5].

Interestingly, we obtain strong statements concerning the relation between exponents and tails of the distribution. For instance, we deduce that if the partially diagonal coupling of mode $K$ with mode $I$ is nonzero, $\left\langle Q_{I}^{2}\left(1-\mathbb{P}_{\tau}\right) j_{K}\right\rangle^{\mathrm{c}} \neq 0$, if mode $I$ is of KPZ type, and if the exponent of mode $K$ is smaller than $2 / 3$, as it is indeed observed to be $(3 / 5<2 / 3)$, then the scaling function of mode $K$ must have divergent variance. This is indeed the case, as it is the Levy distribution. This is a nontrivial consequence on the scaling function, inferred solely from the associated exponent.

Note that one should expect the bounds to be saturated. Indeed, for instance, $Q_{\mathscr{H}^{(u)}}\left(j_{I}\right)$ is finite, and one would expect to be able to bound it as in (95) by a finite upper bound on the optimal $u$; thus $\left\|j_{I}\right\|_{\mathscr{H}^{(u)}}^{-}$should be finite on this optimal $u$. 
It is also possible to argue for the KPZ lower bound in the normal superdiffusion case using factionally extensive charges of the Prosen type, see Appendix 8.

Remark 5.4 Continuing on Remark 5.3, recall that the phenomenological expectation of the behaviour of the three-point function in (136) is as $O\left(|t|^{-v}\left|x-y_{i}-v_{i} t\right|^{v-1 / \alpha_{I}-1}\right)$, for values $v<2$. This is indeed in agreement with the specifications of the clustering requirement (136) for $\alpha_{I} \in(1 / 2,1)$, where one finds $d=1 / \alpha_{I}+1>u /(u-1)$ under the inequality (135).

Remark 5.5 If (136) held for $u=\alpha_{I}+1$, then we could use this value in Theorem 4.2, and apply it in order to obtain a bound on the strength of the superdiffusion spreading $\lambda$ in Eq. (177). However, I do not expect (136) to hold for $u=\alpha_{I}+1$, for any $\kappa$. Indeed, exactly on the curve of the superdiffusion growth, there are corrections and clustering is not uniformly vanishing. One might also hope to weaken Point $3^{(u)}$ in order to include finite corrections, which might be supported in small regions and therefore would give vanishing contributions to $\delta_{n}$ in (91) (here replacing $u$ by $u-1$ ). However, although the nonzero correlations are only "near" the curve of superdiffusion growth, I expect the extent of the spatial region on which they lie to grow with the same power, as $t^{u-1}$. Therefore, the correction term $\delta_{n}$ receives nonvanishing contributions. Hence, the techniques proposed here seem to be too rough to give bounds on the superdiffusion strength.

\section{Additional Remarks}

\subsection{Two-Body Wave Scattering}

Relation (127), which is derived in Appendix 5 and used in Sect. 5.3 in order to justify the clustering assumption used there, allows us to go further and determine the exact element of $\mathscr{H}^{\prime \prime}$ which represent the charge $Q_{I J}^{\prime \prime}$. That is, as per (88), there must exist $w_{I J} \in \mathcal{H}^{\prime \prime}$ such that

$$
Q_{I J}^{\prime \prime}(a)=\left\langle w_{I J}, a\right\rangle_{\mathcal{H}^{\prime \prime}}
$$

I show in Appendix 6, using Euler-scale arguments, that

$$
w_{I J}=\lim _{X \rightarrow \infty} \frac{\left|v_{I}^{\text {eff }}-v_{J}^{\text {eff }}\right|}{2 X} \int_{-X}^{X} \mathrm{~d} x q_{I}(x) q_{J} .
$$

The limit in $X$ is to be taken within $\mathcal{H}^{\prime \prime}$, that is, one evaluates this limit after taking the limit over time in the second equation in (41). The expression within the limit in (144) is a Cauchy sequence within this Hilbert space, and it is the limit that gives an element of $\mathscr{H}^{\prime \prime}$. From this, the overlaps between $w_{I J}$ and $w_{K L}$ are evaluated by combining (143) and (144):

$$
\begin{aligned}
& \left\langle w_{I J}, w_{K L}\right\rangle_{\mathcal{H}^{\prime \prime}} \\
& =Q_{I J}^{\prime \prime}\left(w_{K L}\right) \\
& =\frac{\left|v_{K}^{\text {eff }}-v_{L}^{\text {eff }}\right|}{2 X} \lim _{X \rightarrow \infty} \int_{-X}^{X} \mathrm{~d} x Q_{I J}^{\prime \prime}\left(q_{K}(x) q_{L}\right) \\
& =\frac{\left|v_{K}^{\text {eff }}-v_{L}^{\text {eff }}\right|}{2 X} \lim _{X \rightarrow \infty} \int_{-X}^{X} \mathrm{~d} x\left[\left\langle Q_{I} Q_{J} q_{K}(x) q_{L}\right\rangle^{\mathrm{c}}+\left\langle Q_{I} q_{K}(x)\right\rangle^{\mathrm{c}}\left\langle Q_{J} q_{L}\right\rangle^{\mathrm{c}}\right. \\
& \left.\quad+\left\langle Q_{I} q_{L}(x)\right\rangle^{\mathrm{c}}\left\langle Q_{J} q_{K}(x)\right\rangle^{\mathrm{c}}-\sum_{M}\left\langle Q_{I} Q_{J} q_{M}\right\rangle^{\mathrm{c}}\left\langle Q_{M} q_{K}(x) q_{L}\right\rangle^{\mathrm{c}}\right] .
\end{aligned}
$$


The first three terms inside the bracket are the expression of the three-point connected function in (119) in terms of a four-point connected function. The last term inside the bracket integrates to $\sum_{M}\left\langle Q_{I} Q_{J} q_{M}\right\rangle^{\mathrm{c}}\left\langle Q_{M} Q_{K} q_{L}\right\rangle^{\mathrm{c}}$ which is finite, whence this contribution vanishes after dividing by $X$. The first term integrates to $\left\langle Q_{I} Q_{J} Q_{K} q_{L}\right\rangle^{\mathrm{c}}$ which is also finite. The second and third terms give contributions that diverge with $X$ (because of space-translation invariance), respectively $2 X\left\langle Q_{I} q_{K}\right\rangle^{\mathrm{c}}\left\langle Q_{J} q_{L}\right\rangle^{\mathrm{c}}$ and $2 X\left\langle Q_{I} q_{L}\right\rangle^{\mathrm{c}}\left\langle Q_{J} q_{K}\right\rangle^{\mathrm{c}}$. Using diagonality $\left\langle Q_{I} q_{K}\right\rangle^{\mathrm{c}}=$ $\delta_{I K}$, we find

$$
\left\langle w_{I J}, w_{K L}\right\rangle_{\mathcal{H}^{\prime \prime}}=\left|v_{K}^{\mathrm{eff}}-v_{L}^{\mathrm{eff}}\right|\left(\delta_{I K} \delta_{J L}+\delta_{I L} \delta_{J K}\right) .
$$

Note that this implies $\left\|w_{I J}\right\|_{\mathcal{H}^{\prime \prime}}=\sqrt{\left|v_{I}^{\text {eff }}-v_{J}^{\text {eff }}\right|}$. In particular, for $I=J$, or whenever $v_{I}^{\text {eff }}=v_{J}^{\text {eff }}$, we find

$$
\left\|w_{I J}\right\|_{\mathscr{H}^{\prime \prime}}=0 \quad\left(v_{I}^{\mathrm{eff}}=v_{J}^{\mathrm{eff}}\right) .
$$

This implies that in these cases, $\mathfrak{w}_{I J}$ are $n u l l$ in $\mathscr{H}^{\prime \prime}$. This is at the root of divergent Onsager matrix elements in Theorem 5.1.

The set of elements $w_{I J} \subset \mathscr{H}^{\prime \prime}$ spans (a dense subset of) what may be called the wave scattering subspace,

$$
\mathcal{H}_{\text {scat }}=\overline{\operatorname{span}\left\{w_{I J}\right\}} \subset \mathscr{H}_{\text {dif }} .
$$

This is indeed a subspace of $\mathcal{H}_{\text {dif }}$, as a consequence of (120). By symmetry, a basis is obtained by considering $I>J$. With this, we may project onto $\mathscr{H}_{\text {scat }}$, and we obtain a lower bound for the matrix $\mathfrak{L}_{a, b}$ defined in (54), and also for the Onsager matrix $\mathfrak{L}_{i j}$ constructed out of the conserved currents. The bound is the matrix

$$
\mathfrak{L}_{a, b}^{\text {scat }}=\sum_{\substack{I>J \\ v_{I}^{\text {eff }} \neq v_{J}^{\text {eff }}}} \frac{\left\langle a, w_{I J}\right\rangle_{\mathrm{dif}}\left\langle w_{I J}, b\right\rangle_{\mathrm{dif}}}{\left|v_{I}^{\mathrm{eff}}-v_{J}^{\mathrm{eff}}\right|},
$$

that is

$$
\mathfrak{L} \geq \mathfrak{L}^{\text {scat }}
$$

In particular,

$$
\mathfrak{L}_{a, a} \geq \sum_{\substack{I>J \\ \text { eff } \\ v_{I} \neq v_{J}^{\text {eff }}}} \frac{\left|\left\langle Q_{I} Q_{J} \cdot\left(1-\mathbb{P}_{\tau}\right) a\right\rangle^{\mathrm{c}}\right|^{2}}{\left|v_{I}^{\mathrm{eff}}-v_{J}^{\mathrm{eff}}\right|}
$$

In fact, Formula (144) suggests the definition of a bilinear map

$$
\begin{aligned}
\phi_{\text {scat }}: \overline{\mathcal{H}_{\text {bal }} \wedge \mathcal{H}_{\text {bal }}} & \rightarrow \mathcal{H}_{\text {dif }} \\
\left(q_{I}, q_{J}\right) & \mapsto \phi_{\text {scat }}\left(q_{I}, q_{J}\right)=w_{I J} .
\end{aligned}
$$

(By (147), the diagonal of $\mathcal{H}_{\text {bal }} \otimes \mathcal{H}_{\text {bal }}$ is indeed omitted in the domain of $\phi_{\text {scat }}$.) This maps onto the subspace $\mathscr{H}_{\text {scat }} \subset \mathscr{H}_{\text {dif }}$,

$$
\operatorname{Ran}\left(\phi_{\text {scat }}\right)=\mathcal{H}_{\text {scat }} .
$$

Recall that $q_{I}$ as an element of $\mathscr{H}$ is defined up to constant shifts $q_{I}(x) \mapsto q_{I}(x)+a \mathbf{1}$ and gauge transformations $q_{I}(x) \mapsto q_{I}(x)+\partial_{x} a(x)$. Thus in order for the map (152) to be well defined, we must verify that the result is invariant under these transformation. This is 
indeed the case: since $q_{I} \equiv 0$ in $\mathcal{H}^{\prime \prime}$, then the constant shift leaves the expression invariant; and the gauge transformation produces, in the integral, boundary terms which are finite in $\mathcal{H}^{\prime \prime}$, hence their contribution vanishes in the limit $X \rightarrow \infty$.

I call $\phi_{\text {scat }}$ the wave scattering map. An interpretation of it is as follows. The elements $\phi_{\text {scat }}\left(q_{I}, q_{J}\right)$ represent hydrodynamic two-body scattering states for ballistic waves, where two interacting, coherent, ballistic waves propagate (the fact that the product of conserved densities are coherently and ballistically transported is expressed mathematically in Formula (189) in Appendix 5). The average over space $x$ in (144) represents the average over all distances between the waves. The factor $\left|v_{I}^{\text {eff }}-v_{J}^{\text {eff }}\right|$ relates to a "density of state": the normalisation factor by which we divide the integral in (144) is not the total distance $2 X$ between the observables, but the total time required for them to reach a distance $2 X$ under ballistic propagation. The range $\mathcal{H}_{\text {scat }}$ of the map is the wave scattering subspace of $\mathcal{H}_{\text {dif }}$. The overlaps $\left\langle\phi_{\text {scat }}\left(q_{I}, q_{J}\right), a\right\rangle_{\mathrm{dif}}=\left\langle w_{I J}, a\right\rangle_{\mathrm{dif}}$ represent the "probability amplitudes" for the wave scattering state to be created by the perturbation $a$. Physically, it can be interpreted in terms of the spreading of the observable $a$ as it evolves: it is the strength of ballistically propagating coherent pulses of modes $I$ and $J$ at the fronts. In general, the spreading is formed of a linear combination of many such fronts. One may interpret the part of the hydrodynamic entropy production (64) due to wave scattering $\mathcal{H}_{\text {scat }}$ (coming from the part of $\|v\|_{\text {dif }}$ obtained by projection onto $\mathcal{H}_{\text {scat }}$ ) as that coming from a redistribution of largescale convective structures towards structures at the lower diffusive scale. Applied to the Onsager matrix, with $a=j_{i}$ and $b=j_{j}$, the lower bound (149) represents the contribution to diffusion from the scattering of ballistic waves. The projection $\left\langle\phi_{\text {scat }}\left(q_{I}, q_{J}\right), j_{i}\right\rangle_{\text {dif }}$ is the amplitude for the current observables to absorb or produce two coherent ballistic waves, and the denominator represents the amplitude of overlap between the waves.

Finally, I observe that for the current observables $j_{i}$, the expression for $\mathfrak{L}_{i j}^{\mathrm{scat}}=\mathfrak{L}_{j_{i}, j_{j}}^{\text {scat }}$ from (149) agrees with the formula obtained in [29] from a proposal akin to nonlinear fluctuating hydrodynamics. It is worth explaining the relation between the formalism of this work and that developed here. In particular, I believe my derivation clarifies three important points, which may be useful for future studies:

- First, the heuristic expansion of the current observable in powers of density observables in [29] has the more precise meaning of an expansion within the Hilbert space $\mathcal{H}_{\text {scat }}$. Hence, the proposal there is valid as an exact formula for observables that lie within $\mathcal{H}_{\text {scat }}$ only. It is important to note that $j_{i}$ generically does not lie within this space, and thus there are corrections (as anticipated in [29]). From the theory developed here, one can further establish that corrections are present for any observable $a \in \mathcal{H}^{\prime \prime}$ that is not invariant under the higher flows, as these do not lie in $\mathcal{H}_{\text {dif }}$.

- Second, the heuristic arguments given in [29] for the ballistic propagation of two normal modes, which is crucial in order to relate space-integrals to time-integrals, is shown here to arise from the linear-response formula proven in Appendix 5. This states how products of conserved densities in three-point functions can be evolved according to their ballistic transport. Crucially, this shows that one needs to project out the conserved space in order for this ballistic transport formula to hold, clarifying why the projection has to be performed in [29].

- Third, the formula (149) for $\mathfrak{L}_{a, b}^{\text {scat }}$ is seen in the present work as arising from a projection on a Hilbert space. This shows that this formula gives in general a lower bound. This is important, for instance, in order to fully justify, as in Theorem 5.2, the argument made in [29] that superdiffusion must occur - diffusion is infinite-if (in my notation) $\left\langle w_{I I}, a\right\rangle_{\mathcal{H}^{\prime \prime}} \neq 0$. 


\subsection{Geometry of the Manifold of States}

Recall Eq. (60) relating $\beta^{i}$-derivatives of averages $\langle a\rangle$ to inner products in $\mathcal{H}_{\text {bal }}$. It has a clear geometrical meaning, obtained by seeing an observable $a$ as a function on the manifold of states. In symbols, if $\omega \in M$ are the maximal entropy states, we may see, for any given $a \in \mathcal{V}$, the average $\langle a\rangle=a(\omega)$ as a function of the state $\omega$. Then, (60) is an equation for the derivative of this function with respect to $\beta^{i}$. Thus we should identify $\mathcal{H}_{\text {bal }}$ with the tangent space at $\omega$, and $q_{i}$ with a basis of tangent vectors. In particular, on the right-hand side of (60), $a \in \mathcal{H}_{\mathrm{bal}}$ is the abstract vector field, which can be expanded in the basis $\left\{q_{i}\right\}$, that represents the derivative of the function $a(\omega)$. The static covariance matrix $C_{i j}$ is the metric of this Riemannian manifold. Thus, according to the results of Sect. 4, the tangent space is the space of linearly extensive conserved charges for $\mathcal{H}$. This is expanded upon in [22], and gives some clarification to the sense in which linearly extensive conserved charges, as used in the literature on GGEs, are indeed those that generate the maximal entropy states.

Two natural questions arise: if variations in $\mathscr{H}_{\mathrm{th}}$ give overlaps in $\mathcal{H}_{\mathrm{bal}}$, then do variations in $\mathcal{H}_{\text {bal }}$ give overlaps in $\mathscr{H}_{\text {dif }}$ ? And, having a Riemannian manifold, could we construct covariant derivatives? These questions are related. The covariant derivatives can be constructed explicitly using the Levi-Civita connection for the metric $C_{i j}$. Since $C_{i j}=-\frac{\partial^{2} f}{\partial \beta^{i} \partial \beta^{j}}$ is the Hessian of the specific free energy $f$, the connection in fact simplifies to $\Gamma_{j k}^{i}=\frac{1}{2} \sum_{l} C^{i l} \frac{\partial}{\partial \beta^{j}} C_{l k}$. The vector elements of the abstract vector field $a$, given by $\left\langle q^{j}, a\right\rangle_{\text {bal }}=\sum_{k} C^{j k}\left\langle q_{k}, a\right\rangle_{\text {bal }}$, are scalars under parallel transport, and thus by definition of the covariant derivative $\nabla_{i}$, we have

$$
\nabla_{i}\left\langle q^{j}, a\right\rangle_{\mathrm{bal}}=\frac{\partial}{\partial \beta^{i}}\left\langle q^{j}, a\right\rangle_{\mathrm{bal}}
$$

By metric covariance, $\nabla_{i}\left\langle q^{j}, a\right\rangle_{\text {bal }}$ is a covariant tensor, hence $\nabla_{i}\left\langle q_{j}, a\right\rangle_{\text {bal }}=\sum_{k} C_{j k} \nabla_{i}$ $\left\langle q^{k}, a\right\rangle_{\text {bal }}$. Thus,

$$
\nabla_{i}\left\langle q_{j}, a\right\rangle_{\text {bal }}=\frac{\partial}{\partial \beta^{i}}\left\langle q_{j}, a\right\rangle_{\text {bal }}-\sum_{k, l}\left[\frac{\partial}{\partial \beta^{i}} C_{j k}\right] C^{k l}\left\langle q_{l}, a\right\rangle_{\text {bal }}=-\left\langle Q_{i} Q_{j}\left(1-\mathbb{P}_{\tau}\right) a\right\rangle^{\mathrm{c}}
$$

where we use the explicit form (50) for the projection, as well as $\left\langle q_{j}, a\right\rangle_{\text {bal }}=\left\langle Q_{j} a\right\rangle^{\mathrm{c}}$ and

$$
-\frac{\partial}{\partial \beta^{i}}\left\langle Q_{j} a\right\rangle^{\mathrm{c}}=\left\langle Q_{i} Q_{j} a\right\rangle^{\mathrm{c}}
$$

We recognise the right-hand side of (155) as (up to a minus sign) the action of the bilinear charge $Q_{i j}^{\prime \prime}$ constructed in Sect. 5.2, see Eq. (119). That is, the covariant derivative is, on the vector field $a$, a quadratically extensive conserved charge for $\mathcal{H}$. Thus, by the results of Sect. 6.1, the space of covariant derivatives (the tangent space to the tangent space) is a subspace of $\mathscr{H}_{\text {dif }}$; it is the wave scattering space $\mathscr{H}_{\text {scat }}$ spanned by the bilinear charges, Eq. (148).

The remark that covariant derivatives must lie in $\mathscr{H}_{\text {dif }}$ also has a geometric underpinning. Since the metric is covariant, we have $\nabla_{i}\left\langle q_{j}, q_{k}\right\rangle_{\text {bal }}=0$. Therefore, seeing the left-hand side of (155) as a linear functional on $a$, it is null on $\mathscr{H}_{\mathrm{bal}}$. This suggests the hydrodynamic reduction $\mathscr{H}^{\prime} \rightarrow \mathscr{H}^{\prime \prime}$, which trivialises $\mathscr{H}_{\text {bal }}$. Further, it is clear from (155) that it is invariant under time evolution $\tau_{t}$. Hence, it acts well on the space of equivalence classes on which $\mathscr{H}^{\prime \prime}$ is built. The result of Sect. 5.2 is of course stronger, showing that, as a linear functional on $a$, the covariant derivative is in fact bounded with respect to $\mathcal{H}^{\prime \prime}$. 
The interpretation as a covariant derivative gives a geometric meaning for the bilinear map (152), with

$$
-\nabla_{i}\left\langle q_{j}, a\right\rangle_{\mathrm{bal}}=\left\langle\phi_{\mathrm{scat}}\left(q_{i}, q_{j}\right), a\right\rangle_{\mathrm{dif}}
$$

\subsection{Integrability}

This section is more speculative and concerns the relation between many-body integrability and the structure of the Hilbert spaces discussed above.

The classical notion of integrability is based on the Liouville condition, that the number of commuting conserver quantities must agree with the number of conservation laws. This leads to the well known solution by quadrature of the system. The extension of this concept to thermodynamic systems, which possess infinitely-many degrees, such as the thermodynamic limit of quantum or classical gases, or field theories, is a challenging problem. Is the presence of infinitely-many conserved quantities sufficient for integrability? The problem is even starker in quantum chains: in finite volumes, there always are as many conserved quantities in involution as the dimension of the quantal Hilbert space: the projectors on the Hamiltonian eigenspaces. How should we define, without recourse to any particular technical solution procedures, the notion of integrability in infinite chains?

A basis for the answer to these questions has been suggested for a long time: one must consider conserved charges with appropriate locality properties. The Hilbert spaces constructed in this paper, and in particular the results [23] on Euler-scale hydrodynamic projections, formalise the notion of locality in a mathematically precise fashion. Thus it is natural to ask if we can define integrability using these. Here I propose that this is the case.

First, one can verify, as is shown in [29], that the expression for $\mathfrak{L}_{i j}^{\text {scat }}$ from (149) reproduces the exact result of $[13,14]$ for the Onsager matrix $\mathfrak{L}_{i j}$ in integrable models. Hence, in integrable models, the wave scattering bound is saturated

$$
\mathfrak{L}_{i j}=\mathfrak{L}_{i j}^{\text {scat }} \quad \text { (integrable systems). }
$$

This observation is in agreement with the interpretation of the calculation in $[13,14]$, which used a spectral decomposition of the current-current two-point function in terms of matrix elements of particle and holes excitations above a finite-density Bethe background. In this calculation, it is indeed seen that only the 2-particle-hole-pair terms in the spectral decomposition contribute to the Onsager matrix, the result being interpreted as a two-body scattering process. The partial expansion formula (149) is a generalisation to arbitrary many-body models of this calculation and of its underlying physical interpretation.

One might want to define integrable systems by the requirement that their diffusion be solely due to wave scattering states. I propose that this observation in fact points to a more fundamental definition of integrability in terms of its space of conserved charges. Recall the setup of Sect. 3.2, in particular the higher flows and their invariants (42). Recall that the wave scattering space - the space of covariant derivatives - is a subspace of the diffusive space,

$$
\mathscr{H}_{\text {scat }} \subset \mathscr{H}_{\text {dif }},
$$

itself invariant under all higher flows (if any), $\mathscr{H}_{\mathrm{dif}}=\mathcal{Q}^{\prime \prime}$. Clearly, if there is only a finite number of conserved flows, then $\mathscr{H}_{\text {dif }}$ is relatively big, while $\mathscr{H}_{\text {scat }}$ is relatively small. The extreme case is if $\iota$ and $\tau$ are the only flows; then $\mathscr{H}_{\text {dif }}=\mathscr{H}^{\prime \prime}$ while $\mathscr{H}_{\text {scat }}$ is composed of a single element $w_{12}$. As the number of conserved quantities increase, the space $\mathcal{H}_{\text {dif }}$ decreases and $\mathscr{H}_{\text {scat }}$ increases: the conserved quantities restrict diffusion, but increase the 
wave scattering contributions within it. Thus, with enough conserved quantities, the spaces may become equal. Therefore I propose the following definition: a many-body system is integrable if and only if there are enough conserved flows in involution such that

$$
\mathcal{H}_{\text {scat }}=\mathscr{H}_{\text {dif }} \quad \text { (definition of integrability). }
$$

That is, in integrable systems, once the complete set of conserved densities $\left\{q_{i}\right\}$ has been found at the ballistic level, giving $\mathscr{H}_{\text {bal }} \subset \mathscr{H}^{\prime}$ and generating conserved flows in involution, then at the diffusive level, in $\mathscr{H}_{\text {dif }} \subset \mathscr{H}^{\prime \prime}$, there are no new conserved degrees of freedom. The geometric meaning is that not only the tangent space of the state manifold generates the space of flow invariants in the first-order hydrodynamic space $\mathscr{H}^{\prime}$, but also the covariant derivatives generate the space of flow-invariants in the second-order hydrodynamic space $\mathcal{H}^{\prime \prime}$.

This definition immediately explains why the wave scattering subspace saturates diffusion, Eq. (158). Indeed, as mentioned current observables are in general invariant under higher flows, hence elements of the diffusive subspace $\mathscr{H}_{\text {dif }}$, Eq. (53). As a consequence, the definition (160) implies that in integrable systems

$$
j_{i} \in \mathcal{H}_{\text {scat }} \text { as an element of } \mathcal{H}_{\text {dif }} \quad \text { (integrable systems) }
$$

and this immediately implies (158).

This proposal appears to be supported by the form factor calculations of [14]. Performing a similar calculation for an arbitrary observable instead of currents, one obtains an infinite series, not only the two particle-hole terms (I refer to [14] for the discussion of particlehole states). However, requiring that the observable be in $\mathcal{H}_{\text {dif }}$, hence invariant under all higher flows (as an element of $\mathscr{H}^{\prime \prime}$, not as a local observable, which would be too strong), all processes in this series must conserved momenta. As only two integrals are made (over space and time), it must be that only the two particle-hole terms remain, and therefore the inner product involving this observable in $\mathscr{H}_{\text {dif }}$ can be projected onto $\mathscr{H}_{\text {scat }}$.

Finally, it is also possible to describe free systems. I note three proposal or sets of results which I now put together. First, it is proposed in [43] that the difference between free and interacting integrable models is that in the former, diffusion vanishes, while in the latter it does not. Second, it is proposed in [44] that free models are exactly those for which the natural flux Jacobian ${ }^{5} \mathrm{~A}_{i}{ }^{j}=\partial\left\langle j_{i}\right\rangle / \partial\left\langle q_{j}\right\rangle$, is independent of the state. Third, it is shown [45] in a large class of spin chain models with "free-particle" description that there is a choice of gauge (a choice of explicit conserved densities and currents in the spin chain) such that the current operators is itself a conserved density. I now show that these statements, along with the proposal (160), agree with the following definition of free models:

$$
j_{i}=0 \text { as an element of } \mathscr{H}_{\text {dif }} \quad \text { (definition of free systems) }
$$

Indeed, if, in an appropriate gauge, current observables are conserved densities, then $j_{i} \in \mathcal{H}_{\text {bal }}$ as elements of $\mathcal{H}^{\prime}$. As a consequence, since $\mathcal{H}_{\text {bal }} \rightarrow\{0\}$ in the hydrodynamic reduction to the diffusive space, (162) holds. Another way of seeing this is that if the natural flux Jacobian is state-independent, then the diagonal basis in Appendix 2 is state-independent, and therefore $\left\langle w_{I J}, j_{j}\right\rangle_{\mathcal{H}^{\prime \prime}}=0$, from Eq. (119). Hence, $\mathbb{P}_{\text {scat }} j_{i}=0$, and combined with my proposal for integrability (160) that $j_{i} \in \mathcal{H}_{\text {scat }}$, this gives (162). Clearly, with (162), the Onsager matrix vanishes, in agreement with the proposal [43].

\footnotetext{
5 The natural flux Jacobian is that obtained in any system of coordinates, on the manifold of maximal entropy states, that is linear in the average conserved densities $\left\langle q_{i}\right\rangle$. For instance, in general, the system formed by the normal modes at every point on the manifold is nonlinear. See [44].
} 


\section{Conclusion}

I have described a procedure for constructing certain Hilbert spaces which give information about the hydrodynamic properties of a many-body system. This is a general procedure, which generalises the standard setup of ballistic hydrodynamic projections. In particular, I have defined the second order hydrodynamic space $\mathscr{H}^{\prime \prime}$, in which the diffusive Hilbert space lies $\mathcal{H}_{\text {dif }} \subset \mathcal{H}^{\prime \prime}$. The diffusive space is the space of invariants under higher Hamiltonian flows, if there are any such flows (such as in integrable systems); otherwise it is all of $\mathcal{H}^{\prime \prime}$. I have explained how the Onsager matrix, describing diffusion, is written as an inner product in $\mathscr{H}_{\text {dif }}$. I have also defined fractional-order spaces $\mathscr{H}^{(u)}$, and those lying between the ballistic and diffusive spaces, $u \in(1,2)$, are associated with superdiffusion.

I have shown that quadratically extensive conserved charges give elements of $\mathcal{H}^{\prime \prime}$. The construction gives a slightly different, and stronger, version of the lower bound for diffusion found by Prosen [10]. I have explained how one can write a hydrodynamic projection formula for the Onsager matrix which extends such lower bounds to a formally exact expression, much like standard hydrodynamic projections for the Drude weights [1,21] extend the Mazur bound [24-28] based on linearly extensive charges.

I have constructed particular elements of $\mathcal{H}_{\mathrm{dif}}$ associated to bilinear expressions in linearly extensive conserved charges. Their contribution to diffusion describes the effects of the scattering of ballistic waves; their overlaps with local observables can be interpreted as representing the ballistic fronts of observable spreading. These elements span what I call the wave scattering space $\mathscr{H}_{\text {scat }} \subset \mathscr{H}_{\text {dif }}$. Geometrically, they describe the covariant derivatives on the manifold of maximal entropy states.

The action of bilinear charges are completely calculable by the knowledge of the averages of local observables. They give rise to explicit lower bounds for the Onsager matrix, whose strength depends on the clustering properties of three-point functions. Using solely clustering of the Lieb-Robinson type, I show that nonzero diffusion can be established from the evaluation of an integrated three-point function, available from the thermodynamic averages of currents. Using an Euler-scale linear response principle, I have obtained expressions for three-point functions involving conserved densities, which suggest sharper clustering properties based on hydrodynamic propagation velocities. These sharper clustering properties imply a stronger diffusion lower bounds. Further, from the three-point function result, the full projection onto $\mathscr{H}_{\text {scat }}$ is evaluated, giving a matrix lower bound. This matrix lower bound agrees with that conjectured recently using different, less rigorous methods [29], and is saturated in integrable models [13-15].

Assuming that clustering is controlled by hydrodynamic velocities, I have shown that if the diagonal $G$-coupling of nonlinear fluctuating hydrodynamics (NFH) is nonzero, then there must be superdiffusion (the associated Onsager matrix element is infinity). Assuming clustering properties inferred from the general superdiffusion phenomenology further implies that the superdiffusive exponent is bounded from below by $2 / 3$ if the scaling function has finite variance, the exponent of the KPZ universality class. I likewise bound the heat-mode exponent by $3 / 5$ and recover the Fibonacci sequence predicted in the context of NFH. These are obtained without the use of the hydrodynamic equation, and without adding noise or using mode-coupling theory, and give a strong confirmations of results from NFH.

I have speculated that an accurate definition of integrability can be obtained by the coincidence of $\mathcal{H}_{\text {scat }}$ with $\mathcal{H}_{\text {dif }}$ : with enough conserved quantities in involution, diffusion is limited enough, yet there is enough wave scattering, so that diffusion is purely from scattering of ballistic waves. This parallels the usual statement that integrability is the presence of as many 
conserved quantities in involution as degrees of freedom. I noted that generically, observables, in integrable and non-integrable systems, are not supported on the wave scattering subspace, this being a particularity of current observables in integrable systems. Generic observables therefore have more involved spreading structure. In free models, current observables are null in the diffusive space, hence there is no diffusion.

Adding the complementary space $\mathscr{H}_{1}$ within the diffusive space, $\mathscr{H}_{\text {dif }}=\mathscr{H}_{\text {scat }} \oplus \mathcal{H}_{1}$, and $\mathscr{H}_{2}$ within the second order hydrodynamic space, $\mathscr{H}^{\prime \prime}=\mathscr{H}_{\text {dif }} \oplus \mathscr{H}_{2}$, we have

$$
\mathcal{H}^{\prime \prime}=\mathscr{H}_{\text {scat }} \oplus \mathcal{H}_{1} \oplus \mathcal{H}_{2} \text {. }
$$

The spaces $\mathscr{H}_{1}$ and $\mathscr{H}_{2}$ will be studied in forthcoming works. In particular, $\mathscr{H}_{2}$ is identified with the physics of thermalisation [38]. I also point out that one might get insight by considering the various hydrodynamic structures that may form besides ballistically propagating waves. For instance, in general, shocks may develop, which will contribute to diffusion. This points to the idea of constructing asymptotic hydrodynamic states based on such structures, giving rise to hydrodynamic spectral decompositions of multi-point functions paralleling the form factor expansion in integrable models [14,46,47].

A particular family of fluid equations, of which the hydrodynamics of integrable systems is part, is that of linearly degenerate fluids, see [48-51]. Linearly degenerate fluids do not develop shocks, and one might therefore conjecture that they admit purely wave scattering diffusion. It would be interesting to study wave scattering processes in linearly degenerate hydrodynamics, and understand how they connect with integrable systems in more depth.

The sequential construction (44) points to the possibility of studying finer hydrodynamic scales when higher flows are available (such as in integrable systems). Effectively, in the hydrodynamic equation probing the finer scales, at the $n^{\text {th }}$ stage of the sequence, one expands the dispersion relation of the $n^{\text {th }}$ flow to the $n^{\text {th }}$ power of momentum, as the lower powers are projected out by the lower stages. There is no need to choose the sequence of flows in any particular order (for instance, any flow of the hierarchy can be used for generating time evolution), but in certain situations, it may be natural to order the flows by the power of momentum they correspond to (for instance, in Galilean systems the momentum is $p$, the energy $p^{2} / 2$, and other linearly extensive conserved charges of integrable systems carry higher powers of $p$ ). Naturally, extending (87), these higher spaces should be spanned by charges of higher-order extensivity. Importantly, the construction suggests that there are as many meaningful hydrodynamic scales as there are commuting flows; in particular, in conventional, non-integrable systems $(N=2)$, this suggests that there are no hydrodynamic scales beyond the diffusive scale: the hydrodynamic assumption that conserved densities on time slices fully describe the local averages is broken. In integrable systems $(N=\infty)$, there are infinitely many hydrodynamic scales, supposedly forming an asymptotic expansion of local averages.

Fractional-order spaces $\mathcal{H}^{(u)}$ may also be nontrivial for orders $u \in(0,1)$, that is, lying between the thermodynamic and ballistic spaces. In one dimension, thermal states for local quantum-chain hamiltonians are exponentially clustering, hence such fractional spaces are trivial. However, at zero temperature, there may be quantum phase transitions, where clustering is power-law and spaces of fractional orders between 0 and 1 should become nontrivial. Hence, at orders $u \in(0,1)$, nontrivial fractional order spaces are associated with quantum criticality, and at orders $u \in(1,2)$, they are associated with superdiffusion, which can be argued to correspond to dynamical phase transitions [44].

Finally, it is clear that, as for the linearly extensive charges [22], most aspects of the present theory can be generalised to higher-dimensional models, where it would be interesting to connect with proposed general results, e.g. [52]. 
Acknowledgements This work benefited from discussions with O. A. Castro-Alvaredo, J. De Nardis, W. De Roeck, M. Medenjak, T. Prosen, T. Sasamoto, H. Spohn and T. Yoshimura. I am particularly grateful to T. Yoshimura for showing me the main result of [29] before publication while in Tokyo. I acknowledge funding from the Royal Society under a Leverhulme Trust Senior Research Fellowship, "Emergent hydrodynamics in integrable systems: non-equilibrium theory", ref. SRF\R1\180103, and for the last additions to the paper (in particular Sect. 2), from the EPSRC under the grant "Emergence of hydrodynamics in many-body systems: new rigorous avenues from functional analysis", ref. EP/W000458/1. I am grateful to the Tokyo Institute of Technology for support and hospitality during an invited professorship (October 2019), where this research was started. This research was also supported in part by the International Centre for Theoretical Sciences (ICTS) during a visit for the program Thermalization, Many body localization and Hydrodynamics (code: ICTS/hydrodynamics2019/11).

Open Access This article is licensed under a Creative Commons Attribution 4.0 International License, which permits use, sharing, adaptation, distribution and reproduction in any medium or format, as long as you give appropriate credit to the original author(s) and the source, provide a link to the Creative Commons licence, and indicate if changes were made. The images or other third party material in this article are included in the article's Creative Commons licence, unless indicated otherwise in a credit line to the material. If material is not included in the article's Creative Commons licence and your intended use is not permitted by statutory regulation or exceeds the permitted use, you will need to obtain permission directly from the copyright holder. To view a copy of this licence, visit http://creativecommons.org/licenses/by/4.0/.

\section{Appendix 1: Current Correlation Asymptotics in Non-correlated Particle Systems}

Without loss of generality we assume $\mathbf{v}=0$ in (2). We derive Eq. (8) as follows:

$$
\begin{aligned}
\int_{0}^{t} \mathrm{~d} s \int \mathrm{d}^{d} x\left\langle j^{i}(\mathbf{x}, s) j^{i}(0,0)\right\rangle^{\mathrm{c}} & =\frac{1}{2} \int_{0}^{t} \mathrm{~d} s \int \mathrm{d}^{d} x \partial_{i} \partial_{k}|\mathbf{x}|^{2}\left\langle j^{i}(\mathbf{x}, s) j^{k}(0,0)\right\rangle^{\mathrm{c}} \\
& =\frac{1}{2} \int_{0}^{t} \mathrm{~d} s \int \mathrm{d}^{d} x|\mathbf{x}|^{2} \partial_{i} \partial_{k}\left\langle j^{i}(\mathbf{x}, s) j^{k}(0,0)\right\rangle^{\mathrm{c}} \\
& =-\frac{1}{2} \int_{0}^{t} \mathrm{~d} s \int \mathrm{d}^{d} x|\mathbf{x}|^{2}\left\langle\partial_{i} j^{i}(\mathbf{x}, s) \partial_{k} j^{k}(0,0)\right\rangle^{\mathrm{c}} \\
& =-\frac{1}{2} \int_{0}^{t} \mathrm{~d} s \int \mathrm{d}^{d} x|\mathbf{x}|^{2}\left\langle\left.\partial_{s} n(\mathbf{x}, s) \partial_{s^{\prime}} n\left(0, s^{\prime}\right)\right|_{s^{\prime}=0}\right\rangle^{\mathrm{c}} \\
& =\frac{1}{2} \int_{0}^{t} \mathrm{~d} s \int \mathrm{d}^{d} x|\mathbf{x}|^{2} \partial_{s}^{2}\langle n(\mathbf{x}, s) n(0,0)\rangle^{\mathrm{c}} \\
& =\left.\frac{1}{2} \partial_{s} \int \mathrm{d}^{d} x|\mathbf{x}|^{2}\langle n(\mathbf{x}, s) n(0,0)\rangle^{\mathrm{c}}\right|_{s=0} ^{t} \\
& \sim \frac{\chi}{2} \partial_{t}\left[g(t)^{2} \Delta f\right] \\
& =\chi \Delta f g^{\prime}(t) g(t)
\end{aligned}
$$

where

$$
\Delta f=\int \mathrm{d}^{d} x|\mathbf{x}|^{2} f(\mathbf{x})
$$

is assumed finite.

In the fat-superdiffusion case with $d=1$, where $\Delta f=\infty$, we use $\langle n(x, t) n(0,0)\rangle^{\mathrm{c}} \sim$ $c t|x|^{-1-z}$ and integrate over a spatial region growing proportionality to $t$ (see the discussion 
around Eq. (11)), to get

$$
\begin{aligned}
\int_{0}^{t} \mathrm{~d} s \int_{-a t}^{b t} \mathrm{~d} x\langle j(x, s) j(0,0)\rangle^{\mathrm{c}} & =\left.\frac{1}{2} \int_{-a t}^{b t} \mathrm{~d} x|x|^{2} \partial_{s}\langle n(x, s) n(0,0)\rangle^{\mathrm{c}}\right|_{s=0} ^{t} \\
& \sim \frac{c\left(a^{2-z}+b^{2-z}\right)}{2-z} t^{2-z} .
\end{aligned}
$$

The velocities $a, b>0$ are here phenomenological; in a more accurate treatment, they are obtained from the bounding modes surrounding the fat-superdiffusive mode. The coefficient $c$ is not simply related to the susceptibility; it simply controls the asymptotics of the density two-point function.

\section{Appendix 2: Diagonal Basis of Conserved Quantities}

In a thermodynamic state, assumed invariant under all flows generated by the $Q_{i}$ 's, one defines the following hydrodynamic matrices:

$$
\begin{aligned}
\mathrm{C}_{i j} & =\left\langle q_{i}, q_{j}\right\rangle_{\mathcal{H}^{\prime}}=\left\langle Q_{i} q_{j}\right\rangle^{\mathrm{c}} \\
\mathrm{B}_{i j} & =\left\langle q_{i}, j_{j}\right\rangle_{\mathcal{H}^{\prime}}=\left\langle Q_{i} j_{j}\right\rangle^{\mathrm{c}} \\
\mathrm{A}_{i}{ }^{j} & =\frac{\partial\left\langle j_{i}\right\rangle}{\partial\left\langle q_{j}\right\rangle}=\sum_{k} \mathrm{~B}_{i k} \mathrm{C}^{k j}
\end{aligned}
$$

where $C^{i j}$ is the inverse of the matrix $C_{i j}$. By general principles $[2,12,14,53], B_{i j}=B_{j i}$, implying

$$
A C=C A^{T}
$$

The matrix $C_{i j}$ is like a "metric", used to raise and lower indices.

It is known that one can diagonalise the flux Jacobian A,

$$
\mathrm{A}=\mathrm{R}^{-1} v^{\mathrm{eff}} \mathrm{R}
$$

where $v^{\text {eff }}=\operatorname{diag}\left(v_{I}^{\text {eff }}\right)$ is the diagonal matrix of normal-mode velocities of the Euler hydrodynamics of the model. The columns of the matrix $R^{-1}$ are the eigenvectors of $A$. These eigenvectors can be chosen to form an orthonormal basis with respect to the form given by the matrix $C^{-1}$, and therefore we can choose $R$ to satisfy

$$
\mathrm{RCR}^{\mathrm{T}}=\mathbf{1} \quad\left(\sum_{j k} \mathrm{R}_{i}{ }^{j} \mathrm{C}_{j k} \mathrm{R}_{l}{ }^{k}=\delta_{i l}\right) .
$$

It is convenient to introduce the normal-mode basis, labelled by capital-letter indices $I, J, K, \ldots$, by transforming vectors as

$$
q_{I}=\sum_{i} \mathrm{R}_{I}^{i} q_{i}
$$

In this basis,

$$
C_{I J}=\left\langle q_{I}, q_{J}\right\rangle_{\mathcal{H}^{\prime}}=\sum_{i j} \mathrm{R}_{I}^{i} \mathrm{C}_{i j} \mathrm{R}_{J}^{j}=\left[\mathrm{RCR}^{\mathrm{T}}\right]_{I J}=\delta_{I J}
$$


Hence in this basis, the metric is the identity. In particular, we have

$$
\mathbb{P}_{\tau} a=\sum_{I} q_{I}\left\langle q_{I}, a\right\rangle_{\mathcal{H}^{\prime}}
$$

and

$$
\mathrm{D}_{a, b}=\sum_{I}\left\langle a, q_{I}\right\rangle_{\mathscr{H}^{\prime}}\left\langle q_{I}, b\right\rangle_{\mathcal{H}^{\prime}}=\sum_{I}\left\langle a \cdot Q_{I}\right\rangle^{\mathrm{c}}\left\langle Q_{I} \cdot b\right\rangle^{\mathrm{c}} .
$$

The diagonal basis is state-dependent, except in free systems.

\section{Appendix 3: Currents as Invariants}

This is based on Sect. 3.3. It is sufficient to consider the infinitesimal generators of the flows, $\delta_{\ell}=\lim _{s \rightarrow 0}\left(\tau_{s}^{(\ell)}-1\right) / s$. By (42), all conserved densities $q_{i}$ 's are invariant under all flows in $\mathcal{H}^{\prime}$. Then, in $\mathcal{H}^{\prime}$, we have $\mathbb{P}_{\tau}\left(\delta_{\ell} a\right)=0$ because $\left\langle\delta_{\ell} a, q_{i}\right\rangle_{\mathcal{H}^{\prime}}=-\left\langle a, \delta_{\ell} q_{i}\right\rangle_{\mathcal{H}^{\prime}}=0$. Therefore, in terms of local observables instead of elements of $\mathcal{H}^{\prime}$, every flow generates its currents, so that $\delta_{\ell} q_{i}+\partial_{x} j_{i}^{(\ell)}=0$. Hence, we have (here $j_{i}=j_{i}^{(2)}$ is the "usual" current)

$$
\begin{aligned}
\left\langle\delta_{\ell} j_{i}, a\right\rangle_{\mathcal{H}^{\prime \prime}} & =\lim _{T \rightarrow \infty} \int_{-T}^{T} \mathrm{~d} t \int \mathrm{d} x\left\langle\delta_{\ell} j_{i}(x, t) \cdot a(0,0)\right\rangle^{\mathrm{c}} \\
& =-\lim _{T \rightarrow \infty} \int_{-T}^{T} \mathrm{~d} t \int \mathrm{d} x x\left\langle\delta_{\ell}\left(\partial_{x} j_{i}(x, t)\right) \cdot a(0,0)\right\rangle^{\mathrm{c}} \\
& =\lim _{T \rightarrow \infty} \int_{-T}^{T} \mathrm{~d} t \int \mathrm{d} x x\left\langle\delta_{\ell}\left(\partial_{t} q_{i}(x, t)\right) \cdot a(0,0)\right\rangle^{\mathrm{c}} \\
& =\lim _{T \rightarrow \infty} \int \mathrm{d} x x\left\langle\delta_{\ell}\left(q_{i}(x, T)-q_{i}(x,-T)\right) \cdot a(0,0)\right\rangle^{\mathrm{c}}
\end{aligned}
$$

and using the current for the $\ell$ th flow,

$$
\begin{aligned}
& =-\lim _{T \rightarrow \infty} \int \mathrm{d} x x \partial_{x}\left\langle\left(j_{i}^{(\ell)}(x, T)-j_{i}^{(\ell)}(x,-T)\right) \cdot a(0,0)\right\rangle^{\mathrm{c}} \\
& =\lim _{T \rightarrow \infty} \int \mathrm{d} x\left\langle\left(j_{i}^{(\ell)}(x, T)-j_{i}^{(\ell)}(x,-T)\right) \cdot a(0,0)\right\rangle^{\mathrm{c}} \\
& =\lim _{T \rightarrow \infty}\left\langle j_{i}^{(\ell)}(T)-j_{i}^{(\ell)}(-T), a\right\rangle_{\mathcal{H}^{\prime}} \\
& =\left\langle\mathbb{P}_{\tau}\left(j_{i}^{(\ell)}-j_{i}^{(\ell)}\right), a\right\rangle_{\mathcal{H}^{\prime}} \\
& =0 .
\end{aligned}
$$

In the penultimate step, I used a weak assumption of projection at infinity for $\mathscr{H}^{\prime}$, simply that the large time limit is the projection. Therefore, $\left[Q_{\ell}, j_{i}\right]=0$ in $\mathcal{H}^{\prime \prime}$, and thus $j_{i} \in \mathcal{Q}^{\prime \prime}=\mathscr{H}_{\mathrm{dif}}$.

\section{Appendix 4: Superdiffusive Scaling of Current Correlation Functions}

For the general argument leading to these precise conditions, consider the $V$-setup of local observables and their expectation values, and recall the phenomenology of superdiffusivity. This purports that the two-point functions of a superdiffusive normal mode $q_{I}$ (see Appendix

2) take a scaling form at large $x, t$ 


$$
\left\langle q_{I}(x, t) q_{I}(0,0)\right\rangle^{\mathrm{c}} \sim \frac{1}{|\lambda t|^{\alpha}} f\left(\frac{x-v_{I}^{\mathrm{eff}} t}{|\lambda t|^{\alpha}}\right)
$$

where $f(z)$ integrates to $1, \lambda>0$ and $\alpha \in(1 / 2,1)$ (the case $\alpha=1 / 2$ would be normal diffusion). For the purpose of this discussion, it is sufficient to assume that $v_{I}^{\text {eff }}=0$ and that $f(z)$ be symmetric. This does not affect the scaling analysis; for instance, $v_{I}^{\text {eff }}=0$, for a given $I$, in many cases can be achieved by appropriate Gallilean or relativistic boosts. In (177) and below, we must take $q_{I} \in \mathcal{V}$, and use (50) on $\mathcal{V}$. See Remark 3.5 and Sect. 5.1.

The precise shape of $f(z)$, which can be obtained from NFH, is not important for the present consideration. It will be crucial, however, to distinguish two classes: "normal superdiffusion", where $f(z)$ decays fast enough at large $|z|$ in order to have finite variance

$$
\int \mathrm{d} z z^{2} f(z)<\infty \quad \text { (normal superdiffusion), }
$$

and "fat superdiffusion", where $f(z)$ has infinite variance. In the latter case, a more accurate characterisation is necessary. Results from NFH [2] suggest that the normalisation coefficient of the fat tail grows proportionally to $t,\left\langle q_{I}(x, t) q_{I}(0,0)\right\rangle^{\mathrm{c}} \propto|t||x|^{\nu}$ for some $v$, for $|x| \gg$ $|\lambda t|^{\alpha}$ and at large $|t|$. A possible phenomenological explanation is that at very large distances, the fat tail of superdiffusion should be controlled by ballistic processes, and that with these, the correlation grows, at fixed position, linearly in time. Thus, in the fat superdiffusion case, one may expect

$$
f(z) \sim c|z|^{-\left(1+\frac{1}{\alpha}\right)} \quad \text { (fat superdiffusion) }
$$

for some $c>0$, at large $|z|$.

By applying time derivatives and space integrals, one extracts the appropriately decaying current-current correlation function. Then (177) implies that

$$
\left\langle\left(1-\mathbb{P}_{\tau}\right) j_{I}(x, t)\left(1-\mathbb{P}_{\tau}\right) j_{I}(0,0)\right\rangle^{\mathrm{c}} \sim \frac{\alpha \lambda^{2}}{|\lambda t|^{2-\alpha}}\left[(1-2 \alpha) h(z)+\alpha \partial_{z}(z h(z))\right], \quad z=\frac{x}{|\lambda t|^{\alpha}}
$$

where $h(z)=\int_{-\infty}^{z} \mathrm{~d} y y f(y)$. This is shown as follows. For normal modes, as the flux Jacobian has been diagonalised, $\left(1-\mathbb{P}_{\tau}\right) j_{I}=j_{I}-v_{I}^{\text {eff }} q_{I}$, and as mentioned we assume $v_{I}^{\text {eff }}=0$, which simplifies the calculation. Taking time derivatives,

$$
\partial_{t}^{2}\left\langle q_{I}(x, t) q_{I}(0,0)\right\rangle^{\mathrm{c}} \sim \frac{\alpha}{(\lambda t)^{\alpha} t^{2}}\left[\alpha \partial_{z}^{2}\left(z^{2} f(z)\right)+(1-\alpha) \partial_{z}(z f(z))\right]
$$

where $z=x /(\lambda t)^{\alpha}$. By symmetry,

$$
\int \mathrm{d} z, z(z)=0
$$

hence we can set $z f(z)=\partial_{z} h(z)$ with $h(z) \rightarrow 0$ as $|z| \rightarrow \infty$. We find

$$
\begin{aligned}
\partial_{t}^{2}\left\langle q_{I}(x, t) q_{I}(0,0)\right\rangle^{\mathrm{c}} & \sim \partial_{x}^{2}\left(\frac{\alpha(\lambda t)^{\alpha}}{t^{2}}\left[\alpha z^{2} f(z)+(1-\alpha) h(z)\right]\right) \\
& =\partial_{x}^{2}\left(\frac{\alpha(\lambda t)^{\alpha}}{t^{2}}\left[\alpha \partial_{z}(z h(z))+(1-2 \alpha) h(z)\right]\right)
\end{aligned}
$$

from which we deduce (180) (using space-time translation invariance and the conservation law (48) for $q_{I}$ and $j_{I}$ ). 
We must now distinguish the two cases. In the normal superdiffusion case, integrating over $x$, we have

$$
\left\langle\left(1-\mathbb{P}_{\tau}\right) \tau_{t} j_{I}, j_{I}\right\rangle_{\mathcal{H}^{\prime}}=|t|^{2 \alpha-2} \lambda^{2 \alpha} \alpha(2 \alpha-1) \int \mathrm{d} z z^{2} f(z)(1+o(t))
$$

at large $|t|$. As the variance is finite, this is the correct asymptotics.

In contrast, in the fat superdiffusion case, notwithstanding the overall normalisation coefficient, the space integral of the right-hand side of (180) does not exist, as it decays as $|x|^{1-1 / \alpha}$. However, the space integral of the current-current connected correlation function still exists, as this is a two-point function of local observables. The resolution is that the asymptotic power law in (180) is valid, for any given $t$, only in a (large) region of space around $x=0$. This region grows at most proportionally to $t$ if there is a Lieb-Robinson bound. In fact, one would expect the region to be determined by the available hydrodynamic velocities. For instance, for the heat mode in anharmonic chains, which is found to have fat superdiffusion by NFH, the region lies between the two sound modes, see the discussion in [2]. Therefore, integrating over this region, one obtains

$$
\left\langle\left(1-\mathbb{P}_{\tau}\right) \tau_{t} j_{I}, j_{I}\right\rangle_{\mathcal{H}^{\prime}} \propto|t|^{1-\frac{1}{\alpha}}(1+o(t))
$$

where the nonzero proportionality constant depends on the growth rate of the region, and is not important in what follows. This discussion suggests that fat superdiffusion can only occur for modes whose ballistic trajectories lie between that of other modes; these provide the linearly increasing region necessary to contain the power-law decay (however I will not investigate this aspect further).

Note that using (179) in the exact asymptotic (180), one in fact finds a vanishing leadingasymptotic coefficient, $(1-2 \alpha) h(z)+\alpha \partial_{z}(z h(z)) \sim 0 \times z^{1-1 / \alpha}$. However, the precise coefficient of the asymptotic as evaluated from (179) is not expected to be meaningful, as it is affected by other modes at large distances $|x| \gg|\lambda t|^{\alpha}$. Thus only the power law of the asymptotic should be meaningful, and (185) is expected to hold.

The discussion from Eqs. (177) to (185) is based on the phenomenology of superdiffusion. The form (177) says that the normal modes propagate ballistically, with a superdiffusive extension controlled by the exponent $\alpha$ around the ballistic trajectory of velocity $v_{I}^{\text {eff }}$. Both results (184) and (185) lead to the divergence of the Onsager matrix element $\mathcal{L}_{I I}$, because the resulting power law is not integrable. Thus, $j_{I}$ is not an element of $\mathscr{H}^{\prime \prime}$, but instead

$$
\begin{array}{ll}
j_{I} \in \mathcal{H}^{(3-2 \alpha)} & \text { (normal superdiffusion) } \\
j_{I} \in \mathcal{H}^{(1 / \alpha)} & \text { (fat superdiffusion) }
\end{array}
$$

according to (72). This implies in particular the slightly weaker statements (78) and (79).

Note that (79) was phenomenologically justified via the power-law requirement (179). Different power laws would lead to different definitions of fat superdiffusion, and change some of the results below. The precise power law (179), giving (79), therefore appears to be a stronger assumption than the simpler finite-variance assumption of normal superdiffusion, giving (78). However, the fact that in both cases, the full range $u \in(1,2)$ is covered by the superdiffusion range $\alpha \in(1 / 2,1)$, is an indication that these may indeed be generic. 


\section{Appendix 5: Hydrodynamic Projections for Three-Point Functions}

Euler-scale correlation functions $\left\langle a_{1}\left(x_{1}, t_{1}\right) a_{2}\left(x_{2}, t_{2}\right) \cdots a_{x}\left(x_{n}, t_{n}\right)\right\rangle^{\text {eul }}$ are connected correlation functions of local observables at large space-time scales,

$$
\lim _{\lambda \rightarrow \infty} \lambda^{-n+1}\left\langle\overline{a_{1}}\left(\lambda x_{1}, \lambda t_{1}\right) \overline{a_{2}}\left(\lambda x_{2}, \lambda t_{2}\right) \cdots \overline{a_{n}}\left(\lambda x_{n}, \lambda t_{n}\right)\right\rangle^{\mathrm{c}},
$$

where $\overline{a_{i}}\left(\lambda x_{i}, \lambda t_{i}\right)$ are averages over "fluid cells" of extent $o(\lambda)$ around the space-time points $\left(\lambda x_{i}, \lambda t_{i}\right)$. Fluid cell averaging may be done in a variety of ways, see for instance the discussion in [1] and in [42,54]. Euler-scale correlation functions may be evaluated by the linear-response principles proposed in [42].

Here, I show two fundamental formulae for Euler-scale three-point correlation functions using linear response arguments. The arguments are formal, and I will consider the classical case for simplicity. I expect the results, being at the Euler scale, to apply in the quantum case as well.

The first result is

$$
\left\langle\left(1-\mathbb{P}_{\tau}\right) a(x, t) \mathcal{b}(y, 0) c(z, 0)\right\rangle^{\mathrm{eul}}=\left\langle\left(1-\mathbb{P}_{\tau}\right) a(x, t) \mathbb{P} \mathcal{b}(y, 0) \mathbb{P} c(z, 0)\right\rangle^{\mathrm{eul}}
$$

where here and below $\mathbb{P}=\mathbb{P}_{\tau}$. Thus, upon projecting out conserved densities at $(x, t)$, the only contribution from observables at time 0 come from their projection on the conserved densities. Recall that the projection is with respect to the inner product $\langle a, b\rangle:=\langle a, b\rangle_{\mathcal{H}^{\prime}}=$ $\int \mathrm{d} x\langle a(x) b(0)\rangle^{\mathrm{c}}$, and is written explicitly as (50):

$$
\mathbb{P}_{\tau} a=\sum_{i j} q_{i} C^{i j}\left\langle q_{j}, a\right\rangle .
$$

The second formula is

$$
\left\langle\left(1-\mathbb{P}_{\tau}\right) a(x, t) q_{k}(y, 0) q_{l}(z, 0)\right\rangle^{\mathrm{eul}}=\left\langle\left(1-\mathbb{P}_{\tau}\right) a, q_{i}, q_{j}\right\rangle \mathrm{S}_{k}^{i}(x-y, t) \mathrm{S}_{l}^{j}(x-z, t)
$$

where the three-point coupling is

$$
\left\langle\mathfrak{b}, q_{i}, q_{j}\right\rangle=\int \mathrm{d} y \mathrm{~d} z\left\langle\mathcal{b}(0) q_{i}(y) q_{j}(z)\right\rangle^{\mathrm{c}} .
$$

That is, upon projecting out conserved densities at $x, t$, conserved densities at $y, 0$ and $z, 0$ can brought to time $t$ by their Euler-scale propagators, and the overlap with the observable at $x, t$ is the three-point coupling. Recall that, at the Euler scale

$$
\mathrm{S}_{j}^{i}(x, t)=\left[\mathrm{C}^{-1} \delta(x-\mathrm{A} t) \mathrm{C}\right]_{j}^{i} .
$$

Proof A proof for (187) and (189) is as follows. Consider the average $\langle a(x, t)\rangle$ as evolved from an inhomogeneous initial state. By linear response [42], one can insert the Euler-cell averages of $b(y, 0)$ and $c(z, 0)$ by deformation of their conjugate thermodynamic potential in the initial condition, $\beta^{a}(y)$ and $\beta^{b}(z)$, respectively. We evaluate the final result in the homogeneous, stationary state (the latter is not necessary for the general linear response argument, but sufficient for our purposes). This gives:

$$
\begin{aligned}
\langle a(x, t) \mathcal{b}(y, 0) c(z, 0)\rangle^{\mathrm{eul}} & =\frac{\delta}{\delta \beta^{c}(z)} \frac{\delta}{\delta \beta^{b}(y)}\langle a(x, t)\rangle \\
& =\frac{\delta}{\delta \beta^{c}(z)}\left[\left\langle a, q_{i}\right\rangle_{x, t} C_{x, t}^{i j}\left\langle q_{j}(x, t) \mathcal{b}(y, 0)\right\rangle^{\mathrm{eul}}\right] .
\end{aligned}
$$


We evaluate the derivative by applying it to each factor, and then specialising to the stationary and homogeneous state. Applying it to the last factor $\left\langle q_{j}(x, t) \mathcal{b}(y, 0)\right\rangle^{\text {eul }}$, this gives the term

$$
\left\langle a, q_{i}\right\rangle C^{i j}\left\langle q_{j}(x, t) b(y, 0) c(z, 0)\right\rangle^{\mathrm{eul}}
$$

as, by definition, the derivative inserts the observable $c(z, 0)$. The derivative of the first factor $\left\langle a, q_{i}\right\rangle_{x, t}=\int \mathrm{d} y\left\langle a(x, t) q_{i}(y, t)\right\rangle^{\mathrm{c}}$, is $\int \mathrm{d} y\left\langle a(x, t) c(z, 0) q_{i}(y, t)\right\rangle^{\mathrm{c}}=\left\langle a(x, t) c(z, 0), q_{i}\right\rangle$, so we obtain the term

$$
\left\langle a(x, t) c(z, 0), q_{i}\right\rangle C^{i j}\left\langle q_{j}(x, t) \mathcal{b}(y, 0)\right\rangle^{\mathrm{eul}} .
$$

On the term $C_{x, t}^{i j}$, the inverse of the matrix $C$, we use the rule of differentiation of inverse matrices and obtain similarly

$$
-\left\langle a, q_{i}\right\rangle C^{i j}\left\langle q_{j}(x, t) c(z, 0), q_{k}\right\rangle C^{k l}\left\langle q_{l}(x, t) b(y, 0)\right\rangle^{\mathrm{eul}} .
$$

Using (188), the result is

$$
\left\langle\left(1-\mathbb{P}_{\tau}\right) a(x, t) b(y, 0) c(z, 0)\right\rangle^{\mathrm{eul}}=\left\langle\left(1-\mathbb{P}_{\tau}\right) a(x, t) c(z, 0), q_{i}\right\rangle C^{i j}\left\langle q_{j}(x, t) b(y, 0)\right\rangle^{\mathrm{eul}} .
$$

Specialising to $\mathcal{b}(y, 0)=q_{k}(y, 0)$, this gives

$$
\left\langle\left(1-\mathbb{P}_{\tau}\right) a(x, t) c(z, 0) q_{k}(y, 0)\right\rangle^{\mathrm{eul}}=\left\langle\left(1-\mathbb{P}_{\tau}\right) a(x, t) c(z, 0), q_{i}\right\rangle S_{k}^{i}(x-y, t) .
$$

Specialising to $c(z, 0)=q_{l}(z, 0)$, and applying (197) to $\left\langle(1-\mathbb{P}) a(x, t) c(z, 0), q_{i}\right\rangle=$ $\int \mathrm{d} y\left\langle(1-\mathbb{P}) a(x, t) c(z, 0) q_{i}(y, 0)\right\rangle^{\text {eul }}$, we find

$$
\left\langle\left(1-\mathbb{P}_{\tau}\right) a(x, t) q_{k}(y, 0) q_{l}(z, 0)\right\rangle^{\mathrm{eul}}=\left\langle\left(1-\mathbb{P}_{\tau}\right) a(x, t), q_{i}, q_{j}\right\rangle \mathrm{S}_{k}^{i}(x-y, t) \mathrm{S}_{l}^{j}(x-z, t) .
$$

This shows (189). Finally, writing the usual hydrodynamic projection formula $\left\langle q_{j}(x, t)\right.$ $b(y, 0)\rangle^{\text {eul }}=\mathrm{S}_{j k}(x-y, t) \mathrm{C}^{k l}\left\langle q_{l}, b\right\rangle$ and applying (197) to (196), we obtain

$$
\begin{aligned}
\left\langle\left(1-\mathbb{P}_{\tau}\right) a(x, t) b(y, 0) c(z, 0)\right\rangle^{\mathrm{eul}} & =\left\langle\left(1-\mathbb{P}_{\tau}\right) a(x, t) c(z, 0) q_{i}(y, 0)\right\rangle C^{i j}\left\langle q_{j}, b\right\rangle \\
& =\left\langle\left(1-\mathbb{P}_{\tau}\right) a(x, t) \mathbb{P} \mathcal{b}(y, 0) c(z, 0)\right\rangle .
\end{aligned}
$$

Applying the same formula for $c$ instead of $b$, we obtain (187).

\section{Appendix 6: Representation of Bilinear Charges}

Using the matrix notation, let us introduce the projected three-point coupling symmetric matrix (see (190))

$$
\mathrm{M}_{i j}^{a}=\left\langle\left(1-\mathbb{P}_{\tau}\right) a, q_{i}, q_{j}\right\rangle
$$

as well as the Euler-scale correlation matrix

$$
\mathrm{E}_{i j}^{a}(x, y, t)=\left\langle\left(1-\mathbb{P}_{\tau}\right) a(0,0) q_{i}(x, t) q_{j}(y, t)\right\rangle^{\mathrm{eul}} .
$$

Then, from (189),

$$
\mathrm{E}^{a}(x, y, t)=\mathrm{S}^{\mathrm{T}}(x, t) \mathrm{M}^{a} \mathrm{~S}(y, t)
$$


where we used space-time reversal symmetry. Diagonalising the flux Jacobian as (169) we get

$$
\begin{aligned}
\mathrm{E}^{a}(x, y, t) & =\mathrm{S}^{\mathrm{T}}(x, t) \mathrm{M}^{a} \mathrm{~S}(y, t) \\
& =\mathrm{R}^{-1} \delta\left(x-v^{\text {eff }} t\right) \mathrm{RM}^{a} \mathrm{R}^{\mathrm{T}} \delta\left(y-v^{\text {eff }} t\right) \mathrm{R}^{-\mathrm{T}}
\end{aligned}
$$

Since $\int_{\mathbb{R}} \mathrm{d} x \mathrm{~S}(x, t)=\mathbf{1}$, we find from (202) that

$$
Q_{i j ; \mathscr{H}^{\prime \prime}}(a)=\int \mathrm{d} x \mathrm{~d} y \mathrm{E}_{i j}^{a}(x, y, t)=\mathrm{M}_{i j}^{a}
$$

in agreement with the definition. Further, it is clear that

$$
\lim _{t \rightarrow \infty} \int_{\mathbb{R}} \mathrm{d} x \int_{x-L}^{x+L} \mathrm{~d} y E^{a}(x, y, t)=0
$$

in agreement with the usual two-point hydrodynamic projections.

Most importantly, it is simple to verify that

$$
\int_{\mathbb{R}} \mathrm{d} t \int_{\mathbb{R}} \mathrm{d} x \int_{x-L}^{x+L} \mathrm{~d} y\left[\operatorname{RE}^{a}(x, y, t) \mathrm{R}^{\mathrm{T}}\right]_{i j}=\frac{2 L}{\left|v_{i}^{\text {eff }}-v_{j}^{\text {eff }}\right|}\left[\mathrm{RM}^{a} \mathrm{R}^{\mathrm{T}}\right]_{i j} .
$$

Therefore, defining the observable

$$
w_{k l}=\frac{1}{2 L} \int_{-L}^{L} \mathrm{~d} x\left[\mathrm{R}^{-1}\right]_{k}^{i}(\mathrm{R} q)_{i} \iota_{x}(\mathrm{R} q)_{j}\left|v_{i}^{\text {eff }}-v_{j}^{\text {eff }}\right| \mathrm{R}_{l}^{j}
$$

we see that

$$
\left(w_{k l}, a\right)_{\mathcal{H}^{\prime \prime}}=\int \mathrm{d} t\left\langle\tau_{t} w_{k l},(1-\mathbb{P}) a\right\rangle=\mathrm{M}_{k l}^{a}=Q_{k l ; \mathcal{H}^{\prime \prime}}(a) .
$$

Thus we have found the element in $\mathscr{H}^{\prime \prime}$ representing the quadratic charges as per (143).

Naturally, we can consider the linear normal modes

$$
q_{I}=(\mathrm{R} q)_{I}
$$

and in this basis

$$
w_{I J}=\frac{1}{2 L} \int_{-L}^{L} \mathrm{~d} x q_{I} \iota_{x} q_{J}\left|v_{I}^{\mathrm{eff}}-v_{J}^{\mathrm{eff}}\right|
$$

and

$$
\left(w_{I J}, a\right)_{\mathscr{H}^{\prime \prime}}=Q_{I J ; \mathscr{H}^{\prime \prime}}(a) .
$$

\section{Appendix 7: Prosen's Quadratically Extensive Charges}

For definiteness I take again the situation of Sect. 3.2, with $\iota_{x}$ and $\tau_{t}$ the space and time translations; however the construction is rather general.

Prosen introduced in [10] charges which grow quadratically with the length of the region on which they are supported, and showed that they led to a lower bound for diffusion. The quadratically extensive charges defined in Sect. 4.2 are not exactly of the same type. Here I discuss Prosen's construction, but now interpreted via the Hilbert space structures I have introduced. I reproduce a diffusion bound that is slightly stronger than that obtained in [10], and that agrees with the bounds one can obtain from the charges of Sect. 4.2. 
Here, the condition of projection at infinity (or clustering) (34), used sequentially for $\mathcal{H} \stackrel{\iota}{\rightarrow} \mathcal{H}^{\prime}$, and then for $\mathcal{H}^{\prime} \stackrel{\iota}{\rightarrow} \mathcal{H}^{\prime \prime}$, as it is in Sect. 4.2, is not quite strong enough. Instead, a stronger condition is needed: a projection at infinity that is uniform with respect to both $\iota_{x}$ and $\tau_{t}$

$\left\langle\left(1-\mathbb{P}_{\tau}\right)\left(1-\mathbb{P}_{\iota}\right) a(x, t), b\right\rangle_{\mathcal{H}} \rightarrow 0$ sufficiently fast and uniformly as $|x|,|t| \rightarrow \infty \quad(a, b \in \mathcal{V})$.

Of course, in my original construction, $\mathbb{P}_{\iota}$ acts on $\mathcal{H}$, but $\mathbb{P}_{\tau}$ acts on $\mathcal{H}^{\prime}$. Here and below, however, we need to consider both acting on $\mathcal{H}$. For this purpose, we select a representative in $\mathscr{H}$ of the $\mathscr{H}^{\prime}$-equivalence class for each conserved density $q_{i}$ (as discussed in Remark 3.5 and Sect. 5), and apply the basic formula (50). The results below are independent of the choice of representative. By the usual hydrodynamic projection arguments, projecting out the conserved quantities as in (212) indeed should make the correlator vanish in all of space-time fast enough.

Consider a sequence $Q=\left(Q_{n} \in \mathcal{V}: n \in \mathbb{N}\right)$ of elements of $\mathcal{V} \subset \mathcal{H}$. Assume, without loss of generality, that $Q_{n}=\left(1-\mathbb{P}_{\tau}\right)\left(1-\mathbb{P}_{\iota}\right) Q_{n}$.

Suppose the sequence satisfies the following conditions:

$1^{\prime \prime}$. There exists $\gamma>0$ such that $\left\|Q_{n}\right\|_{\mathscr{H}}^{2}<\gamma n^{2}$ for all $n \in \mathbb{N}$.

$2^{\prime \prime}$. The limit $Q_{\mathscr{H}^{\prime \prime}}(a):=\lim _{n \rightarrow \infty}\left\langle Q_{n}, a\right\rangle_{\mathcal{H}}$ exists for all $a \in \mathcal{V}$.

$3^{\prime \prime}$. There exists $k>0$ and $v_{+}>v_{-} \in \mathbb{R}$ such that $\lim _{n \rightarrow \infty} \sup _{(x, t),(y, s) \in D_{n, k, v_{-}, v_{+}} \mid}$ $\left\langle Q_{n}, a(x, t)-a(y, s)\right\rangle_{\mathcal{H}} \mid=0$ for all $a \in \mathcal{V}$, where

$$
D_{n, k, v_{-}, v_{+}}=\left\{(x, t):-k n+v_{+} t<x<k n+v_{-} t,|t|<\frac{2 k n}{v_{+}-v_{-}}\right\} .
$$

Condition $1^{\prime \prime}$ is the quadratic extensivity. Condition $2^{\prime \prime}$ is the usual requirement of the existence of the limit action. Condition $3^{\prime \prime}$ is the requirement of space-time translation invariance. The special (possibly slanted) diamond shape of the domain $D_{n, k, v_{-}, v_{+}}$is from the intuition that $Q_{n}$ is essentially homogeneous and time-independent on an interval [ $\left.-k n, k n\right]$, but under evolution, the effect of its boundary points moves within the interval; the left (right) boundary point move at velocity $v_{+}\left(v_{-}\right)$. This is the picture of quadratically extensive "almost" conserved charges considered by Prosen (see [10, Fig. 1]). The terminology "almost" was used because of the motion of the boundary points. However this motion is generic, and the result (the limit action of Point 2') is truly time-translation invariant; hence I find there is no need for the adverb "almost".

In quantum spin chains, or whenever a Lieb-Robinson bound exists, typically one can take any

$$
v_{+}=-v_{-}>v_{\mathrm{LR}}, \quad v_{\mathrm{LR}} \text { the Lieb-Robinson velocity. }
$$

$v_{\text {LR }}$ represents the maximal velocity of propagation of local disturbances as set by the dynamics. The Lieb-Robinson velocity is not state dependent, but for a given state it is generically an over-estimate of the actual propagation velocities. It is natural to assume that it is the spectrum of the flux Jacobian (see Appendix 2) that describes all relevant velocities, much as I did in Sect. 5.3, although this is hard to make rigorous for any given sequence $Q$. Under this hydrodynamic picture, one may take any

$$
v_{-}<\inf \left(v^{\text {eff }}\right), \quad v_{+}>\sup \left(v^{\text {eff }}\right) \quad \text { (hydrodynamic picture), }
$$

and this ultimately gives stronger bounds. 
Note that with the uniform clustering condition (212), the inner product (41) on $\mathscr{H}^{\prime \prime}$ can be written as

$$
\langle a, b\rangle_{\mathscr{H}^{\prime \prime}}=\lim _{n \rightarrow \infty} \int_{n D} \mathrm{~d} x \mathrm{~d} t\left\langle\left(1-\mathbb{P}_{\tau}\right)\left(1-\mathbb{P}_{\iota}\right) a(x, t), b\right\rangle_{\mathcal{H}}
$$

for any bounded open domain $D \in \mathbb{R}^{2}$ containing 0 , and a formula similar to (37) holds:

$$
\langle a, b\rangle_{\mathcal{H}^{\prime \prime}}=\lim _{n \rightarrow \infty} \frac{1}{|n D|} \int_{n D} \mathrm{~d} x \mathrm{~d} t \int_{n D} \mathrm{~d} y \mathrm{~d} s\left\langle\left(1-\mathbb{P}_{\tau}\right)\left(1-\mathbb{P}_{\iota}\right) a(x, t), \mathcal{b}(y, s)\right\rangle_{\mathcal{H}} .
$$

We need to prove that $Q_{\mathcal{H}^{\prime \prime}}$ is bounded with respect to $\mathcal{H}^{\prime \prime}$. The proof of boundedness is similar to the case of linearly extensive charges. By Point $3^{\prime \prime}$, there exists $k>0, v_{+}>v_{-} \in \mathbb{R}$, and $\delta_{n}>0$ with $\lim _{n \rightarrow \infty} \delta_{n}=0$, such that

$$
\left\langle Q_{n}, a\right\rangle_{\mathscr{H}}=\frac{v_{+}-v_{-}}{4 k^{2} n^{2}} \int_{D_{n, k, v}} \mathrm{~d} x \mathrm{~d} t\left\langle Q_{n},\left(1-\mathbb{P}_{\tau}\right)\left(1-\mathbb{P}_{\iota}\right) a(x, t)\right\rangle_{\mathcal{H}}+\delta_{n}
$$

where I used $\left|D_{n, k, v_{-}, v_{+}}\right|=4 k^{2} n^{2} /\left(v_{+}-v_{-}\right)$. The first term on the right-hand side is bounded by using the Cauchy-Schwartz inequality:

$$
\leq \frac{\sqrt{v_{+}-v_{-}}}{2 k n}\left\|Q_{n}\right\|_{\mathscr{H}}\left[\frac{v_{+}-v_{-}}{4 k^{2} n^{2}} \int_{D} \mathrm{~d} x \mathrm{~d} t \int_{D} \mathrm{~d} y \mathrm{~d} s\left\langle\left(1-\mathbb{P}_{\tau}\right)\left(1-\mathbb{P}_{\iota}\right) a(x, t), a(y, s)\right\rangle_{\mathscr{H}}\right]^{1 / 2}
$$

where $D=D_{n, k, v_{-}, v_{+}}$. By using (217), we obtain

$$
Q_{\mathscr{H}^{\prime \prime}}(a) \leq \frac{\sqrt{\left(v_{+}-v_{-}\right) \gamma}}{2 k}\|a\|_{\mathscr{H}^{\prime \prime}} .
$$

As a consequence, for every $Q_{\mathscr{H}^{\prime \prime}}$, there exists $b \in \mathscr{H}^{\prime \prime}$ such that

$$
Q_{\mathcal{H}^{\prime \prime}}(a)=\langle\mathcal{b}, a\rangle_{\mathcal{H}^{\prime \prime}} \quad \forall a \in \mathcal{V}, \quad\|\mathcal{b}\|_{\mathscr{H}^{\prime \prime}} \leq \frac{\sqrt{\left(v_{+}-v_{-}\right) \gamma}}{2 k} .
$$

Using known space-time uniformity of correlation functions in order to establish Point $3^{\prime \prime}$, and in particular quantifying the clustering property (212), the result (221) and (220), with (214), can be expressed in a quite rigorous fashion in quantum spin chains; see the arguments in [10].

Therefore we obtain a statement that parallels (89),

$$
\mathfrak{L}_{a, a} \geq \frac{4 k^{2}}{\left(v_{+}-v_{-}\right) \gamma}\left|Q_{\mathcal{H}^{\prime \prime}}(a)\right|^{2} .
$$

With a Lieb-Robinson bound, (214), in the second line, $v_{+}=-v_{-}=v_{\mathrm{LR}}$ (taking the limit over all bounds for $\mathfrak{L}_{a, a}$ ). With the hydrodynamic picture (215), in the second line we can instead set $v_{-}=\inf \left(v^{\text {eff }}\right), v_{+}=\sup \left(v^{\text {eff }}\right)$.

Let me make the connection with Prosen's bound (obtained in the context of quantum chains) more explicit. Following Prosen, I assume that there is no ballistic transport, hence $\mathbb{P}_{\tau}=0$, which simplifies the discussion. Prosen requires a sequence $\left(Q_{n}\right)$ such that $\lim _{n \rightarrow \infty}\left\langle Q_{n} a\right\rangle^{\mathrm{c}}$ exists, which is quadratically growing, $\left\langle Q_{n}^{2}\right\rangle^{\mathrm{c}} \leq \gamma n^{2}$ for some $\gamma>0$. Without loss of generality, $Q_{n}$ is hermitian, and $n$ represents the length of the interval on which $Q_{n}$ is supported. Prosen assumes $Q_{n}$ to be almost homogeneous (homogeneous except near the boundaries of the support), and almost conserved (the time derivative only involves terms at the boundaries of the support). These requirements can be seen to satisfy the three points $1^{\prime}$, 
$2^{\prime}$ and $3^{\prime}$ above. With strong enough clustering (for instance, exponential) and $v_{+}>v_{-} \in \mathbb{R}$ the maximal and minimal propagation velocities, then in (222) we take $k=1 / 2$, and we have

$$
\mathfrak{L}_{a, a} \geq \lim _{n \rightarrow \infty} \frac{\left|\left\langle Q_{n} a\right\rangle^{\mathrm{c}}\right|^{2}}{\left(v_{+}-v_{-}\right) \gamma} .
$$

where I use the explicit form $Q_{\mathscr{H}^{\prime \prime}}(a)=\lim _{n \rightarrow \infty}\left\langle Q_{n} a\right\rangle^{\mathrm{c}}$.

This is an improvement on the bound found in [10] in two ways. First, with the LiebRobinson bound, the denominator is $2 v_{\mathrm{LR}} \gamma$. This is a stronger bound than that with factor 8 instead of 2 found in [10]. The difference can be traced back to the use of a reduced volume of integration in [10], instead of the full diamond $D_{n, k, v_{-}, v_{+}}$. Second, the hydrodynamic velocities are bounded by the Lieb-Robinson velocity $\left|v_{+}\right|<v_{\mathrm{LR}}$ and $\left|v_{-}\right|<v_{\mathrm{LR}}$. Thus, within the hydrodynamic picture, the result is stronger.

\section{Appendix 8: Superdiffusion Bound from Prosen-Type Charges}

I show that one may obtain the superdiffusion bound os Sect. 5.4 by using instead the Prosentype charges of Appendix 7.

The form (177) also suggests that we define an $\alpha^{\prime}$-sequence $\left(Q_{n} \in \mathcal{V}\right)$, for $\alpha^{\prime}>0$, as one that satisfies Points $1^{\prime \prime}$ and $2^{\prime \prime}$ of Appendix 7, as well as the following space-time invariance condition:

$3^{\prime \prime \prime}$. There exists $k>0$ and $\lambda>0$ such that $\lim _{n \rightarrow \infty} \sup _{(x, t),(y, s) \in D_{n, k, \lambda}} \mid\left\langle Q_{n}, a(x, t)-\right.$ $a(y, s)\rangle_{\mathcal{H}} \mid=0$ for all $a \in \mathcal{V}$, where

$$
D_{n, k, \lambda}=\left\{(x, t):-k n+|\lambda t|^{\alpha^{\prime}}<x<k n-|\lambda t|^{\alpha^{\prime}},|\lambda t|<(k n)^{1 / \alpha^{\prime}}\right\} .
$$

The region $D_{n, k, \lambda}$ is an elongated, curvy diamond, whose sides are power-law curves with power $\alpha^{\prime}$. This is simply the region where we would expect space-time invariance for the Prosen-type charge generated by the sequence

$$
Q_{I I ; n}=\left(1-\mathbb{P}_{\tau}\right)\left(1-\mathbb{P}_{\iota}\right)\left[\int_{-n}^{n} \mathrm{~d} x q_{I}(x)\right]^{2} .
$$

Indeed, its boundaries should move at the velocity $v_{I}^{\text {eff }}=0$ (assumed to vanish for simplicity) but extend superdiffusively, and uniformity in space-time is only expected away from this extension. Hence, the sequence $Q_{n}=Q_{I I ; n}$ chosen as (225) is expected to be an $\alpha^{\prime}$-sequence for all $\alpha^{\prime}>\alpha$. Naturally, with this sequence, the result is

$$
\lim _{n \rightarrow \infty}\left\langle Q_{I I ; n}, a\right\rangle_{\mathcal{H}}=Q_{I I ; \mathscr{H}^{\prime \prime}}(a)=\left\langle Q_{I}^{2} a\right\rangle^{\mathrm{c}} .
$$

Note that the volume of the region is

$$
\left|D_{n, k, \lambda}\right|=\frac{4 \alpha^{\prime}}{\left(\alpha^{\prime}+1\right) \lambda}(k n)^{1+1 / \alpha^{\prime}} .
$$

An analysis as in (218) and (219) gives

$$
\left|\left\langle Q_{n}, a\right\rangle_{\mathscr{H}}\right| \leq \sqrt{\frac{\left\|Q_{n}\right\|_{\mathscr{H}}^{2}}{n^{2}}}\left[\frac{n^{2}}{\left|D_{n, k, \lambda}\right|^{2}} \int_{D_{n, k, \lambda}} \mathrm{d} x \mathrm{~d} t \int_{D_{n, k, \lambda}} \mathrm{d} y \mathrm{~d} s\left\langle\left(1-\mathbb{P}_{\tau}\right)\left(1-\mathbb{P}_{\iota}\right) a(x, t), a(y, s)\right\rangle_{\mathcal{H}}\right]^{1 / 2} .
$$


Let us apply this on $j_{I}$. Consider (184), and the fact that, thanks to (177), the two-point function $\left\langle\left(1-\mathbb{P}_{\tau}\right)\left(1-\mathbb{P}_{\iota}\right) j_{I}(x, t), j_{I}(0,0)\right\rangle_{\mathcal{H}}$ is supported along a ballistic trajectory of velocity $v_{I}^{\text {eff }}=0$, as per (180). Thus, at large $n$, we have

$$
\begin{aligned}
& \int_{D_{n, k, \lambda}} \mathrm{d} x \mathrm{~d} t \int_{D_{n, k, \lambda}} \mathrm{d} y \mathrm{~d} s\left\langle\left(1-\mathbb{P}_{\tau}\right)\left(1-\mathbb{P}_{\iota}\right) j_{I}(x, t), j_{I}(y, s)\right\rangle_{\mathcal{H}} \\
& \quad \sim c \int_{|s|<\frac{(k n)^{1 / \alpha^{\prime}}}{\lambda}} \mathrm{d} s \int_{|y|<k n-(\lambda s)^{\alpha^{\prime}}} \mathrm{d} y \int_{|t|<\frac{(k n-y)^{1 / \alpha^{\prime}}}{\lambda} \mathrm{d} t|t-s|^{2 \alpha-2} \quad(n \rightarrow \infty)}
\end{aligned}
$$

where $c=\alpha(2 \alpha-1) \lambda^{2 \alpha} \int \mathrm{d} u u^{2} f(u)$. A simple scaling analysis of this integral then gives

$$
\int_{D_{n, k, \lambda}} \mathrm{d} x \mathrm{~d} t \int_{D_{n, k, \lambda}} \mathrm{d} y \mathrm{~d} s\left\langle\left(1-\mathbb{P}_{\tau}\right)\left(1-\mathbb{P}_{\iota}\right) j_{I}(x, t), j_{I}(y, s)\right\rangle_{\mathcal{H}} \sim c n^{1+2 \alpha / \alpha^{\prime}} \quad(n \rightarrow \infty) .
$$

Combining this with (227) and (228), we find that if $Q_{I I ; \mathcal{H}^{\prime \prime}}\left(j_{I}\right) \neq 0$, then we must have

$$
1+\frac{2 \alpha-2}{\alpha^{\prime}} \geq 0 \Rightarrow \alpha \geq \frac{2-\alpha^{\prime}}{2} .
$$

This is valid for all $\alpha^{\prime}>\alpha$, and the tightest lower bound is obtained by taking $\alpha^{\prime}$ as near to possible to $\alpha$. Thus we conclude

$$
\alpha \geq \frac{2}{3}
$$

This shows that there is superdiffusion. Furthermore, the lower bound on $\alpha$ is indeed the expected KPZ superdiffusive exponent. Clearly, if we further impose that the bounding argument above, for $\left|Q_{I I ; \mathcal{H}^{\prime \prime}}\left(j_{I}\right)\right|$, gives a finite bound, proportional to the finite norm $\left\|j_{I}\right\|_{\mathcal{H}^{(3-2 \alpha)}}$, then we must have $\alpha=\left(2-\alpha^{\prime}\right) / 2$; but as $\alpha \geq 2 / 3$, we must choose $\alpha^{\prime}=\alpha$ and we obtain $\alpha=2 / 3$. The consideration $\alpha^{\prime}=\alpha$ is more subtle however; Point $3^{\prime \prime \prime}$ must be modified to account for finite corrections. These corrections can be argued to scale appropriately under integration, and the bounding argument can be modified accordingly. A precise analysis may produce bounds on $\lambda$; however this is beyond the scope of this paper.

\section{References}

1. Spohn, H.: Large Scale Dynamics of Interacting Particles. Springer, Heidelberg (1991)

2. Spohn, H.: Nonlinear Fluctuating hydrodynamics for anharmonic chains. J. Stat. Phys. 155, 1191 (2014)

3. Mendl, C.B., Spohn, H.: Current fluctuations for anharmonic chains in thermal equilibrium. J. Stat. Mech. Theor. Exp. 2015, P03007 (2015)

4. Kulkarni, M., Huse, D.A., Spohn, H.: Fluctuating hydrodynamics for a discrete Gross-Pitaevskii equation: mapping to Kardar-Parisi-Zhang universality class. Phys. Rev. A 92, 043612 (2015)

5. Popkov, V., Schadschneider, A., Schmidt, J., Schütz, G.M.: Fibonacci family of dynamical universality classes. PNAS 112, 12645-12650 (2015)

6. Popkov, V., Schadschneider, A., Schmidt, J., Schütz, G.M.: Exact scaling solution of the mode coupling equations for non-linear fluctuating hydrodynamics in one dimension. J. Stat. Mech. 2016, 093211 (2016)

7. Schütz, G.M.: On the Fibonacci Universality classes in nonlinear fluctuating hydrodynamics. In: Gonçalves, O., Soares, A.J. (eds.) From Particle Systems to Partial Differential Equations. Springer Proceedings in Mathematics \& Statistics, p. 149. Springer, New York (2018)

8. Chen, Z., de Gier, J., Hiki, I., Sasamoto, T.: Exact confirmation of 1d nonlinear fluctuating hydrodynamics for a two-species exclusion process. Phys. Rev. Lett. 120, 240601 (2018)

9. Bulchandani, V.B.: Kardar-Parisi-Zhang universality from soft gauge modes. Phys. Rev. B 101, 041411(R) (2020) 
10. Prosen, T.: Lower bounds on high-temperature diffusion constants from quadratically extensive almost conserved operators. Phys. Rev. E 89, 012142 (2014)

11. Bertini, B., Collura, M., De Nardis, J., Fagotti, M.: Transport in out-of-equilibrium $X X Z$ chains: exact profiles of charges and currents. Phys. Rev. Lett. 117, 207201 (2016)

12. Castro-Alvaredo, O.A., Doyon, B., Yoshimura, T.: Emergent hydrodynamics in integrable quantum systems out of equilibrium. Phys. Rev. X 6, 041065 (2016)

13. De Nardis, J., Bernard, D., Doyon, B.: Hydrodynamic diffusion in integrable systems. Phys. Rev. Lett. 121, 160603 (2018)

14. De Nardis, J., Bernard, D., Doyon, B.: Diffusion in generalized hydrodynamics and quasiparticle scattering. Sci. Post Phys. 6, 049 (2019)

15. Gopalakrishnan, S., Huse, D.A., Khemani, V., Vasseur, R.: Hydrodynamics of operator spreading and quasiparticle diffusion in interacting integrable systems. Phys. Rev. B 98, 220303 (2018)

16. Ilievski, E., De Nardis, J., Medenjak, M., Prosen, T.: Super-diffusion in one-dimensional quantum lattice models. Phys. Rev. Lett. 121, 230602 (2018)

17. Ljubotina, M., Znidaric, M., Prosen, T.: Kardar-Parisi-Zhang physics in the quantum Heisenberg magnet. Phys. Rev. Lett. 122, 210602 (2019)

18. Gopalakrishnan, S., Vasseur, R.: Kinetic theory of spin diffusion and superdiffusion in XXZ spin chains. Phys. Rev. Lett. 122, 127202 (2019)

19. Gopalakrishnan, S., Vasseur, R., Ware, B.: Anomalous relaxation and the high-temperature structure factor of XXZ spin chains. PNAS 116, 16250 (2019)

20. De Nardis, J., Medenjak, M., Karrasch, C., Ilievski, E.: Anomalous spin diffusion in one-dimensional antiferromagnets. Phys. Rev. Lett. 123, 186601 (2019)

21. Doyon, B., Spohn, H.: Drude Weight for the Lieb-Liniger Bose Gas. SciPost Phys. 3, 039 (2017)

22. Doyon, B.: Thermalization and pseudolocality in extended quantum systems. Commun. Math. Phys. 351, 155 (2017)

23. Doyon, B.: Hydrodynamic projections and the emergence of linearised Euler equations in one-dimensional isolated systems. arXiv:2011.00611 (2020)

24. Mazur, P.: Non-ergodicity of phase functions in certain systems. Physica 43, 533 (1969)

25. Castella, H., Zotos, X., Prelovšek, P.: Integrability and ideal conductance at finite temperatures. Phys. Rev. Lett. 74, 972 (1995)

26. Zotos, X., Naef, F., Prelovšek, P.: Transport and conservation laws. Phys. Rev. B 55, 11029 (1997)

27. Prosen, T., Ilievski, E.: Families of quasilocal conservation laws and quantum spin transport. Phys. Rev. Lett. 111, 057203 (2013)

28. Ilievski, E., Prosen, T.: Thermodynamic bounds on Drude weights in terms of almost-conserved quantities. Commun. Math. Phys. 318, 809 (2013)

29. Medenjak, M., De Nardis, J., Yoshimura, T.: Diffusion from convection. Sci. Post Phys. 9, 075 (2020)

30. Shin, H.K., Choi, B., Talkner, P., Lee, E.K.: Normal versus anomalous self-diffusion in two-dimensional fluids: memory function approach and generalized asymptotic Einstein relation. J. Chem. Phys. 141, $214112(2014)$

31. Lieb, E.H., Robinson, D.W.: The finite group velocity of quantum spin systems. Commun. Math. Phys. 28, 251 (1972)

32. Bratteli, O., Robinson, D.W.: Operator Algebras and Quantum Statistical Mechanics, vol. 1. Springer, Berlin (1987)

33. Bratteli, O., Robinson, D.W.: Operator Algebras and Quantum Statistical Mechanics, vol. 2. Springer, Berlin (1987)

34. Ilievski, E., Medenjak, M., Prosen, T., Zadnik, L.: Quasilocal charges in integrable lattice systems. J. Stat. Mech. 2016, 064008 (2016)

35. Rudin, W.: Functional Analysis, International Series in Pure and Applied Mathematics. McGraw-Hill, Singapore (1991)

36. Araki, H.: Gibbs states of a one dimensional quantum lattice. Commun. Math. Phys. 14, 120 (1969)

37. Israel, R.B.: Convexity in the Theory of Lattice Gases. Princeton University Press, Princeton (1979)

38. Durnin, J., Bhaseen, M.J., Doyon, B.: Non-equilibrium dynamics and weakly broken integrability. Phys. Rev. Lett. 127, 130601 (2021)

39. Prosen, T.: Quantum invariants of motion in a generic many-body system. J. Phys. A 31, L645 (1998)

40. Prosen, T.: Ergodic properties of a generic nonintegrable quantum many-body system in the thermodynamic limit. Phys. Rev. E 60, 3949 (1999)

41. Bravyi, S., Hastings, M.B., Verstraete, F.: Lieb-Robinson bounds and the generation of correlations and the topological quantum order. Phys. Rev. Lett. 97, 050401 (2006)

42. Doyon, B.: Exact large-scale correlations in integrable systems out of equilibrium. Sci. Post Phys. 5, 054 (2018) 
43. Spohn, H.: Interacting and noninteracting integrable systems. J. Math. Phys. 59, 091402 (2018)

44. Doyon, B., Myers, J.: Fluctuations in ballistic transport from Euler hydrodynamics. Ann. Henri Poincaré 21, 255 (2019)

45. Fagotti, M.: Locally quasi-stationary states in noninteracting spin chains. Sci. Post Phys. 8, 048 (2020)

46. Smirnov, F.: Form Factors in Completely Integrable Models of Quantum Field Theory, vol. 14. World Scientific, Singapore (1992)

47. Cortés Cubero, A., Panfil, M.: Thermodynamic bootstrap program for integrable QFT's: Form factors and correlation functions at finite energy density, JHEP 104 (2019)

48. El, G.A.: The thermodynamic limit of the Whitham equations. Phys. Lett. A 311, 374 (2003)

49. El, G.A., Kamchatnov, A.: Kinetic equation for a dense soliton gas. Phys. Rev. Lett. 95, 204101 (2005)

50. El, G.A., Kamchatnov, A.M., Pavlov, M.V., Zykov, S.A.: Kinetic equation for a soliton gas and its hydrodynamic reductions. J. Nonlinear Sci. 21, 151 (2011)

51. Bulchandani, V.B.: On classical integrability of the hydrodynamics of quantum integrable systems. J. Phys. A 50, 435203 (2017)

52. Kovtun, P., Son, D.T., Starinets, A.O.: Holography and hydrodynamics: diffusion on stretched horizons. J. High Energy Phys. 0310, 064 (2003)

53. Karevski, D., Schütz, G.M.: Charge-current correlation equalities for quantum systems far from equilibrium. Sci. Post Phys. 6, 068 (2019)

54. Bastianello, A., Doyon, B., Watts, G., Yoshimura, T.: Generalized hydrodynamics of classical integrable field theory: the Sinh-Gordon model. Sci. Post Phys. 4, 45 (2018)

Publisher's Note Springer Nature remains neutral with regard to jurisdictional claims in published maps and institutional affiliations. 